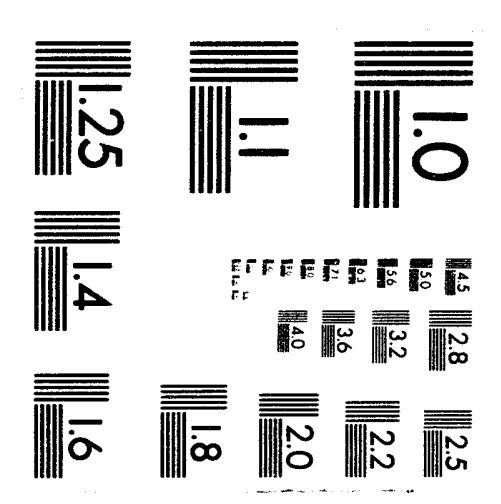



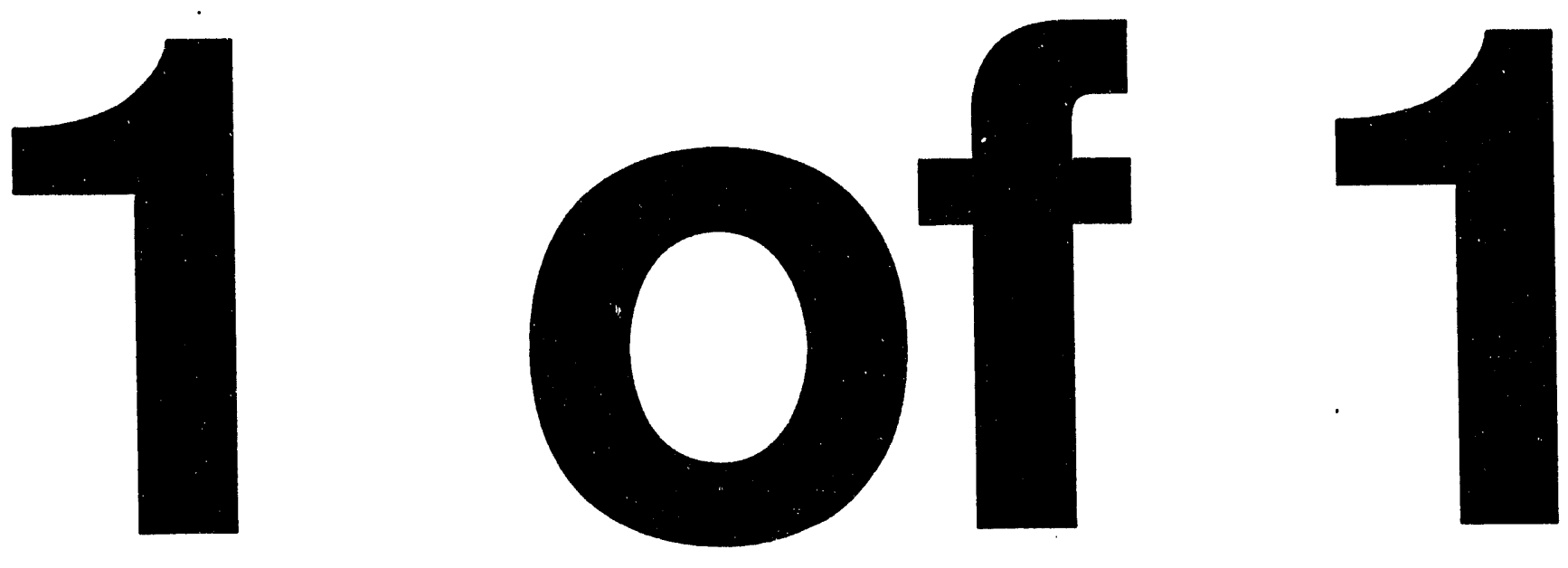


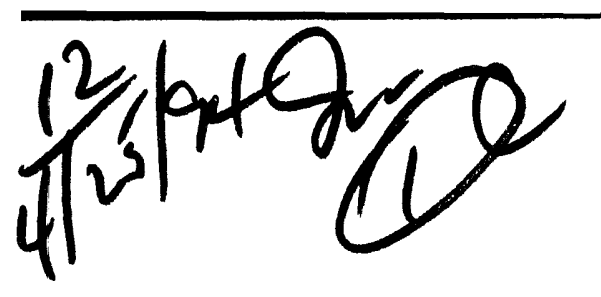

UCRL-ID-116811

\title{
A PHYSICAL OPTICS MODEL FOR SCATTERING BY IRREGULAR TERRAIN AT HF
}

\author{
G. J. Burke
}

March 8, 1994

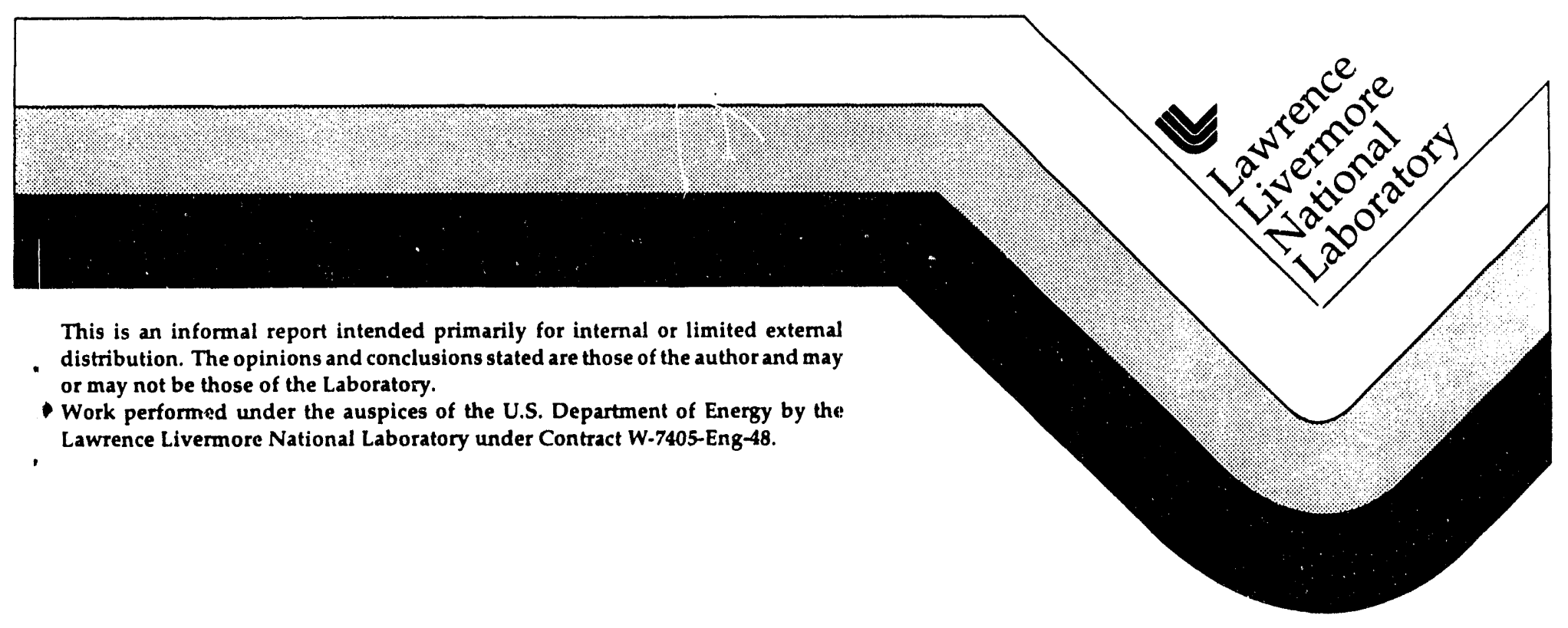




\section{DISCLAIMER}

This document was prepared as an account of work sponsored by an agency of the United States Government. Neither the United States Government nor the University of California nor any of their employees, makes any warranty, express or implied, or assumes any legal liability or responsibility for the accuracy, completeness, or usefulness of an y information, apparatus, produch, or process disclosed, or represents that its use would not infringe privately owned rights. Reference herein to an y specific commercial products, process, or service by trade name, trademark, manufacturer, or otherwise, does not necessarily constitute or imply its endorsement, recommendation, or fa voring by the United States Government or the University of California. The views and opinions of authors expressed herein do not necessarily state or reflect those of the United States Government or the University of California, and shall not be used for advertising or product endorsement purposes.

This report has been reproduced

A vailable to DOE and DOE contractors from the

Crice of Scientific and Technical Information

P.O. Box 62, Oak Ridge, TN 37831

Prices available from (615) 576.8401, FTS 626.8401

A vailable to the public from the

National Technical Information Service

US. Department of Commerce

5285 Port Royal Rd.,

Springfield, VA 22161 


\section{Contents}

Page

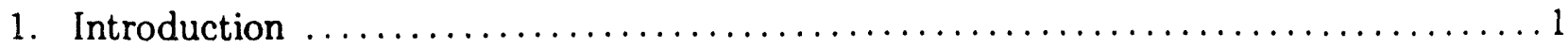

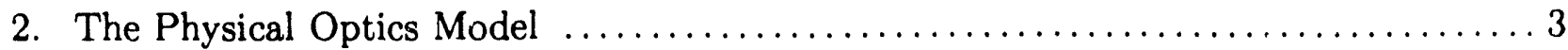

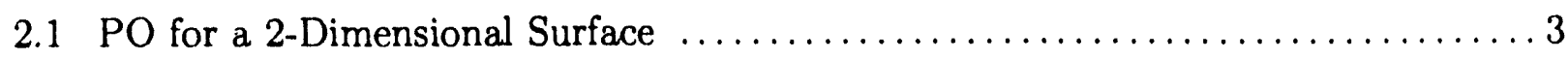

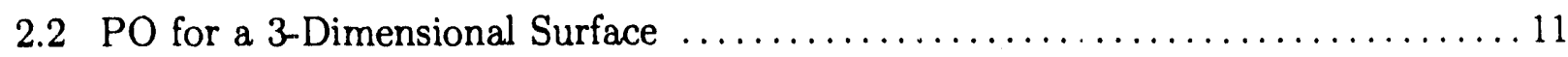

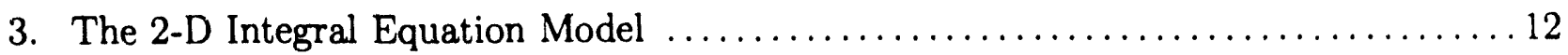

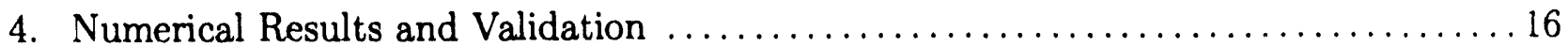

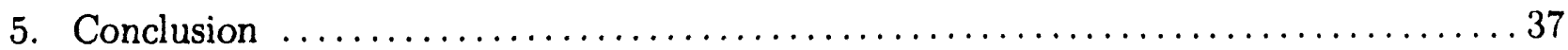

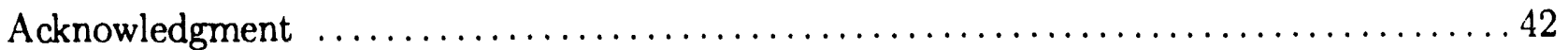

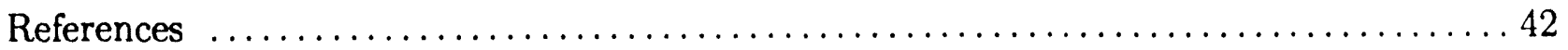

Appendices:

A. Diffraction Coefficients for a Lossy Half-Plane $\ldots \ldots \ldots \ldots \ldots \ldots \ldots \ldots \ldots \ldots$

B. Input for the Physical Optics Codes TPO2D and TPO3D $\ldots \ldots \ldots \ldots \ldots \ldots \ldots$

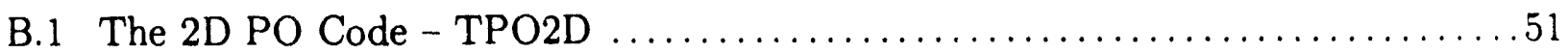

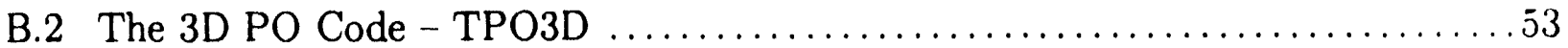

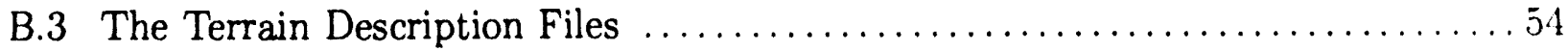

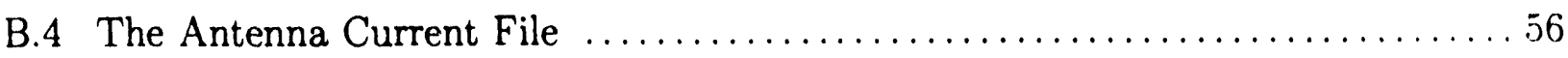




\section{List of Figures}

Fig. No.

Page

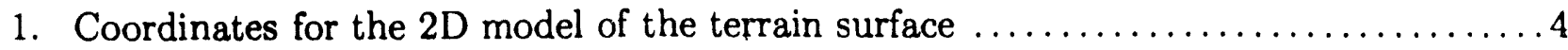

2. Illustration of terms in the PO solution for an antenna near ground $\ldots \ldots \ldots \ldots \ldots 6$

3. Illustration of terms in the PO solution after invoking reciprocity $\ldots \ldots \ldots \ldots \ldots \ldots 8$

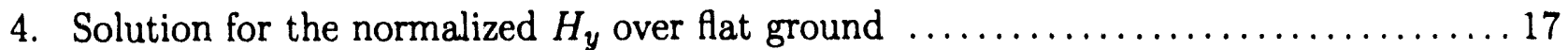

5. Normalized $H_{y}$ over a Gaussian hill from Berry's solution of Hufford's equation .... 17

6. Result from program WAGNER for a Gaussian hill at $1 \mathrm{MHz} \ldots \ldots \ldots \ldots \ldots \ldots 18$

7. Integral equation solution for the Gaussian hill at $1 \mathrm{MHz} \ldots \ldots \ldots \ldots \ldots \ldots \ldots$

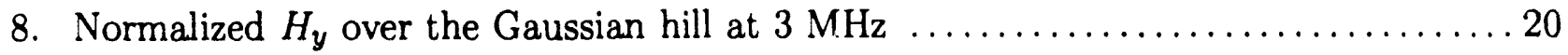

9. Integral equation solution for the Gaussian hill at $10 \mathrm{MHz} \ldots \ldots \ldots \ldots \ldots \ldots \ldots 21$

10. Integral equation solution for the Gaussian hill at $30 \mathrm{MHz} \ldots \ldots \ldots \ldots \ldots \ldots \ldots 22$

11. Radiation patterns of a vertical dipole source $5 \mathrm{~km}$ from a Gaussian hill at $1 \mathrm{MHz} \ldots 23$

12. Radiation patterns of a vertical dipole source $5 \mathrm{~km}$ from a Gaussian hill at $10 \mathrm{MHz} 24$

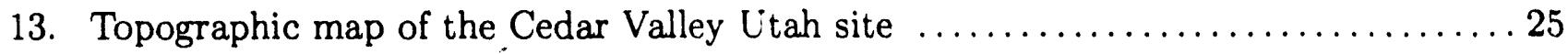

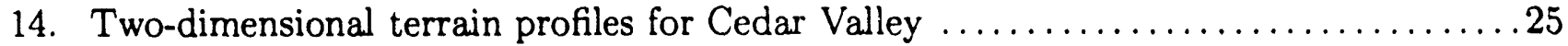

15. Surface plot from interpolation of elevations read from the Cedar Valley map . . ....26 26

16. Normalized field $H_{y}$ over the 2-D Cedar Valley profile at $8.025 \mathrm{MHz} \ldots \ldots \ldots \ldots .27$

17. Normalized field $H_{y}$ over the 2-D Cedar Valley profile at $27.7415 \mathrm{MHz} \ldots \ldots \ldots \ldots 28$

18. Radiation patterns of a vertical dipole in front of the hill at $8.015 \mathrm{MHz} \ldots \ldots \ldots .29$

19. Radiation patterns of a horizontal dipole in front of the hill at $8.015 \mathrm{MHz} \ldots \ldots \ldots 30$

20. Radiation patterns of a vertical dipole on top of the hill at $8.015 \mathrm{MHz} \ldots \ldots \ldots \ldots 31$

21. Radiation patterns of a horizontal dipole in front of the hill at $8.015 \mathrm{MHz} \ldots \ldots \ldots 32$

22. Radiation patterns of a vertical dipole in front of the hill at $27.7415 \mathrm{MHz} \ldots \ldots \ldots 33$

23. Radiation patterns of a horizontal dipole in front of the hill at $27.7415 \mathrm{MHz} \ldots \ldots .34$

24. Radiation patterns of a vertical dipole on top of the hill at $27.7415 \mathrm{MHz} \ldots \ldots \ldots . .35$

25. Radiation patterns of a horizontal dipole in front of the hill at $27.7415 \mathrm{MHz} \ldots \ldots .36$

26. Pattern of a vertical monopole at the West Valley site compared to measurements .. 38

27. Pattern of a horizontal dipole at the West Valley site compared to measurements ... 39

28. Pattern of a vertical monopole at the Hill Top site compared to measurements ..... 40

29. Pattern of a horizontal dipole at the Hill Top site compared to measurements ..... 41

Appendices:

A1. Geometry for diffraction by a half-plane with incidence normal to the edge $\ldots \ldots \ldots 45$

A2. Geometry for diffraction by a half-plane with skew incidence $\ldots \ldots \ldots \ldots \ldots \ldots \ldots 47$ 


\title{
A PHYSICAL OPTICS MODEL FOR SCATTERING BY IRREGULAR TERRAIN AT HF
}

\begin{abstract}
Physical optics models were developed for scattering of HF radiation by irregular terrain when an antenna is located on or near the scattering surface. The primary interest was in skywave radiation patterns for communication links using the ionosphere. Second-order reflections were included in the solution through an image approximation, and the UTD result for an impedance half-plane was used to reduce reflections due to truncating the current distribution on the surface. Arbitrary 2-D or $3-D$ terrain profiles can be entered into the codes, and the source can be an antenna with currents generated by the NEC methodof-moments code or a point source. Results are validated by comparing with 2-D integral equation solutions for actual terrain contours.
\end{abstract}

\section{INTRODUCTION}

Terrain irregularities, such as hills and valleys, can have important effects on the fields radiated by an antenna on the ground. The prediction of these terrain effects is difficult due to the size of the region involved and the variety of conditions that may occur. Also, the terrain scattering depends on the radiation pattern of the antenna, which itself may present a difficult modeling problem. Various methods have been used to model terrain effects, ranging from knife-edge diffraction to more computationally demanding integral equation methods. Much of the work has addressed problems of communication between points on the ground surface, since the ground contour is often a critical factor in these cases. However, terrain scaltering can also have significant effects on the skywave radiation pattern of an antenna. This report describes the development and validation of physical optics (PO) models for scattering of $\mathrm{HF}$ radiation by two- and three-dimensional terrain surfaces, with the primary interest in the skywave radiation pattern at angles used in ionospheric communication links. The source antenna can be a point dipole in 3-D or line source in 2-D or a collection of wire segments and segment currents generated by the NEC antenna modeling program [1].

Several techniques for modeling terrain effects were considered in a previous study [2]. They can be divided into solutions based on integrating induced sources over the ground surface, ray-based methods of geometrical optics or GTD/UTD and differential equation solutions $[3,4]$. In the work reported in [2] a geometrical optics (GO) model was developed for arbitrary 3-D terrain surfaces. The method of Ray Launching [5] was used, in which triangular ray tubes are traced to reflection points on the surface, with the phase, magnitude and ray-tube divergence determined from the surface properties and curvature. Since curved surfaces are modeled, diffraction is not needed to fill in the gaps that would occur in reflections from flat plates. However, GO yields zero field in shadow regions and is limited by caustics in directions where separate reflected rays merge. Geometrical optics can yield accurate results in illuminated regions and allows a relatively fast evaluation of the scattered 
field from a 3-D terrain model. Also, in a related study by Breakall et al. [7] the GTD code $N E C$ - Basic Scattering Code [6] was applied to model scattering from plate approximations of terrain surfaces.

In methods based on integration of surface currents the major effort is usually in determining the induced currents. The most accurate numerical method is the solution of a Fredholm integral equation by the method of moments. The surface usually must be divided into about ten cells per wavelength. If $N_{x}$ cells are used in the $x$ direction and $N_{y}$ in the $y$ direction, a matrix equation of order $N_{x} N_{y}$ must be solved, with solution time proportional to $\left(N_{x} N_{y}\right)^{3}$. Thus, while 2-D models of reasonable size are practical, the matrix solution time limits 3-D models to regions of several wavelengths on a side. The computation time can be greatly reduced by solving a Volterra integral equation derived from the parabolic approximation of the wave equation $[8,9,10]$. Volterra integral equations can be solved by advancing the solution in increments away from the source, with the solution time to the $N$ th point going as $N^{2}$. In addition, particularly with the Volterra equation derived by Ott, the increments on the surface can be much larger than those needed to solve the Fredholm integral equation. The Volterra integral equations are limited to 2-D terrair models and neglect backscattering, but they can yield accurate solutions for many terrain conditions. However, the computation time can become large if the solution is needed over a long distance from the source.

The fastest way of obtaining the surface currents is by the physical optics (PO) solution, which is equivalent to the Kirchhoff approximation in diffraction theory. In PO, the surface currents are determined by the direct incident field from the source, so for $N$ cells the time to evaluate the current is proportional to $N$. As with the integral equation methods the current can be computed once and used to evaluate the scattered field over many angles, while in ray-based methods the entire solution process must be repeated for each radiation angle. However, the time to integrate over the PO currents for many radiation angles can become significant when the region of terrain covers many square wavelengths.

Results of some initial PO calculations for terrain scattering were included in [11]. It has since been found that in order to get accurate results when the antenna is located on the irregular terrain it is necessary to invoke reciprocity to put the source at the distant receiver location and evaluate the field at the original antenna location near ground. In addition, second-order reflections in the vicinity of the antenna and receiver were found to be important. Reflected and diffracted fielc's from the UTD solution for an impedance halfplane were added to the model to reduce ripple in the radiation patterns that would result from truncating the PO currents on the surface. This last extension saves computation time. by reducing the range over which the PO currents must be integrated.

The development of the PO models for 2-D and 3-D terrain scattering is described in Section 2 of this report. In addition, a code for solving the Fredholm integral equations (MFIE for vertical polarization and EFIE for horizontal polarization) was developed to validate the PO models. The solution of these integral equations is described in Section 3. Section 4 contains some results generated to validate the codes. The integral equation solutions are compared with solutions of Volterra integral equations and an analytic result 
for flat ground. Then the radiation patterns obtained by integrating the currents from the integral equations are compared with patterns from the PO solution for a Gaussian hill and an actual ground profile.

\section{THE PHYSICAL OPTICS MODEL}

In PO the current at each point on the surface is determined by the direct, incident field from the source with the assumption that the field reflects as it would from an infinite plane tangent to the surface at that point. Hence PO is sometimes called the tangent-plane approximation. On a lossy surface an impedance boundary condition is enforced and reflected fields are determined by the Fresnel plane-wave reflection coefficients. At points shaded from direct illumination by the source the PO currents are assumed to be zero. PO should yield reasonably accurate currents over illuminated parts of an electrically large surface where the radius of curvature is much greater than the wavelength. The scattered fields obtained by integrating the $\mathrm{PO}$ currents are expected to be most accurate in directions of strongest scattering. In nulls of the total field, and particularly in shadow regions where direct and scattered fields cancel, the accuracy of the $\mathrm{PO}$ result is reduced by the assumption of zero currents on shaded surfaces and the abrupt shadow boundaries. The accuracy of PO for a given application is difficult to predict, and usually must be determined by trial. Hence, results of these models for terrain scattering will be validated by comparison with integral equation solutions.

Both 2-D and 3-D PO models were developed. The 2-D code is faster than 3-D and it is easier to enter the terrain description in 2-D than in 3-D. The 2-D model will often be adequate for determining the radiation pattern in a given vertical plane, but 3-D is needed when important scatterers lie out of the propagation plane.

\subsection{PO for a 2-Dimensional Surface}

For the 2-D PO model the terrain surface will be assumed to vary in height in the $x$ direction as $z=h_{s}(x)$ and be constant in the $y$ direction, with surface normal $\hat{\mathbf{n}}$ and tangent vector $\hat{\mathbf{s}}$ as shown in Figure 1. The lossy ground is characterized by relative permittivity $\epsilon_{r}$ and conductivity $\sigma$, and these parameters may be functions of $x$. Also needed are the wavenumber in air $k_{0}=\omega \sqrt{\mu_{0} \epsilon_{0}}$ and in the earth $k_{g}=k_{0} \sqrt{\tilde{\epsilon}_{g}}$, where $\tilde{\epsilon}_{g}=\epsilon_{r}-j \sigma / \omega \epsilon_{0}$. The wave impedances in air and earth are $\eta_{0}=\sqrt{\mu_{0} / \epsilon_{0}}$ and $\eta_{g}=\eta_{0} / \sqrt{\epsilon_{g}}$, respectively. The time dependence $e^{j \omega t}$ is assumed.

In the PO approximation, the surface fields over illuminated parts of the scattering surface are the sum of the incident and reflected fields with the latter approximated as the incident field multiplied by the Fresnel plane-wave reflection coefficients for incidence on the tangent plane. The reflection coefficient for TE polarization ( $\mathbf{E}$ normal to the plane of incidence) is [12]

$$
R_{\mathrm{TE}}=E_{y}^{r} / E_{y}^{i}=\frac{k_{0} \cos \theta_{i}-k_{g} \cos \theta_{t}}{k_{0} \cos \theta_{i}+k_{g} \cos \theta_{t}}
$$

where $E_{y}^{i}$ and $E_{y}^{r}$ are the $y$ components of incident and reflected electric fields on the surface, 


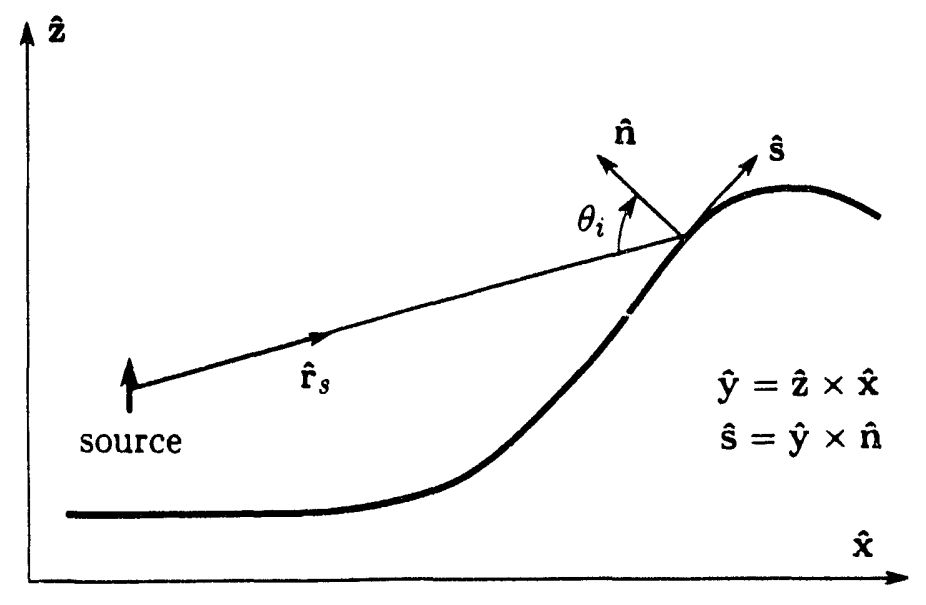

Fig. 1. Coordinates for the 2-D model of the terrain surface.

respectively. For TM polarization

$$
R_{\mathrm{TM}}=H_{y}^{r} / H_{y}^{i}=\frac{k_{g} \cos \theta_{i}-k_{0} \cos \theta_{t}}{k_{g} \cos \theta_{i}+k_{0} \cos \theta_{t}}
$$

The angle $\theta_{i}$ is between the incident ray and the normal, so that $\cos \theta_{i}=-\hat{\mathbf{r}}_{s} \cdot \hat{\mathbf{n}}$, and $\theta_{t}$ is the complex angle of the ray transmitted into the ground

$$
\cos \theta_{t}=\left[1-\left(k_{0} / k_{g}\right)^{2} \sin ^{2} \theta_{i}\right]^{1 / 2}
$$

An arbitrary incident field will be decomposed into TE and TM components, and in 2-D these components are not coupled by scattering. It is possible to solve two scalar scattering problems for the transverse field components, but the vector electric field will be computed since that is the field most often measured. While the measurement points will be in the far field of the antenna, they may not be in the far field of the terrain scattering surface, so $E / H=\eta_{0}$ cannot always be assumed.

If the incident fields at point $\left(x, z_{s}\right)$ on the surface, where $z_{s}=h_{s}(x)$, are $\mathbf{E}^{i}\left(x, z_{s}\right)$ and $\mathbf{H}^{i}\left(x, z_{s}\right)$ the TM components of the total surface fields are

$$
\begin{aligned}
\mathbf{E}_{\mathrm{TM}}^{t}\left(x, z_{s}\right) & =\left(1-R_{\mathrm{TM}}\right)\left[\mathbf{E}^{i}\left(x, z_{s}\right) \cdot \hat{\mathbf{s}}\right] \hat{\mathbf{s}} \\
\mathbf{H}_{\mathrm{TM}}^{t}\left(x, z_{s}\right) & =\left(1+R_{\mathrm{TM}}\right)\left[\mathbf{H}^{i}\left(x, z_{s}\right) \cdot \hat{\mathbf{y}}\right] \hat{\mathbf{y}}
\end{aligned}
$$

and the TE components are

$$
\begin{aligned}
\mathbf{E}_{\mathrm{TE}}^{t}\left(x, z_{s}\right) & =\left(1+R_{\mathrm{TE}}\right)\left[\mathbf{E}^{i}\left(x, z_{s}\right) \cdot \hat{\mathbf{y}}\right] \hat{\mathbf{y}} \\
\mathbf{H}_{\mathrm{TE}}^{t}\left(x, z_{s}\right) & =\left(1-R_{\mathrm{TE}}\right)\left[\mathbf{H}^{i}\left(x, z_{s}\right) \cdot \hat{\mathbf{s}}\right] \hat{\mathbf{s}}
\end{aligned}
$$

The fields from equations (3) through (6) will satisfy the surface-impedance boundary condition $E_{\mathrm{TM}}^{t} /\left(H_{\mathrm{TM}}^{t} \cos \theta_{t}\right)=\cos \theta_{t} E_{\mathrm{TE}}^{t} / H_{\mathrm{TE}}^{t}=\eta_{g}$ if $E^{i} / H^{i}=\eta_{0}$. Applying the equivalence 
principle with fields inside the surface equal to zero yields the following magnetic and electric currents over illuminated parts of the surface

$$
\begin{aligned}
\mathbf{K}_{\mathrm{TM}}(x) & =-\hat{\mathbf{n}} \times \mathbf{E}_{\mathrm{TM}}^{t}\left(x, z_{s}\right)=-\left(1-R_{\mathrm{TM}}\right)\left[\mathbf{E}^{i}\left(x, z_{s}\right) \cdot \hat{\mathbf{s}}\right] \hat{\mathbf{y}} \\
\mathrm{J}_{\mathrm{TM}}(x) & =\hat{\mathbf{n}} \times \mathbf{H}_{\mathrm{TM}}^{t}\left(x, z_{s}\right)=-\left(1+R_{\mathrm{TM}}\right)\left[\mathbf{H}^{i}\left(x, z_{s}\right) \cdot \hat{\mathbf{y}}\right] \hat{\mathbf{s}}
\end{aligned}
$$

and

$$
\begin{aligned}
\mathbf{K}_{\mathrm{TE}}(x) & =-\hat{\mathbf{n}} \times \mathbf{E}_{\mathrm{TE}}^{\ell}\left(x, z_{s}\right)=\left(1+R_{\mathrm{TE}}\right)\left[\mathbf{E}^{i}\left(x, z_{s}\right) \cdot \hat{\mathbf{y}}\right] \hat{\mathbf{s}} \\
\mathbf{J}_{\mathrm{TE}}(x) & =\hat{\mathbf{n}} \times \mathbf{H}_{\mathrm{TE}}^{t}\left(x, z_{s}\right)=\left(1-R_{\mathrm{TE}}\right)\left[\mathbf{H}^{i}\left(x, z_{s}\right) \cdot \hat{\mathbf{s}}\right] \hat{\mathbf{y}}
\end{aligned}
$$

The total surface currents are then

$$
\begin{gathered}
\mathbf{K}(x)=\mathbf{K}_{\mathrm{TE}}(x)+\mathbf{K}_{\mathrm{TM}}(x) \\
\mathrm{J}(x)=\mathbf{J}_{\mathrm{TE}}(x)+\mathbf{J}_{\mathrm{TM}}(x) .
\end{gathered}
$$

At points where the incident field is blocked by other parts of the scattering surface $\mathbf{K}(x)$ and $\mathbf{J}(x)$ are equal to zero in the PO approximation.

The field scattered by the terrain can be obtained by integrating over the surface currents as

$$
\mathbf{E}^{s}(x, z)=-j \omega \mu \int_{x_{1}}^{x_{2}} \overline{\bar{\Gamma}}_{c}\left(\mathbf{r}, \mathbf{r}^{\prime}\right) \cdot \mathbf{J}\left(x^{\prime}\right) d s^{\prime}+\int_{x_{1}}^{x_{2}} \nabla G_{c}\left(\mathbf{r}, \mathbf{r}^{\prime}\right) \times \mathbf{K}\left(x^{\prime}\right) d s^{\prime}
$$

where $d s^{\prime}$ is the element of length on the surface $d s^{\prime}=\sqrt{1+\left[h_{s}^{\prime}\left(x^{\prime}\right)\right]^{2}} d x^{\prime}$ and $\overline{\bar{\Gamma}}_{c}\left(\mathbf{r}, \mathbf{r}^{\prime}\right)$ is the dyadic Green's function. In two dimensions

$$
\begin{aligned}
& \overline{\bar{\Gamma}}_{c}\left(\mathbf{r}, \mathbf{r}^{\prime}\right)=\left(\overline{\overline{\mathbf{I}}}+\frac{1}{k^{2}} \nabla \nabla\right) G_{c}\left(\mathbf{r}, \mathbf{r}^{\prime}\right) \\
& G_{c}\left(\mathbf{r}, \mathbf{r}^{\prime}\right)=\frac{-j}{4} H_{0}^{(2)}\left(k\left|\mathbf{r}-\mathbf{r}^{\prime}\right|\right)
\end{aligned}
$$

where $\mathbf{r}=x \hat{\mathbf{x}}+z \hat{\mathbf{z}}$ is the vector to the field evaluation point, $\mathbf{r}^{\prime}=x^{\prime} \hat{\mathbf{x}}+h_{9}\left(x^{\prime}\right) \hat{\mathbf{z}}$ is the vector to the source point on the surface and $H_{0}^{(2)}$ is the Hankel function. The limits $x_{1}$ and $x_{2}$ should be positive and negative infinity, but in practice the integral is truncated at points beyond which the contributions are negligible.

The direct application of PO with the antenna near ground and a distant receiver is illustrated in Figure 2a. The total field $\mathrm{E}^{a}$ at the evaluation point $\left(x_{r}, z_{r}\right)$ is the sum of the direct field from the source and the field scattered from the terrain

$$
\mathbf{E}^{a}\left(x_{r}, z_{r}\right)=\mathbf{E}^{s}\left(x_{r}, z_{r}\right)+\mathbf{E}^{i}\left(x_{r}, z_{r}\right)
$$

In initial tests of the PO model the antenna was assumed to be over flat ground at a distance from a hill that was the scatterer. In this case the PO currents are significant over the hill and under the antenna but are small over the flat ground between hill and antenna. If the source point and integration point $\mathbf{r}^{\prime}$ are on the flat surface and the source produces a 
a)

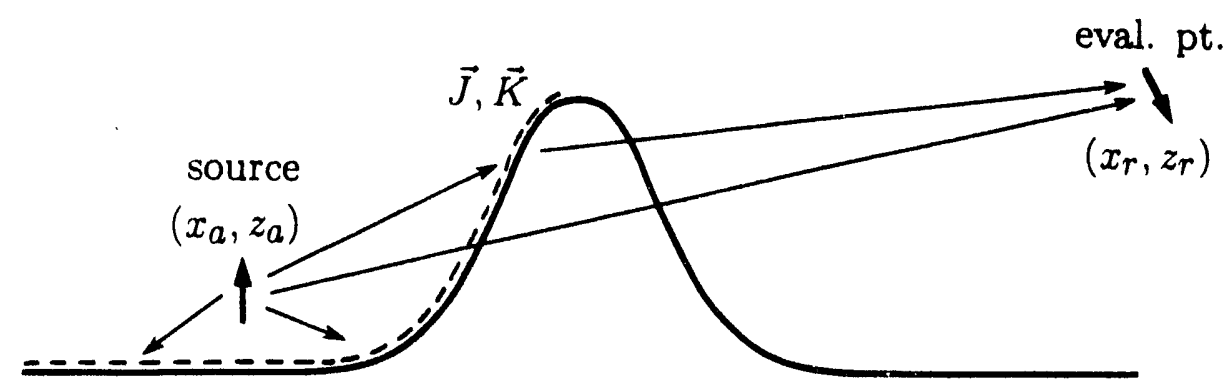

b)

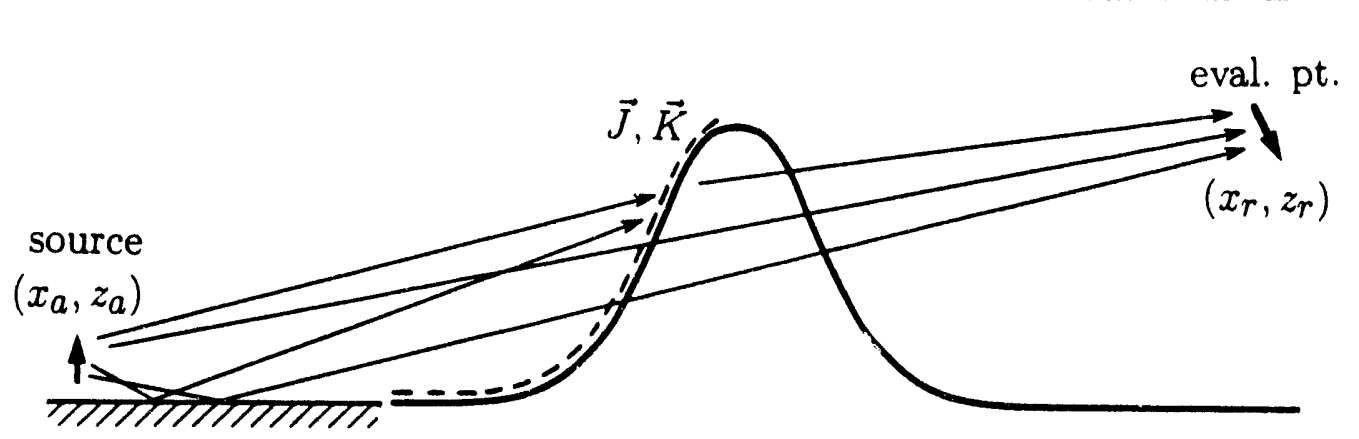

Fig. 2. Illustration of terms in the $P O$ solution for a source at the antenna location near ground, showing: a) First-order PO; b) first-order PO with second-order reflections included at the source.

TEM field, the ŝ components of incident field vanish in equations (7) and (10) while the reflection-coefficient terms vanish in (8) and (9). In this case, it is convenient to stop the PO integral on the flat ground where the contribution is small. The field from the ground under the antenna can then be evaluated by including the field of an image of the antenna multiplied by a reflection coefficient for the ground. If the fields reflected from ground under the antenna are included in the $\mathbf{E}^{i}$ and $\mathbf{H}^{i}$ determining PO currents on the hill, they represent second-order corrections to the first-order PO solution. This situation is illustrated in Figure $2 b$.

This combined geometrical and physical optics model was found to give good results for scattering from a distant hill. The second-order geometrical-optics correction for the source is particularly important for horizontal polarization since the $R_{\mathrm{TE}}$ reflection coefficient is usually larger than $R_{\mathrm{TM}}$. However, this approach could not be extended to cases where the antenna was located in the region of irregular terrain. If the near fields of the antenna are used in evaluating $\mathbf{E}^{i}$ and $\mathbf{H}^{i}$ over the surface, the PO integral in (11) can be evaluated through the region under the antenna to yield an exact scattered field for a ground that is flat and perfectly conducting. However, it is not accurate if the ground is flat and finitely conducting. While the radius of curvature of the ground can be assumed to be much greater than the wavelength, and hence locally flat, a restriction on the ground conductivity is not acceptable. An exact result for flat, finitely conducting ground could be obtained by using the Sommerfeld-integral form for the incident field due to the antenna over ground, but this involves too much complexity and computation time.

To get a result that is accurate for a flat finitely conducting ground, reciprocity was invoked to put the source in the PO solution at the location of the distant receiver and 
evaluate the field at the antenna location near ground. If the original antenna at $\left(x_{a}, z_{a}\right)$ is a current element $I_{a} d \ell$ that produces a field $\mathbf{E}^{a}$ at the receiver location $\left(x_{r}, z_{r}\right)$, then by reciprocity a current element $\mathbf{I}_{r} d \ell$ at $\left(x_{r}, z_{r}\right)$ will produce the field $\mathbf{E}^{r}$ at $\left(x_{a}, z_{a}\right)$ where

$$
\mathrm{E}^{a}\left(x_{r}, z_{r}\right) \cdot \mathbf{I}_{r} d \ell=\mathrm{E}^{r}\left(x_{a}, z_{a}\right) \cdot \mathbf{I}_{a} d \ell .
$$

While FO does not satisfy reciprocity exactly, similar results should be obtained within the accuracy of the solutions. In fact, interchanging source and evaluation points might be expected to make the solution more robust. When the source is near the ground, a small peak in the ground can make a big difference in the extent of the ground that is illuminated, while the distant source will illuminate all of the ground for typical terrain except at low incidence angles. With the source at a large distance, the plane-wave reflection coefficients of (1) and (2) give accurate reflected fields over locally flat ground. Then with the near field used for the scattered field in equation (11), the result is exact for flat finitely conducting ground if the distance to the source is sufficiently large. The components of the PO solution after invoking reciprocity are illustrated in Figure 3a.

With source and evaluation points interchanged it becomes more difficult to include the second-order reflections under the antenna for fields that have already scattered from the terrain. A full second-order PO solution would require the evaluation of $N^{2}$ interaction coefficients for $N$ surface samples, which is the same as for an integral equation solution and too time consuming for large models. A limited second-order PO would require decisions about which interactions to include. It was found that a good approximation of second-order reflections near the antenna could be obtained by evaluating the field at the location of the image of the antenna reflected in the ground. If the field evaluation point (original antenna location) is $\mathbf{r}_{a}=x_{a} \hat{\mathbf{x}}+z_{a} \hat{\mathbf{z}}$ the image location, assuming locally flat ground, is

$$
\mathbf{r}_{a}^{\prime}=x_{a}^{\prime} \hat{\mathbf{x}}+z_{a}^{\prime} \hat{\mathbf{z}}=\mathbf{r}_{a}-2\left[z_{a}-h_{s}\left(x_{a}\right)\right]\left[\hat{\mathbf{z}} \cdot \hat{\mathbf{n}}\left(x_{a}\right)\right] \hat{\mathbf{n}}\left(x_{a}\right) \text {. }
$$

The sum of the direct and reflected fields is then computed as

$$
\mathbf{E}^{r}\left(x_{a}, z_{a}\right)=\mathbf{E}^{i}\left(x_{a}, z_{a}\right)+\mathbf{E}^{s}\left(x_{a}, z_{a}\right)+R_{a}\left[\mathbf{E}^{i}\left(x_{a}^{\prime}, z_{a}^{\prime}\right)+\mathbf{E}^{s}\left(x_{a}^{\prime}, z_{a}^{\prime}\right)\right]
$$

where the two $\mathbf{E}^{s}$ terms come from separate evaluations of (11). To first order, the total field at the image location $\left(x_{a}^{\prime}, z_{a}^{\prime}\right)$ should be zero, since that is the condition used in the equivalence principle to determine the surface currents. The actual field evaluated at the image will approximate the fields that have reflected or diffracted from the surface and then would reflect from the surface again near the antenna. These fields pass through the surface since the first-order PO currents do not know about them. The image will also pick up error fields resulting from the finite integration limits $x_{1}$ and $x_{2}$ or limited integration accuracy, and these errors can be partially cancelled in (12) for horizontal polarization where they are usually most significant.

The fields at the image evaluation point are assumed to actually reflect from the surface to reach the antenna. Hence they should be multiplied by the appropriate reflection coefficients for the ground. For ground with finite conductivity, the reflection coefficient is a function of the angle of incidence $\theta_{i}$, but this angle is not known for the field obtained by 
a)

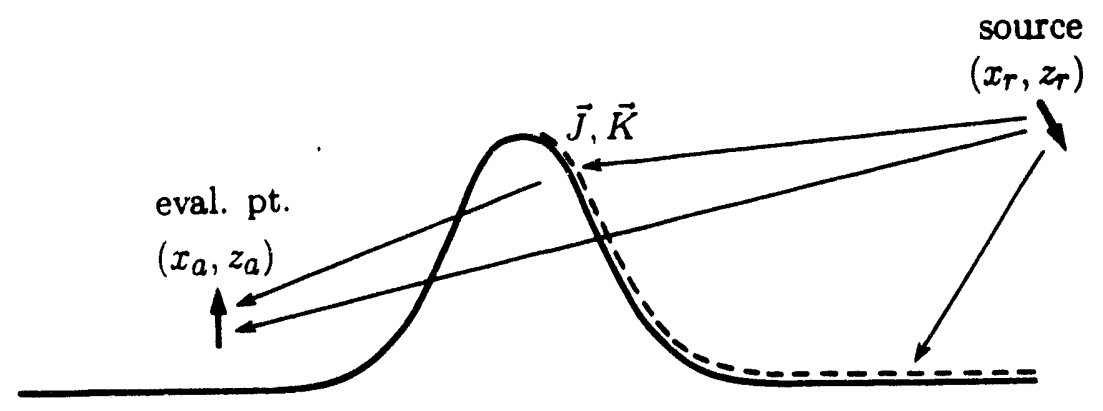

b)

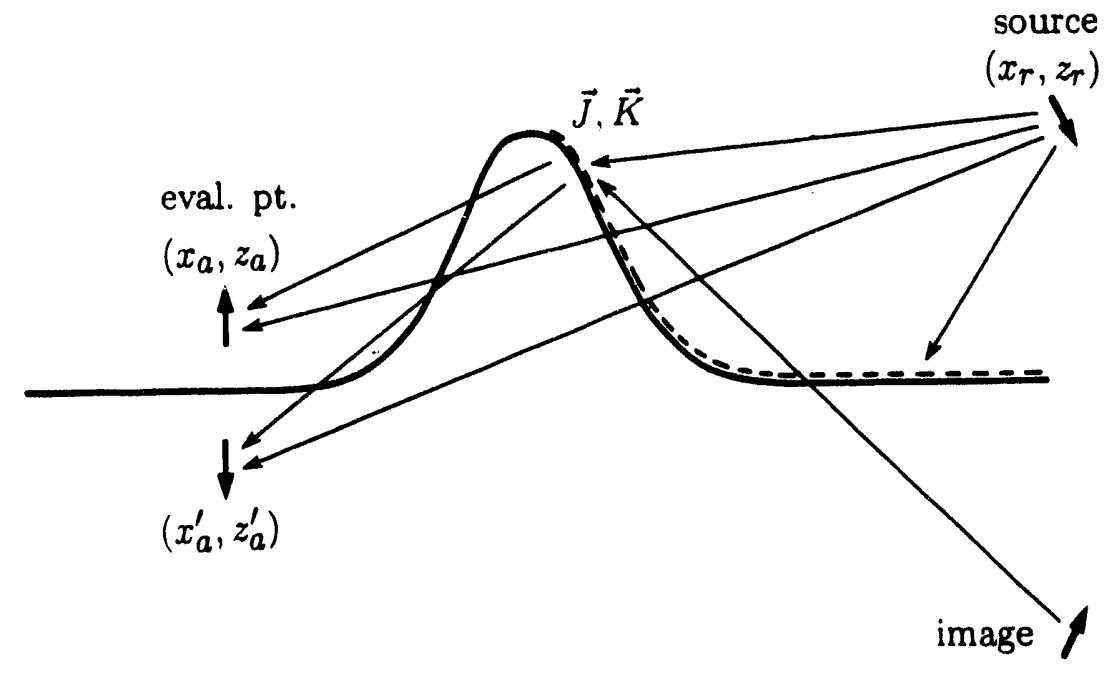

c)

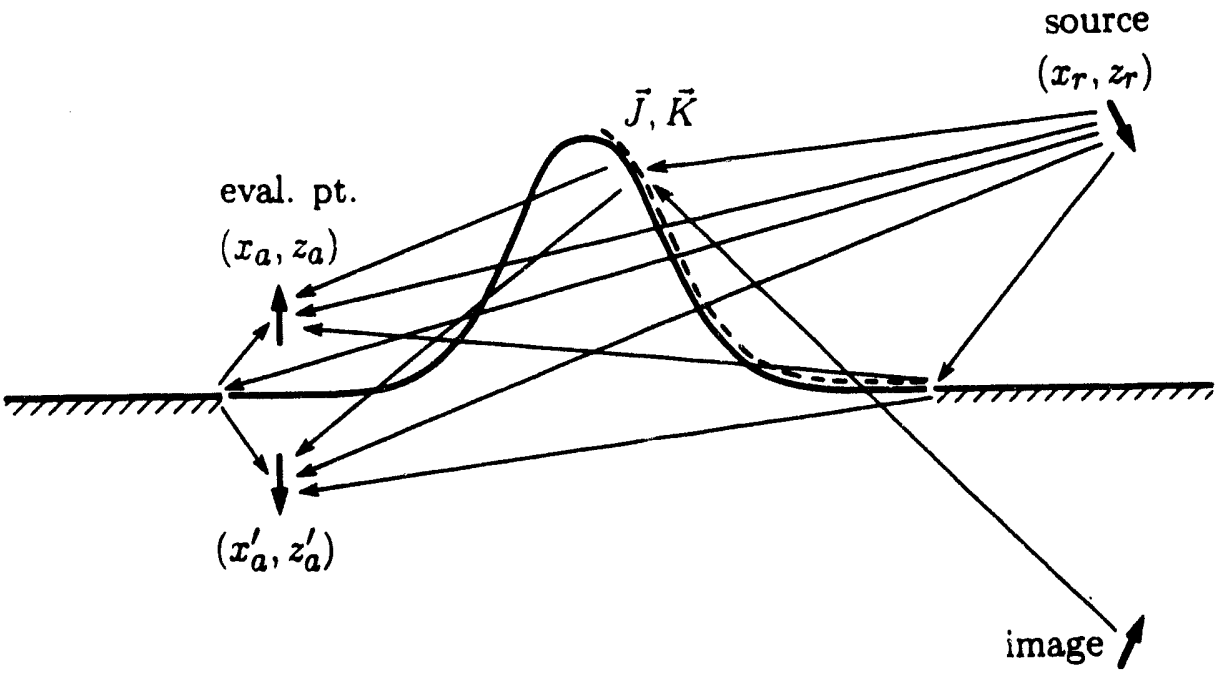

Fig. 3. Illustration of terms in the PO solution after invoking reciprocity to put the source at the location of the distant receiver. a) is simple first-order PO; b) includes second-order image fields for the source and evaluation point; c) adds the reflected and diffracted fields from half-planes extending to infinity.

evaluating the integral in (11). A varying reflection coefficient cannot be included in (11) since that would invalidate the first-order PO solution. Hence an average reflection coeffcient $R_{a}$ was determined by scanning the surface to determine the average angle of a ray from the image at $\left(x_{a}^{\prime}, z_{a}^{\prime}\right)$ to points on the surface that are visible to the image. Fortunately 
the second-order image correction is most important for horizontal polarization where the reflection coefficient is relatively insensitive to incidence angle.

The fields due to the image of the distant source are also added to the direct fields $\mathbf{E}^{i}$ and $\mathbf{H}^{i}$ in (7) through (10) at points where the image illuminates the front side of the surface. For typical terrain, the image of the source illuminates the front of the surface only at low incidence angles. The PO evaluation with second-order corrections to source and receiver is illustrated in Figure $3 \mathrm{~b}$. The direct field from the image of the source to the evaluation point is a first-order term included in the PO evaluation, so it is not included separately.

The final addition to the PO model was to include the reflected and diffracted fields from half-planes extending from the limits of the PO integration at $\left[x_{1}, h_{s}\left(x_{1}\right)\right]$ and $\left[x_{2}, h_{s}\left(x_{2}\right)\right]$ to negative and positive infinity. The UTD result developed by Volakis [13] for a half-plane with impedance boundary condition was used (see Appendix A). In the 2-D model, the relatively simple result in [13] for incidence normal to the edge can be used. When the direct ray from source to evaluation point intercepts the UTD half-plane it is omitted, while in PO it is included but partially cancelled by the PO currents. UTD does not provide a seamless transition from the PO solution due to the different treatments of the edge effects in the two models. However, the inclusion of UTD half-planes allows a large reduction in the range of the $\mathrm{PO}$ integration, and hence reduces the computation time. The total field at the evaluation point is now

$$
\begin{aligned}
\mathbf{E}^{r}\left(x_{a}, z_{a}\right)= & \mathbf{E}^{i}\left(x_{a}, z_{a}\right)+\mathbf{E}^{s}\left(x_{a}, z_{a}\right)+\mathbf{E}_{1}^{\mathrm{HP}}\left(x_{a}, z_{a}\right)+\mathbf{E}_{2}^{\mathrm{HP}}\left(x_{a}, z_{a}\right) \\
& +R_{a}\left[\mathbf{E}^{i}\left(x_{a}^{\prime}, z_{a}^{\prime}\right)+\mathbf{E}^{s}\left(x_{a}^{\prime}, z_{a}^{\prime}\right)+\mathbf{E}_{1}^{\mathrm{HP}}\left(x_{a}^{\prime}, z_{a}^{\prime}\right)+\mathbf{E}_{2}^{\mathrm{HP}}\left(x_{a}^{\prime}, z_{a}^{\prime}\right)\right]
\end{aligned}
$$

where $\mathbf{E}_{1}^{\mathrm{HP}}$ and $\mathbf{E}_{2}^{\mathrm{HP}}$ are the fields due to reflection and diffraction from the half-planes from $x_{1}$ to $-\infty$ and from $x_{2}$ to $\infty$. The field contributions included in the model with LTD half-planes are shown in Figure 3c.

In the code set up to evaluate terrain scattering the currents are computed at discrete, equally spaced points in $x$. If the sample points are $x_{i}$ for $i=1, \ldots, N$, the integral in (11) can be evaluated as

$$
\begin{aligned}
& E_{x}^{s}\left(x_{a}, z_{a}\right)=\sum_{i=1}^{N}\left[g_{x x, i}\left(x_{a}, z_{a}\right) J_{x, i}+g_{x z, i}\left(x_{a}, z_{a}\right) J_{z, i}+f_{x y, i}\left(x_{a}, z_{a}\right) K_{y, i}\right] \\
& E_{y}^{s}\left(x_{a}, z_{a}\right)=\sum_{i=1}^{N}\left[g_{y y, i}\left(x_{a}, z_{a}\right) J_{y, i}+f_{y x, i}\left(x_{a}, z_{a}\right) K_{x, i}+f_{y z, i}\left(x_{a}, z_{a}\right) K_{z, i}\right] \\
& E_{z}^{s}\left(x_{a}, z_{a}\right)=\sum_{i=1}^{N}\left[g_{z x, i}\left(x_{a}, z_{a}\right) J_{x, i}+g_{z z, i}\left(x_{a}, z_{a}\right) J_{z, i}+f_{z y, i}\left(x_{a}, z_{a}\right) K_{y, i}\right] .
\end{aligned}
$$

where $g_{x z, i}\left(x_{a}, z_{a}\right)$ is the component from equation (11) relating the $x$ component of electric field at $\left(x_{a}, z_{a}\right)$ to the $z$ component of electric current over the cell centered at $\left(x_{i}, z_{i}\right)$, and the $f$ factors have the same interpretation for magnetic current. The Green's functions must be integrated over the width of the cell for each $i$, but when the distance from the evaluation 
point $(x, y)$ to the cell at $\left(x_{i}, z_{i}\right)$ is greater than several times the cell width, the value of the Green's function at the center of the cell was simply multiplied by the cell width. For smaller distances an adaptive numerical integration scheme was used.

In the above development a line source at $\left(x_{r}, z_{r}\right)$ has been assumed while the electric field vector was evaluated at the original antenna location $\left(x_{a}, z_{a}\right)$. The solution is equivalent, through reciprocity, with obtaining the components of field $\mathbf{E}^{\mathbf{a}}\left(x_{r}, z_{r}\right) \cdot \mathbf{I}_{r} d \ell$ along the receiving dipole at $\left(x_{r}, z_{r}\right)$ due to three sources at $\left(x_{a}, z_{a}\right)$ with $\mathbf{I}_{a} d \ell$ equal to $\hat{\mathbf{x}}, \hat{\mathbf{y}}$ and $\hat{\mathbf{z}}$. Actual antennas will consist of lengths of wire and possibly conducting surfaces with currents determined by an antenna modeling code such as NEC. If the antenna is described by $M$ wire segments with segment $j$ at $\left(x_{j}, z_{j}\right)$ with length $\Delta_{j}$, direction $\hat{\mathrm{u}}_{j}$ and current $I_{j}$, the total field at the receiver at $\left(x_{r}, z_{r}\right)$ can be obtained by superposing the results of equation (13) for each segment as

$$
E^{r}=\sum_{j=1}^{M} I_{j} \hat{\mathbf{u}}_{j} \cdot \mathbf{E}^{r}\left(x_{j}, z_{j}\right) \Delta_{j}
$$

From (14), (15) and (16), the contribution to $E^{r}$ due to the PO currents can be evaluated as

$$
E^{s}=\sum_{i=1}^{N}\left[G_{x, i} J_{x, i}+G_{y, i} J_{y, i}+G_{z, i} J_{z, i}+F_{x, i} K_{x, i}+F_{y, i} K_{y, i}+F_{z, i} K_{z, i}\right]
$$

where, if the direction of segment $j$ is $\hat{\mathbf{u}}_{j}=u_{x, j} \hat{\mathbf{x}}+u_{y, j} \hat{\mathbf{y}}+u_{z, j} \hat{\mathbf{z}}$ while its image is at $\left(x_{j}^{\prime}, z_{j}^{\prime}\right)$ with direction $\hat{\mathbf{u}}_{j}^{\prime}=u_{x, j}^{\prime} \hat{\mathbf{x}}+u_{y, j}^{\prime} \hat{\mathbf{y}}+u_{z, j}^{\prime} \hat{\mathbf{z}}$, the coefficients are

$$
\begin{aligned}
G_{x, i} & =\sum_{j=1}^{M} I_{j} \Delta_{j}\left\{u_{x, j} g_{x x, i}\left(x_{j}, z_{j}\right)+u_{z, j} g_{z x, i}\left(x_{j}, z_{j}\right)+R_{a}\left[u_{x, j}^{\prime} g_{x x, i}\left(x_{j}^{\prime}, z_{j}^{\prime}\right)+u_{z, j}^{\prime} g_{z x, i}\left(x_{j}^{\prime}, z_{j}^{\prime}\right)\right]\right\} \\
G_{y, i}= & \sum_{j=1}^{M} I_{j} \Delta_{j}\left\{u_{y, j} g_{y y, i}\left(x_{j}, z_{j}\right)+R_{a}\left[u_{y, j}^{\prime} g_{y y, i}\left(x_{j}^{\prime}, z_{j}^{\prime}\right)\right]\right\} \\
G_{z, i}= & \sum_{j=1}^{M} I_{j} \Delta_{j}\left\{u_{x, j} g_{x z, i}\left(x_{j}, z_{j}\right)+u_{z, j} g_{z z, i}\left(x_{j}, z_{j}\right)+R_{a}\left[u_{x, j}^{\prime} g_{x z, i}\left(x_{j}^{\prime}, z_{j}^{\prime}\right)+u_{z, j}^{\prime} g_{z z, i}\left(x_{j}^{\prime}, z_{j}^{\prime}\right)\right]\right\} \\
F_{x, i}= & \sum_{j=1}^{M} I_{j} \Delta_{j}\left\{u_{y, j} f_{y x, i}\left(x_{j}, z_{j}\right)+R_{a}\left[u_{y, j}^{\prime} f_{y x, i}\left(x_{j}^{\prime}, z_{j}^{\prime}\right)\right\}\right. \\
F_{y, i}= & \left.\sum_{j=1}^{M} I_{j} \Delta_{j}\left\{u_{x, j} f_{x y, i}\left(x_{j}, z_{j}\right)+u_{z, j} f_{z y, i}\left(x_{j}, z_{j}\right)+R_{a}\left[u_{x, j}^{\prime} f_{x y, i}\left(x_{j}^{\prime}, z_{j}^{\prime}\right)\right]+u_{z, j}^{\prime} f_{z y, i}\left(x_{j}^{\prime}, z_{j}^{\prime}\right)\right]\right\} \\
F_{z, i}= & \sum_{j=1}^{M} I_{j} \Delta_{j}\left\{u_{y, j} f_{y z, i}\left(x_{j}, z_{j}\right)+R_{a}\left[u_{y, j}^{\prime} f_{y z, i}\left(x_{j}^{\prime}, z_{j}^{\prime}\right)\right]\right\} .
\end{aligned}
$$

Since the $G$ and $F$ factors depend on the antenna, the ground and the index $i$, they can be computed once and saved. Then in computing a radiation pattern, only the far field 
from the source (original receiver) must be computed over the terrain surface to determine the PO currents and the scattered field can be evaluated from (18). The direct and UTD terms in (13) must be evaluated for each antenna segment in summing (17). A disadvantage of interchanging source and receiver is that the blockage of the incident field by the terrain must be determined in evaluating the PO currents for each angle of the pattern, but this can be done quickly by updating a horizon.

\subsection{PO for a 3-Dimensional Surface}

The extension of the 2-D PO model to 3-D is straightforward. The surface will be defined by the function $z=h_{s}(x, y)$ with unit normal vector $\hat{\mathbf{n}}(x, y)$. To determine the reliected fields on the surface it is necessary to resolve the incident field into TE and TM components relative to the plane of incidence on the tangent plane at each point. The vector $\hat{\mathbf{p}}=\left(\hat{\mathbf{r}}_{s} \times \hat{\mathbf{n}}\right) /\left|\hat{\mathbf{r}}_{s} \times \hat{\mathbf{n}}\right|$ will be defined for this purpose, where $\hat{\mathbf{r}}_{s}$ is the unit vector from the source to the point on the surface. If the incident electric and magnetic fields are $\mathbf{E}^{\mathrm{i}}(x, y, z)$ and $\mathbf{H}^{\mathrm{i}}(x, y, z)$, the TE and TM components in the plane of the surface are

$$
\begin{array}{rlrl}
\mathbf{E}_{\mathrm{TE}}^{\mathrm{i}} & =\left(\mathbf{E}^{\mathrm{i}} \cdot \hat{\mathbf{p}}\right) \hat{\mathbf{p}}, \quad \text { and } & \mathbf{E}_{\mathrm{TM}}^{\mathrm{i}}=\mathbf{E}^{\mathrm{i}}-\left(\mathbf{E}^{\mathrm{i}} \cdot \hat{\mathbf{p}}\right) \hat{\mathbf{p}}-\left(\mathbf{E}^{\mathrm{i}} \cdot \hat{\mathbf{n}}\right) \hat{\mathbf{n}} \\
\mathbf{H}_{\mathrm{TM}}^{\mathrm{i}}=\left(\mathbf{H}^{\mathrm{i}} \cdot \hat{\mathbf{p}}\right) \hat{\mathbf{p}}, \quad \text { and } & \mathbf{H}_{\mathrm{TE}}^{\mathrm{i}}=\mathbf{H}^{\mathbf{i}}-\left(\mathbf{H}^{\mathbf{i}} \cdot \hat{\mathbf{p}}\right) \hat{\mathbf{p}}-\left(\mathbf{H}^{\mathrm{i}} \cdot \hat{\mathbf{n}}\right) \hat{\mathbf{n}}
\end{array}
$$

The sums of incident and reflected fields on the surface are then

$$
\begin{aligned}
\mathbf{E}_{\mathrm{TE}}^{t} & =\left(1+R_{\mathrm{TE}}\right)\left(\mathbf{E}^{\mathrm{i}} \cdot \hat{\mathbf{p}}\right) \hat{\mathbf{p}} \\
\mathbf{E}_{\mathrm{TM}}^{t} & =\left(1-R_{\mathrm{TM}}\right)\left[\mathbf{E}^{\mathrm{i}}-\left(\mathbf{E}^{\mathrm{i}} \cdot \hat{\mathbf{p}}\right) \hat{\mathbf{p}}-\left(\mathbf{E}^{\mathrm{i}} \cdot \hat{\mathbf{n}}\right) \hat{\mathbf{n}}\right] \\
\mathbf{H}_{\mathrm{TM}}^{t} & =\left(1+R_{\mathrm{TM}}\right)\left(\mathbf{H}^{\mathrm{i}} \cdot \hat{\mathbf{p}}\right) \hat{\mathbf{p}} \\
\mathbf{H}_{\mathrm{TE}}^{t} & =\left(1-R_{\mathrm{TE}}\right)\left[\mathbf{H}^{\mathrm{i}}-\left(\mathbf{H}^{\mathrm{i}} \cdot \hat{\mathbf{p}}\right) \hat{\mathbf{p}}-\left(\mathbf{H}^{\mathrm{i}} \cdot \hat{\mathbf{n}}\right) \hat{\mathbf{n}}\right]
\end{aligned}
$$

and the total surface fields are

$$
\begin{aligned}
& \mathbf{E}^{t}=\mathbf{E}_{\mathrm{TE}}^{t}+\mathbf{E}_{\mathrm{TM}}^{t}=\left(1-R_{\mathrm{TM}}\right) \mathbf{E}^{\mathrm{i}}+\left(R_{\mathrm{TE}}+R_{\mathrm{TM}}\right)\left(\mathbf{E}^{\mathrm{i}} \cdot \hat{\mathbf{p}}\right) \hat{\mathbf{p}}-\left(1-R_{\mathrm{TM}}\right)\left(\mathbf{E}^{\mathrm{i}} \cdot \hat{\mathbf{n}}\right) \hat{\mathbf{n}} \\
& \mathbf{H}^{t}=\mathbf{H}_{\mathrm{TE}}^{t}+\mathbf{H}_{\mathrm{TM}}^{t}=\left(1-R_{\mathrm{TE}}\right) \mathbf{H}^{\mathrm{i}}+\left(R_{\mathrm{TE}}+R_{\mathrm{TM}}\right)\left(\mathbf{H}^{\mathrm{i}} \cdot \hat{\mathbf{p}}\right) \hat{\mathbf{p}}-\left(1-R_{\mathrm{TE}}\right)\left(\mathbf{H}^{\mathrm{i}} \cdot \hat{\mathbf{n}}\right) \hat{\mathbf{n}} .
\end{aligned}
$$

With the condition that the fields are zero behind the surface, the electric and magnetic surface currents are

$$
\begin{aligned}
\mathbf{K}(x, y) & =-\hat{\mathbf{n}} \times \mathbf{E}^{t}=-\left(1-R_{\mathrm{TM}}\right)\left(\hat{\mathbf{n}} \times \mathbf{E}^{\mathrm{i}}\right)-\left(R_{\mathrm{TE}}+R_{\mathrm{TM}}\right)\left(\mathbf{E}^{\mathrm{i}} \cdot \hat{\mathbf{p}}\right)(\hat{\mathbf{n}} \times \hat{\mathbf{p}}) \\
\mathbf{J}(x, y) & =\hat{\mathbf{n}} \times \mathbf{H}^{t}=\left(1-\Gamma_{\mathrm{TE}}\right)\left(\hat{\mathbf{n}} \times \mathbf{H}^{\mathrm{i}}\right)+\left(R_{\mathrm{TE}}+R_{\mathrm{TM}}\right)\left(\mathbf{H}^{\mathrm{i}} \cdot \hat{\mathbf{p}}\right)(\hat{\mathbf{n}} \times \hat{\mathbf{p}})
\end{aligned}
$$

If the PO currents are computed over a rectangular region with $x_{1} \leq x \leq x_{2}$ and $y_{1} \leq y \leq y_{2}$ the scattered field is

$$
\mathbf{E}^{s}(x, y, z)=-\int_{x_{1}}^{x_{2}} \int_{y_{1}}^{y_{2}}\left[j \omega \mu \overline{\bar{\Gamma}}\left(\mathbf{r}, \mathbf{r}^{\prime}\right) \cdot \mathbf{J}\left(x^{\prime}\right)+\mathbf{K}\left(x^{\prime}\right) \times \nabla G\left(\mathbf{r}, \mathbf{r}^{\prime}\right)\right]\left(\hat{\mathbf{z}} \cdot \hat{\mathbf{n}}^{\prime}\right)^{-1} d x^{\prime} d y^{\prime}
$$


where $(\hat{\mathbf{z}} \cdot \hat{\mathbf{n}})^{-1} d x^{\prime} d y^{\prime}$ is the element of area on the surface. $\overline{\bar{\Gamma}}\left(\mathbf{r}, \mathbf{r}^{\prime}\right)$ is the dyadic Green's function

$$
\begin{aligned}
& \overline{\bar{\Gamma}}\left(\mathbf{r}, \mathbf{r}^{\prime}\right)=\left(\overline{\overline{\mathbf{I}}}+\frac{1}{k^{2}} \nabla \nabla\right) G\left(\mathbf{r}, \mathbf{r}^{\prime}\right) \\
& G\left(\mathbf{r}, \mathbf{r}^{\prime}\right)=\frac{e^{-j k\left|\mathbf{r}-\mathbf{r}^{\prime}\right|}}{4 \pi\left|\mathbf{r}-\mathbf{r}^{\prime}\right|}
\end{aligned}
$$

with $\mathbf{r}=x \hat{\mathbf{x}}+y \hat{\mathbf{y}}+z \hat{\mathbf{z}}$ the vector to the field evaluation point and $\mathbf{r}^{\prime}=x^{\prime} \hat{\mathbf{x}}+y^{\prime} \hat{\mathbf{y}}+h_{s}\left(x^{\prime}, y^{\prime}\right) \hat{\mathbf{z}}$ the vector to the source point on the surface. The limits in $x$ and $y$ should be positive and negative infinity, but in practice the integral is truncated at points beyond which the contributions should be negligible.

The second-order corrections for source and receiver, as illustrated in Figure $3 c$, were also included in the 3-D model. The UTD half-planes to infinity in the 2-D model become a rectangular frame around the PO region in 3-D. It was assumed that the points on the boundary of the PO region are at a constant height of $z_{g}$. Then the reflected and diffracted fields from the frame are obtained from four horizontal half-planes, each of which may contribute a diffracted field if the diffraction point falls within the limits of the aperture. The more complicated results from [13] for diffraction at oblique incidence to an edge must be used. The UTD extension of the plane to infinity significantly reduces the ripple produced in radiation patterns due to truncating the PO integral. Although the UTD for oblique incidence is more complicated than in $2-\mathrm{D}$, the time saved in computing the surface integral makes its use worthwhile.

\section{THE 2-D INTEGRAL EQUATION MODEL}

Integral equation solutions for the current on the terrain surface were developed as a means to check the validity of the PO results. From Poggio and Miller [14], the integral equations for electric and magnetic fields with $\mathbf{r}$ ranging over a surface $S$ are

$$
\mathbf{E}(\mathbf{r})=T \mathbf{E}^{i}(\mathbf{r})-T f_{S}\left[j \omega \mu\left(\hat{\mathbf{n}}^{\prime} \times \mathbf{H}\right) G-\left(\hat{\mathbf{n}}^{\prime} \times \mathbf{E}\right) \times \nabla^{\prime} G-\left(\hat{\mathbf{n}}^{\prime} \cdot \mathbf{E}\right) \nabla^{\prime} G\right] d s^{\prime}
$$

and

$$
\mathbf{H}(\mathbf{r})=T \mathbf{H}^{i}(\mathbf{r})+T f_{S}\left[j \omega \epsilon\left(\hat{\mathbf{n}}^{\prime} \times \mathbf{E}\right) G+\left(\hat{\mathbf{n}}^{\prime} \times \mathbf{H}\right) \times \nabla^{\prime} G+\left(\hat{\mathbf{n}}^{\prime} \cdot \mathbf{H}\right) \nabla^{\prime} G\right] d s^{\prime}
$$

where $T=(1-\Omega / 4 \pi)^{-1}$ and $\Omega$ is the solid angle subtended by the surface at $\mathbf{r}$ in the limit as $\mathbf{r}$ approaches the surface from the outside. A smooth surface will be assumed from here on, so $\Omega=2 \pi$ and $T=2$.

The third term in each integral, which may be written in terms of an equivalent current with second derivatives on the Green's function, can cause difficulties in the numerical solution due to the order of the singularity. However, in a 2-D problem the last term in (22) vanishes for TE polarization, and the last term in (23) vanishes for TM polarization. Then 
using the surface currents $K_{s}=E_{y}, K_{y}=-E_{s}, J_{s}=-H_{y}$ and $J_{y}=H_{s}$ with the coordinates shown in Figure 1 and $\mathbf{r}=x \hat{\mathbf{x}}+h_{s}(x) \hat{\mathbf{z}}$, the equations become for TE polarization

$$
E_{y}^{i}(\mathbf{r})=\frac{1}{2} K_{s}(x)+\int_{x_{m}}^{x_{p}}\left[j \omega \mu J_{y}\left(x^{\prime}\right) G_{c}-K_{s}\left(x^{\prime}\right) \frac{\partial}{\partial n^{\prime}} G_{c}\right]\left(\hat{\mathbf{z}} \cdot \hat{\mathbf{n}}^{\prime}\right)^{-1} d x^{\prime}
$$

and for TM polarization

$$
H_{y}^{i}(\mathbf{r})=-\frac{1}{2} J_{s}(x)+\int_{x_{m}}^{x_{p}}\left[j \omega \epsilon K_{y}\left(x^{\prime}\right) G_{c}+J_{s}\left(x^{\prime}\right) \frac{\partial}{\partial n^{\prime}} G_{c}\right]\left(\hat{\mathbf{z}} \cdot \hat{\mathbf{n}}^{\prime}\right)^{-1} d x^{\prime}
$$

where $J_{y}\left(x^{\prime}\right)$ represents the $y$ component of electric current at $\mathbf{r}^{\prime}=x^{\prime} \hat{\mathbf{x}}+h_{s}\left(x^{\prime}\right) \hat{\mathbf{z}}$ on the surface and $\partial G_{c} / \partial n^{\prime}=\hat{\mathbf{n}}^{\prime} \cdot \nabla^{\prime} G_{c}$. The 2-D Green's function is

$$
G_{c}\left(\mathbf{r}, \mathbf{r}^{\prime}\right)=\frac{-j}{4} H_{0}^{(2)}\left(k\left|\mathbf{r}-\mathbf{r}^{\prime}\right|\right)
$$

Although equations (24) and (25) each involve two unknown currents, the Leontovich impedance boundary condition can be applied to eliminate one unknown in each case. With $K_{s} / J_{y}=-K_{y} / J_{s}=\eta_{g}$, the equation for TE polarization becomes

$$
E_{y}^{i}(\mathbf{r})=\frac{1}{2} K_{s}(x)-\int_{x_{m}}^{x_{p}} K_{s}\left(x^{\prime}\right)\left(-j \omega \mu / \eta_{g}+\frac{\partial}{\partial n^{\prime}}\right) G_{c}\left(\mathbf{r}, \mathbf{r}^{\prime}\right)\left(\hat{\mathbf{z}} \cdot \hat{\mathbf{n}}^{\prime}\right)^{-1} d x^{\prime}
$$

and for TM polarization

$$
H_{y}^{i}(\mathbf{r})=-\frac{1}{2} J_{s}(x)+\int_{x_{m}}^{x_{p}} J_{s}\left(x^{\prime}\right)\left(-j \omega \epsilon \eta_{g}+\frac{\partial}{\partial n^{\prime}}\right) G_{c}\left(\mathbf{r}, \mathbf{r}^{\prime}\right)\left(\hat{\mathbf{z}} \cdot \hat{\mathbf{n}}^{\prime}\right)^{-1} d x^{\prime}
$$

where $x_{m} \leq x \leq x_{p}$. The impedance boundary condition is generally accurate as long as the radius of curvature of the surface is much greater than the skin depth in the medium.

Equations (26) and (27) are related through duality, so only one code is needed to solve them. The only difference in the solutions is that the source of $E_{y}^{i}$ in (26) will be an electric line current in the $y$ direction, while the source of $H_{y}^{i}$ in (27) will be a strip of vertical electric current $J_{z}$ extending over $-\infty<y<\infty$.

The numerical solution will be considered for equation (27). The equation was solved by means of the method of moments with pulse basis functions and point matching. The current is expanded in a set of $N$ basis functions $f_{j}(x)$ as

$$
J_{s}(x)=\sum_{j=1}^{N} J_{j} f_{j}(x)
$$

Substituting (28) into (27) and enforcing the equality at match points $x_{i}=x_{m}+(i-1) \Delta x$ for $i=1, \ldots, N$ and $\Delta x=\left(x_{p}-x_{m}\right) /(N-1)$ yields the set of linear equations

$$
\sum_{j=1}^{N} G_{i j} J_{j}=E_{i}^{i}, \quad i=1, \ldots, N
$$


where

$$
G_{i j}=-\frac{1}{2} \delta_{i j}+\int_{x_{m}}^{x_{p}} f_{j}\left(x^{\prime}\right)\left(-j \omega \epsilon \eta_{g}+\frac{\partial}{\partial n^{\prime}}\right) G_{c}\left(\mathbf{r}_{\mathbf{i}}, \mathbf{r}^{\prime}\right)\left(\hat{\mathbf{z}} \cdot \hat{\mathbf{n}}^{\prime}\right)^{-1} d x^{\prime}
$$

The excitation vector is $E_{i}^{i}=E_{y}^{i}\left(\mathbf{r}_{i}\right)$ and $\delta_{i j}=1$ if $i=j$ or 0 otherwise. The current can be obtained by solving the matrix equation or inverting the matrix $\left[G_{i j}\right]$ as

$$
\left[J_{i}\right]=\left[G_{i j}\right]^{-1}\left[E_{j}^{i}\right]
$$

Simple pulse basis functions can be used for the current expansion, since the kernel of the integral equation is relatively well behaved. However, if the range of the integral equation is truncated at finite limits of $x_{m}$ and $x_{p}$, reflections will be introduced into the solution for current. To reduce these end reflections the first and last basis functions were extended to infinity with the form of a decaying exponential function $\exp \left[-j k R_{s}(x)\right] / R_{s}^{n}(x)$, where $R_{s}(x)=\left[\left(x-x_{a}\right)^{2}+\left(z_{s}-z_{a}\right)^{2}\right]^{1 / 2}$ is the distance from the point $\left(x, z_{s}\right)$ on the surface to the source of the exciting fields $E^{i}$ and $H^{i}$ at $\left(x_{a}, z_{a}\right)$. This form can be used even over parts of the surface not directly illuminated by the source. The exponent $n$ was chosen as $1 / 2$ for vertical polarization in equation (27) and $3 / 2$ for horizontal polarization in (26). These choices do not seem to be critical as long as continuity of current is maintained. The pulse basis functions for $j=2, \ldots, N-1$ are defined as

$$
f_{j}(x)= \begin{cases}1, & \text { if } x_{j}-\Delta x / 2<x \leq x_{j}+\Delta x / 2 \\ 0, & \text { otherwise }\end{cases}
$$

For the first and last basis functions,

$$
f_{1}(x)= \begin{cases}\frac{e^{-j k\left[R_{s}(x)-R_{s}\left(x_{1}\right)\right]}}{\left[R_{s}(x) / R_{s}\left(x_{1}\right)\right]^{n}}, & \text { if } x \leq x_{1}-\Delta x / 2 \\ 1, & \text { if } x_{1}-\Delta x / 2<x \leq x_{1}+\Delta x / 2 \\ 0, & \text { if } x>x_{1}+\Delta x / 2\end{cases}
$$

and

$$
f_{N}(x)= \begin{cases}0, & \text { if } x<x_{N}-\Delta x / 2 \\ 1, & \text { if } x_{N}-\Delta x / 2<x \leq x_{N}+\Delta x / 2 \\ \frac{e^{-j k\left[R_{s}(x)-R_{s}\left(x_{N}\right)\right]}}{\left[R_{s}(x) / R_{s}\left(x_{N}\right)\right]^{n}}, & \text { if } x>x_{N}+\Delta x / 2 .\end{cases}
$$

The exponential functions start at the outer edges of the first and last pulse functions, but are normalized to unity at the centers of the pulses so that they represent smooth extensions of the stair-stepped approximation formed by the pulses.

For the pulse basis function, the integral in (29) was approximated by evaluating the integrand at $x_{j}$ and multiplying by $\Delta x$ as long as $\left|\mathbf{r}_{i}-\mathbf{r}_{j}\right|>6 \Delta x$. For smaller separations, the singular and discontinuous terms were subtracted from the integrand and integrated analytically, and three-point Gaussian quadrature was used on the remainder.

When $f_{1}(x)$ or $f_{N}(x)$ is substituted into (29) the lower or upper limit of the integral must be extended to $-\infty$ or $+\infty$, respectively. The integrals of the exponential functions oscillate 
and converge relatively slowly along the real $x$ axis. However, the integration contour can be deformed into the complex $x$ plane to obtain a rapid exponential convergence. $R_{s}(x)$ has branch points at $x=x_{a} \pm j\left(z-z_{a}\right)$, but with the restriction that the source is located between the limits of the integral equation range, $x_{m}<x_{a}<x_{p}$, the branch cuts are not intercepted in deforming the contour. The integrals of the first and last basis functions are then evaluated as

$$
\begin{aligned}
G_{i 1}= & -\frac{1}{2} \delta_{i 1}+\int_{x_{1}-\Delta x / 2}^{x_{1}+\Delta x / 2}\left(-j \omega \epsilon \eta_{g}+\frac{\partial}{\partial n^{\prime}}\right) G_{c}\left(\mathbf{r}_{\mathbf{i}}, \mathbf{r}^{\prime}\right)\left(\hat{\mathbf{z}} \cdot \hat{\mathbf{n}}^{\prime}\right)^{-1} d x^{\prime} \\
& +\int_{x_{1}-\Delta x / 2+j \infty}^{x_{1}-\Delta x / 2} \frac{e^{-j k\left[R_{s}(x)-R_{a}\left(x_{1}\right)\right]}}{\left[R_{s}(x) / R_{s}\left(x_{1}\right)\right]^{n}}\left(-j \omega \epsilon \eta_{g}+\frac{\partial}{\partial z}\right) G_{c}\left(\mathbf{r}_{\mathbf{i}}, \mathbf{r}^{\prime}\right) d x^{\prime}
\end{aligned}
$$

and

$$
\begin{aligned}
G_{i N}= & -\frac{1}{2} \delta_{i N}+\int_{x_{N}-\Delta x / 2}^{x_{N}+\Delta x / 2}\left(-j \omega \epsilon \eta_{g}+\frac{\partial}{\partial n^{\prime}}\right) G_{c}\left(\mathbf{r}_{\mathbf{i}}, \mathbf{r}^{\prime}\right)\left(\hat{\mathbf{z}} \cdot \hat{\mathbf{n}}^{\prime}\right)^{-1} d x^{\prime} \\
& +\int_{x_{N}+\Delta x / 2}^{x_{N}+\Delta x / 2-j \infty} \frac{e^{-j k\left[R_{s}(x)-R_{s}\left(x_{N}\right)\right]}}{\left[R_{s}(x) / R_{s}\left(x_{N}\right)\right]^{n}}\left(-j \omega \epsilon \eta_{g}+\frac{\partial}{\partial z}\right) G_{c}\left(\mathbf{r}_{\mathbf{i}}, \mathbf{r}^{\prime}\right) d x^{\prime} .
\end{aligned}
$$

The integrals to infinity were evaluated numerically with an adaptive Romberg quadrature routine [15]. Typically fewer than 20 integrand evaluations are needed since both the exponential function and the Hankel function in $G_{c}$ decay exponentially on the deformed contours.

When the source point $x_{a}$ falls outside the range of the integral equation, the evaluation of the integrals to infinity becomes more difficult since a branch cut may be encountered in deforming the contour. Also, a saddle point may lie near the integration limit, in which case the initial convergence of the integrand will be slow. The same problems occur in evaluating the integral for the radiated field due to the current, since the evaluation points for radiated field will usually lie outside of the range $x_{m}<x<x_{p}$. These problems could be handled by developing asymptotic approximations or simply by working harder at the numerical integration. However, there was not time to pursue these problems in the present effort. In evaluating the radiated field, the contributions from the exponential extensions of $f_{1}$ and $f_{N}$ were simply ignored. Hence the limits $x_{m}$ and $x_{p}$ were located as far as possible from the scattering region to minimize the ripples in the radiation pattern.

In the special case of scattering by raised regions on a perfectly conducting surface the flat surface extending to infinity can be eliminated by including the image of the scattering region. The integrals in (26) and (27) then include the currents on the raised surface and their images, and the exciting fields $E^{i}$ and $H^{i}$ include the fields of the source and its image. The image currents and source also contribute to the radiated field. The pulse functions of (30) can be used for all basis functions in this case. Codes were also developed to solve (26) and (27) for perfectly conducting ground since the solution range can be truncated at the points where the scattering surface meets the flat ground $(z=0)$ without concern for perturbations of the current or radiation patterns. 


\section{NUMERICAL RESULTS AND VALIDATION}

Initial validation of the PO codes was based on comparisons with results from the integral equation codes. Hence, the validation of the integral equation solutions will be considered first. A similar integral equation code was developed by Janaswamy [16], and some of the results from that report were useful for validating our codes. In addition, the program WAGNER, developed by Ott [9], was used for validation. WAGNER solves a Volterra integral equation derived from the parabolic approximation of the wave equation. It is a 2-D model, and neglects backscattering, but is reasonably well validated and has been widely used for a number of years. The surface field from Wagner is probably of sufficient accuracy to be used directly to obtain radiation patterns for validating PO. However, the field would need to be extended into the near-field region of sources near ground. Also, Ott has recently developed a new code RING [17] which gives different results in some cases than WAGNER, so there were questions about which code was correct.

The first test of the integral equation code was for a vertical strip of electric current over a flat ground plane. An exact solution for the field over ground is available from Wait $[18]$ as

$$
H_{y}(x, z)=2 H_{y}^{i}(x, z)-\frac{j k_{0} \Delta_{s}}{\pi} \int_{0}^{\infty} \frac{\xi \sin \left[\xi\left(x-x_{a}\right)\right] e^{-\tau\left(z+z_{a}\right)}}{\tau\left(\tau+j k_{0} \Delta_{s}\right)} d \xi
$$

where $\tau^{2}=\xi^{2}-k_{0}^{2}$, and $\Delta_{g}=\eta_{g} / \eta_{0}$ is the normalized surface impedance. $H_{y}^{i}(x, z)$ is the incident field from the source at $\left(x_{a}, z_{a}\right)$, which for a vertical strip of electric current is

$$
H_{y}^{i}(x, z)=-\frac{j k_{0}}{4} \frac{\left(x-x_{a}\right)}{R_{a}} H_{1}^{(2)}\left(k_{0} R_{a}\right)
$$

where $R_{a}=\left[\left(x-x_{a}\right)^{2}+\left(z-z_{a}\right)^{2}\right]^{1 / 2}$ and $H_{1}^{(2)}$ is the Hankel function.

The problem modeled was a source at $x_{a}=0$ and $z_{a}=0.03 \lambda_{0}$ over a ground with $\tilde{\epsilon}_{g}=15-j 6$. The normalized attenuation function along the ground, $\left|H_{y}(x) / 2 H_{y}^{i}(x)\right|$, is plotted in Figure 4, where the solid curve is the result of solving integral equation (27) with 200 unknowns over the range $-\lambda_{0} \leq x \leq 2 \lambda_{0}$. The result from evaluating (31) is plotted as a line with long dashes, but is mostly not visible since it lies on top of the solid line. When the number of unknowns was reduced to 60 , errors of about one percent were seen in the integral equation solution. The visible line with short dashes in Figure 4 is the result of solving the integral equation without the extensions of the first and last basis functions to infinity, so reflections are seen at the ends. Much largor reflections occur for horizontal polarization, but in either case the reflections are essen : „ly eliminated with the extended basis functions.

The next test of the integral equation code was for a $1 \mathrm{~km}$ high Gaussian hill with a source at a distance of $5 \mathrm{~km}$. The 2-D line source is a vertical strip of electric current at $x_{a}=0$ and $z_{a}=1 \mathrm{~m}$. This is somewhat of a classic problem, having been solved by Berry $[19]$, Ott $[9,10,20]$ and Janaswamy [16] among others. The Gaussian hill is defined by

$$
h_{s}(x)=H e^{-9(x-c)^{2} / w^{2}}
$$




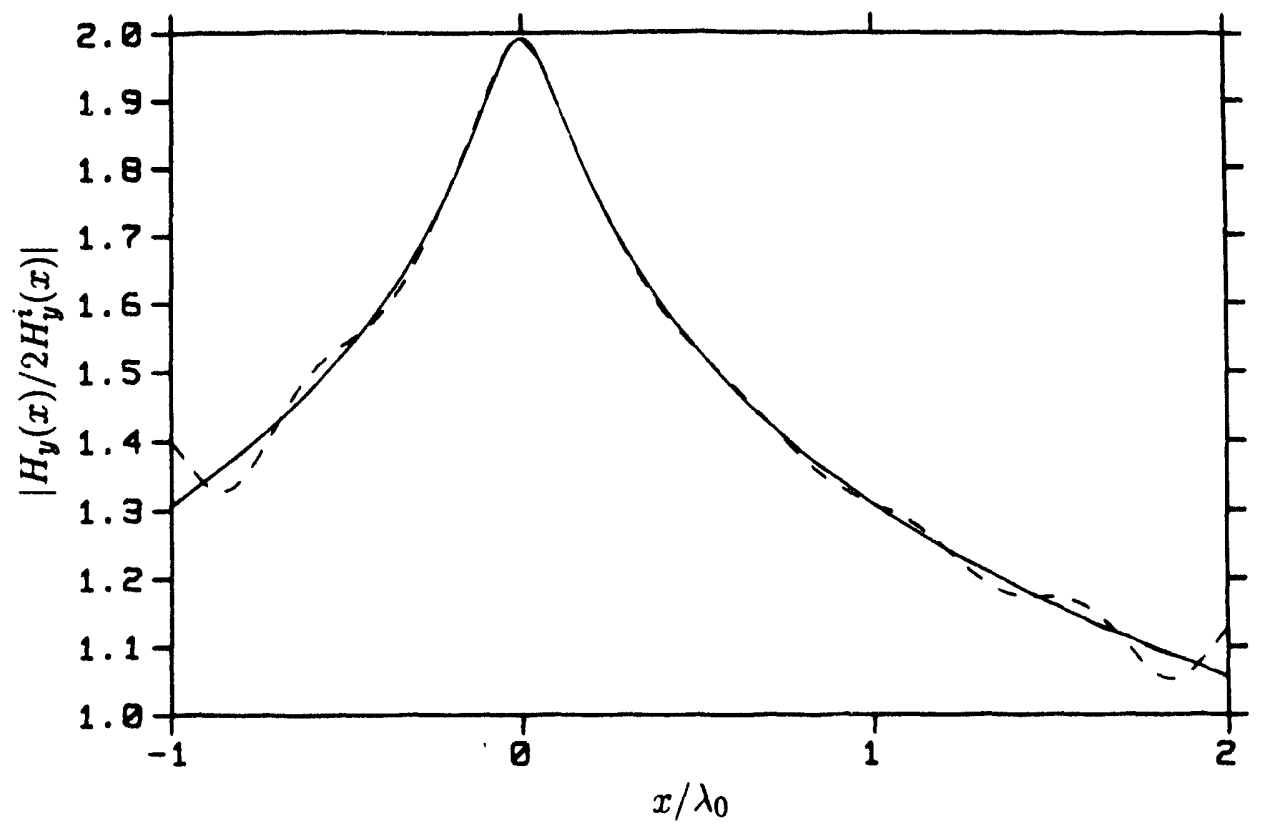

Fig. 4. Solution for the normalized field $H_{y}$ for a source at height $z=0.03 \lambda_{0}$ over a flat ground with $\tilde{\epsilon}_{g}=15-j 6$. The solution of the integral equation with basis functions extended to $\pm \infty(-)$ and the result of equation (31) (- $-\longrightarrow$ coincide. The solution without extended basis functions $\left(_{-}--_{-}\right)$ shows reflections at the ends of the range.

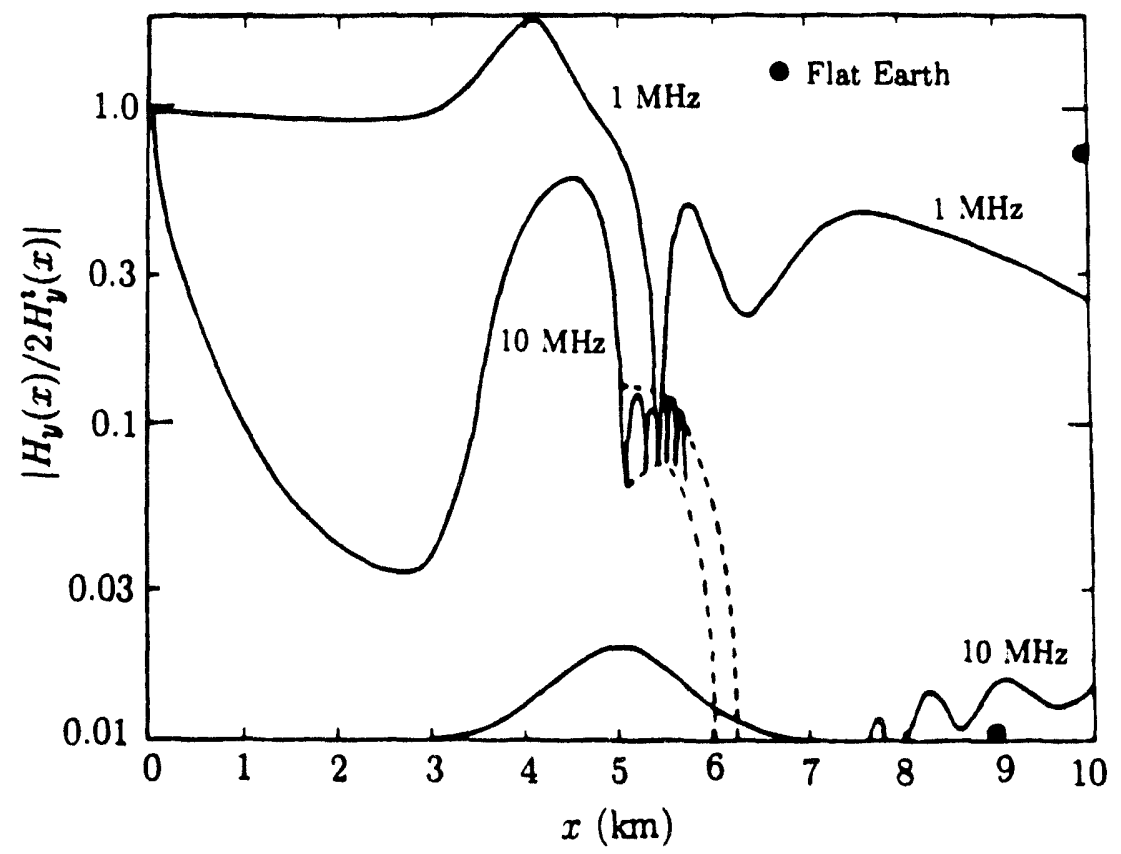

Fig. 5. Normalized field $H_{y}$ over the surface of a Gaussian hill from Berry's solution of Hufford's integral equation. (Source: L. A. Berry, [19], IEEE (c) 1967. Reproduced with permission of IEEE.)

with $H=1 \mathrm{~km}, c=5 \mathrm{~km}$ and $w=3 \mathrm{~km}$, and the ground parameters are $\epsilon_{r}=10$ and $\sigma=0.01$ $\mathrm{S} / \mathrm{m}$. Berry [19] computed the field over the hill by solving Hufford's integral equation [8] at 1 and $10 \mathrm{MHz}$, with the results reproduced as Figure 5 in this report. Hufford's equation is a Volterra integral equation derived from the free space Green's function. Ott and Berry 


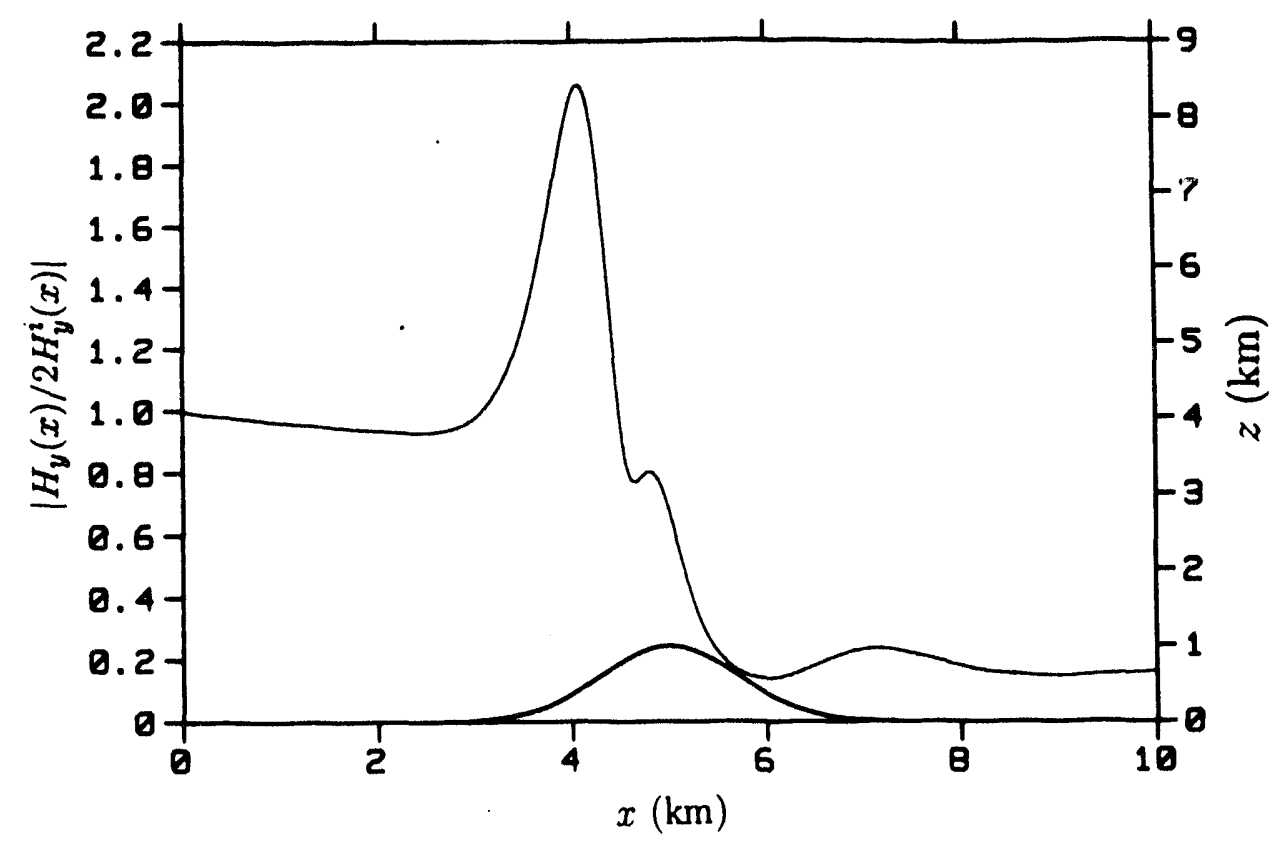

Fig. 6. Result from program WAGNER for the normalized $H_{y}$ over the surface $o^{\prime}$ the Gaussian hill at 1 $\mathrm{MHz}$.

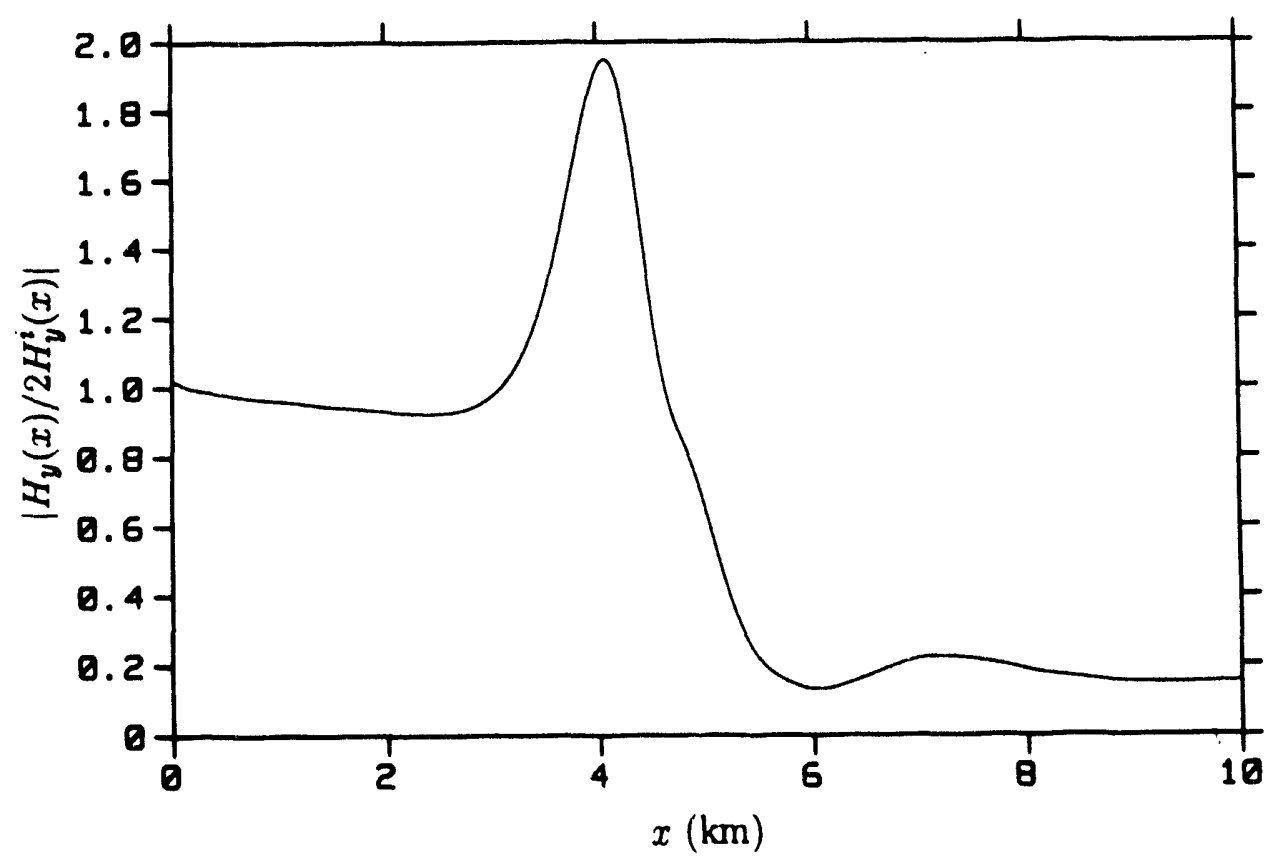

Fig. 7. Integral equation solution for the normalized $H_{y}$ over the surface of the Gaussian hill at $1 \mathrm{MHz}$.

[9] developed a new integral equation using a modified Sommerfeld attenuation function as an elementary function. This alternate equation, which Ott used in the code WAGNER, is easier to solve than Hufford's but apparently becomes unstable at high frequencies [21]. The result of running WAGNER for the Gaussian hill at $1 \mathrm{MHz}$ is shown in Figure 6.

The solution of the integral equation (27) for the Gaussian hill at $1 \mathrm{MHz}$ is shown in Figure 7. This solution used 1000 unknowns over the range $-0.5 \mathrm{~km} \leq x \leq 10 \mathrm{~km}$, although 
the result with 500 unknowns over this range was essentially the same. The integral equation solution is in reasonable agreement with the solutions of Hufford's equation and WAGNER. Each shows the characteristic peak on the near side of the hill due to height gain and focusing. The field decreases toward the top of the hill, and is small behind the hill, with some recovery at larger distances. Program WAGNFR predicts a more pronounced secondary peak near the top of the hill, while the integral equation solution is closer to Berry's solution of Hufford's equation in this region, showing only a slight shoulder in the curve. Janaswamy's solution of the integral equation [16] also shows a clear second peak, but it occurs at a higher level, around $x=4.5 \mathrm{~km}$, while that in the WAGNER solution is at $x=4.78 \mathrm{~km}$. This second peak may be a developing feature in the current at $1 \mathrm{MHz}$, and very sensitive to the solution method. Results from the integral equation code and WAGNER for the Gaussian hill at 3 $\mathrm{MHz}$ are shown in Figure 8, and are in good agreement.

The integral equation solution for the Gaussian hill at $10 \mathrm{MHz}$ is shown in Figure 9. The equation was solved with 1400 and 3000 unknowns over the range $-0.5 \mathrm{~km} \leq x \leq 10 \mathrm{~km}$. With 1400 unknowns $\left(\Delta x=0.25 \lambda_{0}\right)$ the low-level field behind the hill is inaccurate, but the field on the front of the hill is close to that obtained with 3000 unknowns. The main features of the integral equation solution are in agreement with Berry's solution of Hufford's equation in Figure 5. The nulls in the region of $x=6 \mathrm{~km}$ are the result of interference between a creeping wave and field diffracted over the hill. In this range Berry's result is larger than the apparently accurate result with 3000 unknowns in Figure 9. It seems likely that Berry also had problems with sampling accuracy, since his solution was severely straining the computing power available at the time. At greater distances behind the hill the normalized field recovers as the diffracted field becomes stronger. From 8 to $10 \mathrm{~km}$ Berry's result and the integral equation solution with 3000 unknowns are again in good agreement.

The integral equation result for the Gaussian hill at $30 \mathrm{MHz}$ is shown in Figure 10 . The equation was solved over the range $-0.5 \mathrm{~km} \leq x \leq 7 \mathrm{~km}$ with 3000 unknowns. With $\Delta x=0.25 \lambda_{0}$, the low-level fields behind the hill are not expected to be accurate. However, the main features of the solution are similar to those seen at lower frequencies and probably are accurate. Some low-level numerical noise can be seen in the solution since the 3000-order matrix was solved in single precision (32 bits).

Radiation patterns obtained from the integral equation solution and 2-D PO are compared in Figure 11a for the vertical source and Gaussian hill at $1 \mathrm{MHz}$. The integral equation was solved over the range $-15 \mathrm{~km} \leq x \leq 10 \mathrm{~km}$ with 1000 unknowns so that the current is truncated at reasonably distant points. The PO solution included the terms depicted in Figure $3 \mathrm{c}$ and equation (13), with PO currents computed over the range $1.5 \mathrm{~km} \leq x \leq 8.5$ $\mathrm{km}$ with 300 integration points. The integral equation result shows some ripple due to trincating the current. The small peak near $\theta=80^{\circ}$ in the PO result is probably an artifact, as the shadow boundary is passing under the evaluation point at $x=0$. The geometrical optics (GO) solution using Ray Launching [2] for this case is shown in Figure 11b. A similar interference pattern is seen in the GO result, but it is limited between a caustic at $19^{\circ}$ and the shadow boundary. The relative amplitude of the interference pattern is somewhat less in the $\mathrm{GO}$ result, probably because the $\mathrm{GO}$ model is a Gaussian ridge with a point source, while the integral equation and 2-D PO use line sources. This difference should be less noticeable 
a)

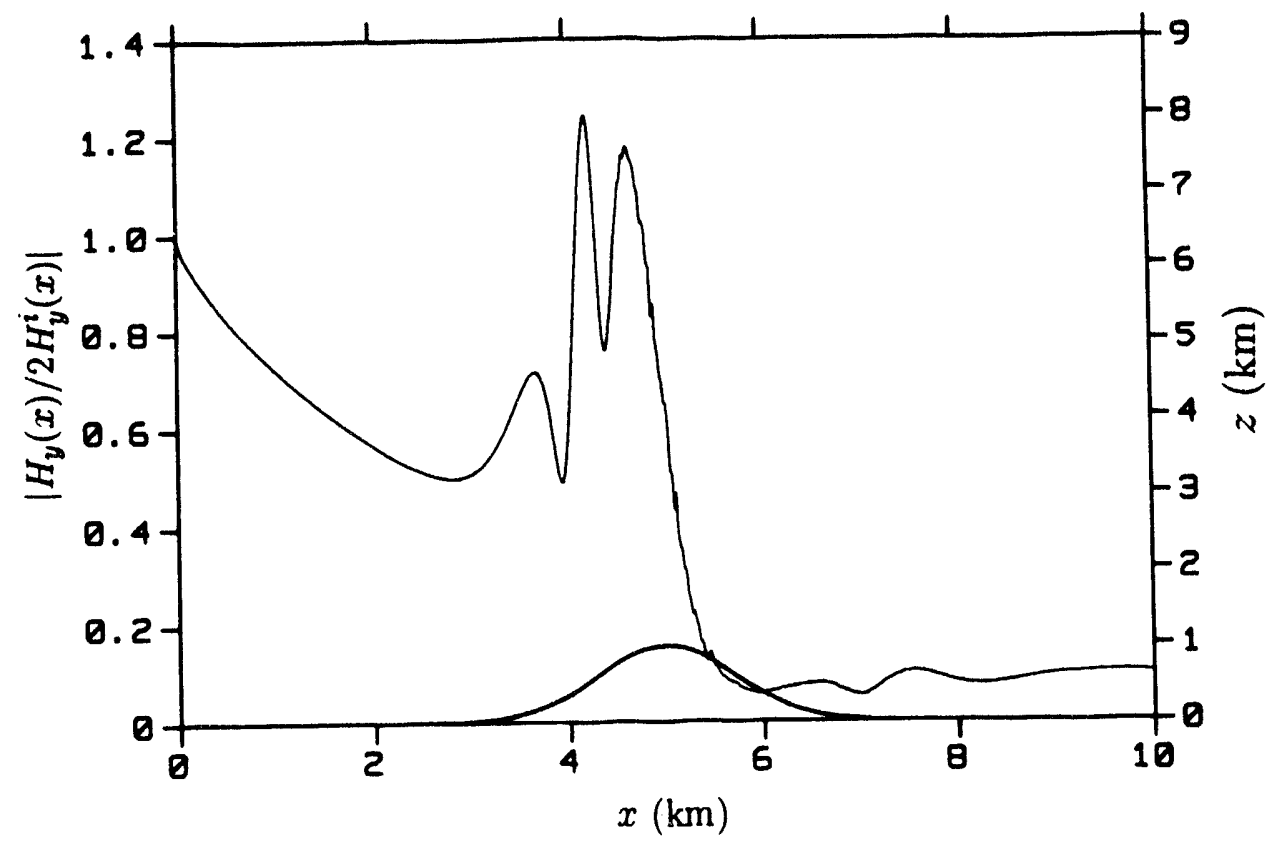

b)

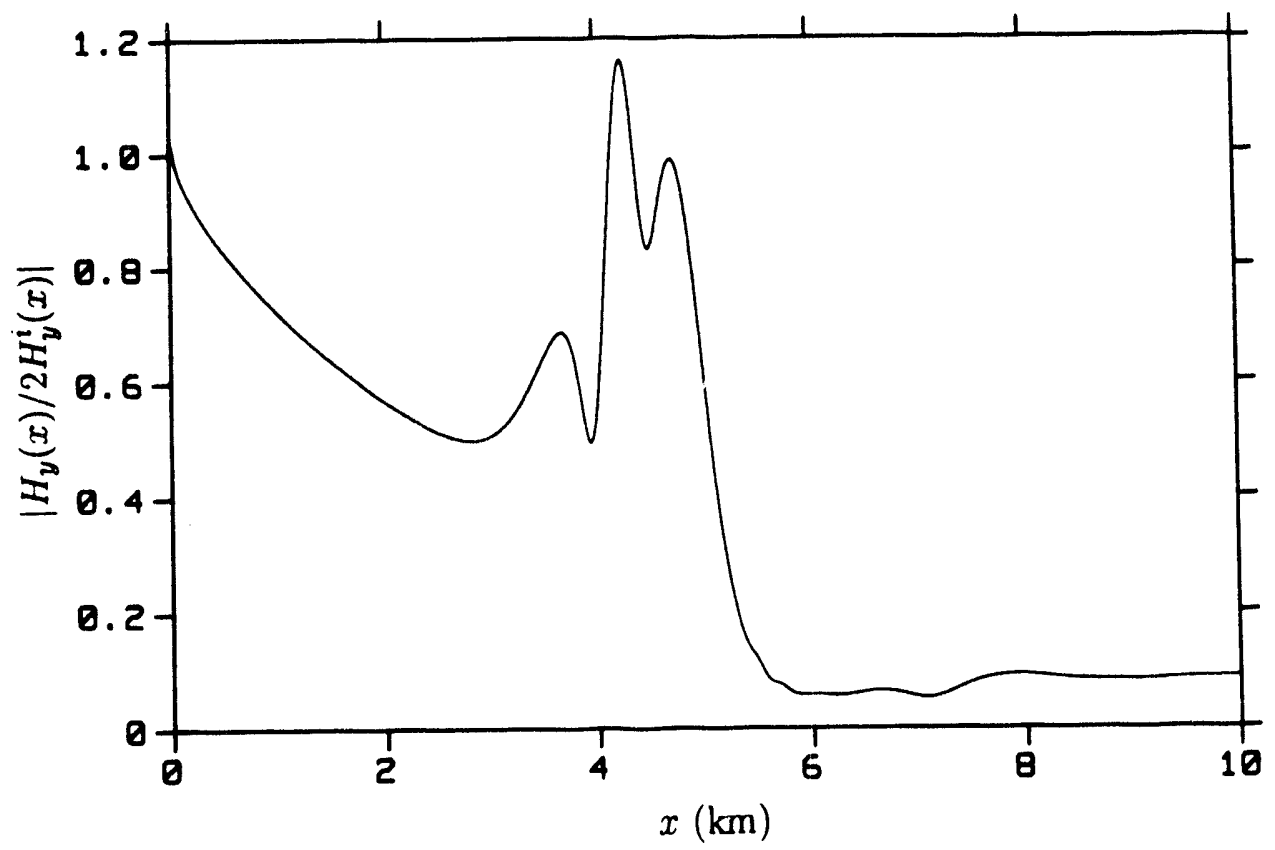

Fig. 8. Normalized $H_{y}$ over the Gaussian hill at $3 \mathrm{MHz}$ : a) result from program WAGNER; b) solution of integral equation (27).

with a smaller distance between source and scatterer. The results from 2-D PO and 3-D GO for the Gaussian hill at $10 \mathrm{MHz}$ are compared in Figure 12.

Most of the validation tests of the PO codes have made use of an actual terrain profile for which measured radiation patterns were available. The U.S. Navy is currently sponsoring a project to measure radiation patterns from antennas situated in irregular terrain as part of the effort to validate the model development reported here and by other workers $[7,16]$. 
a)

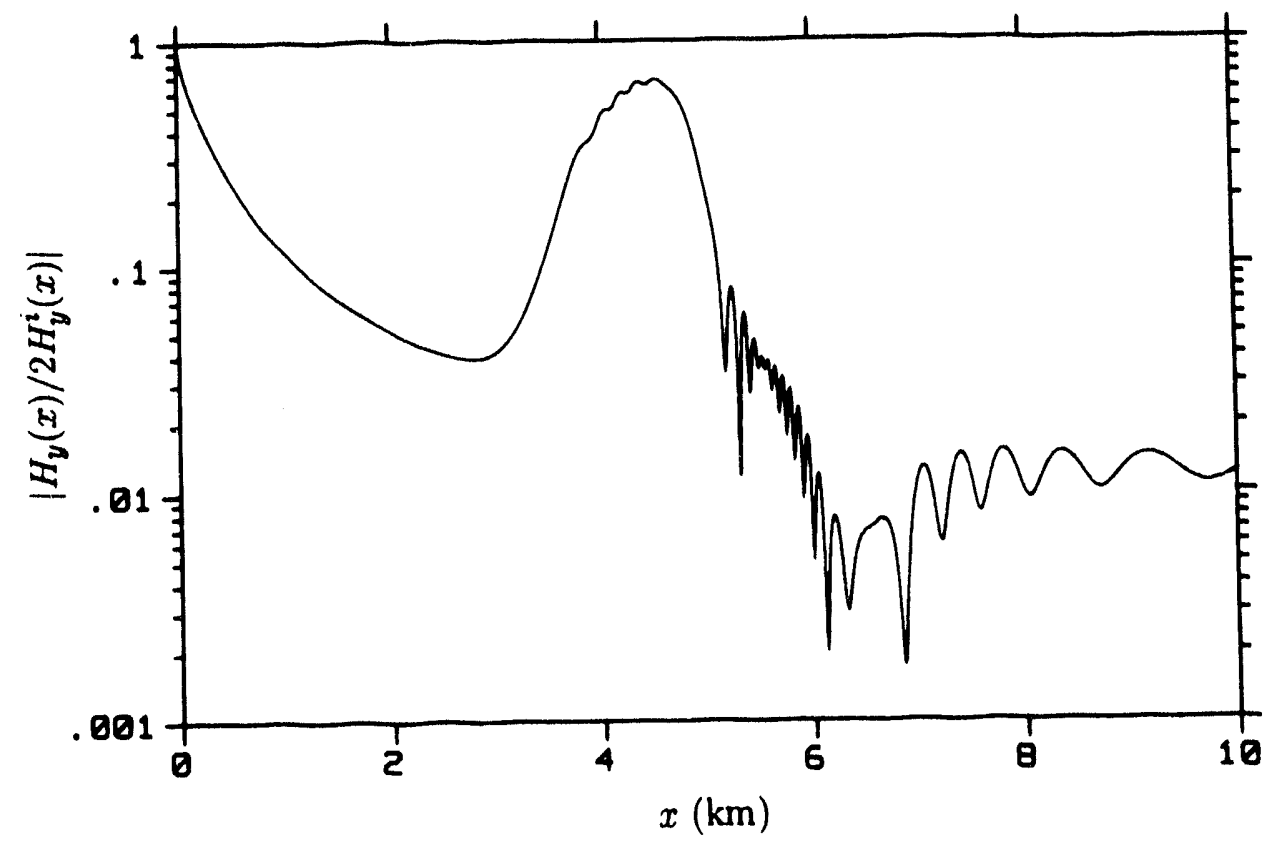

b)

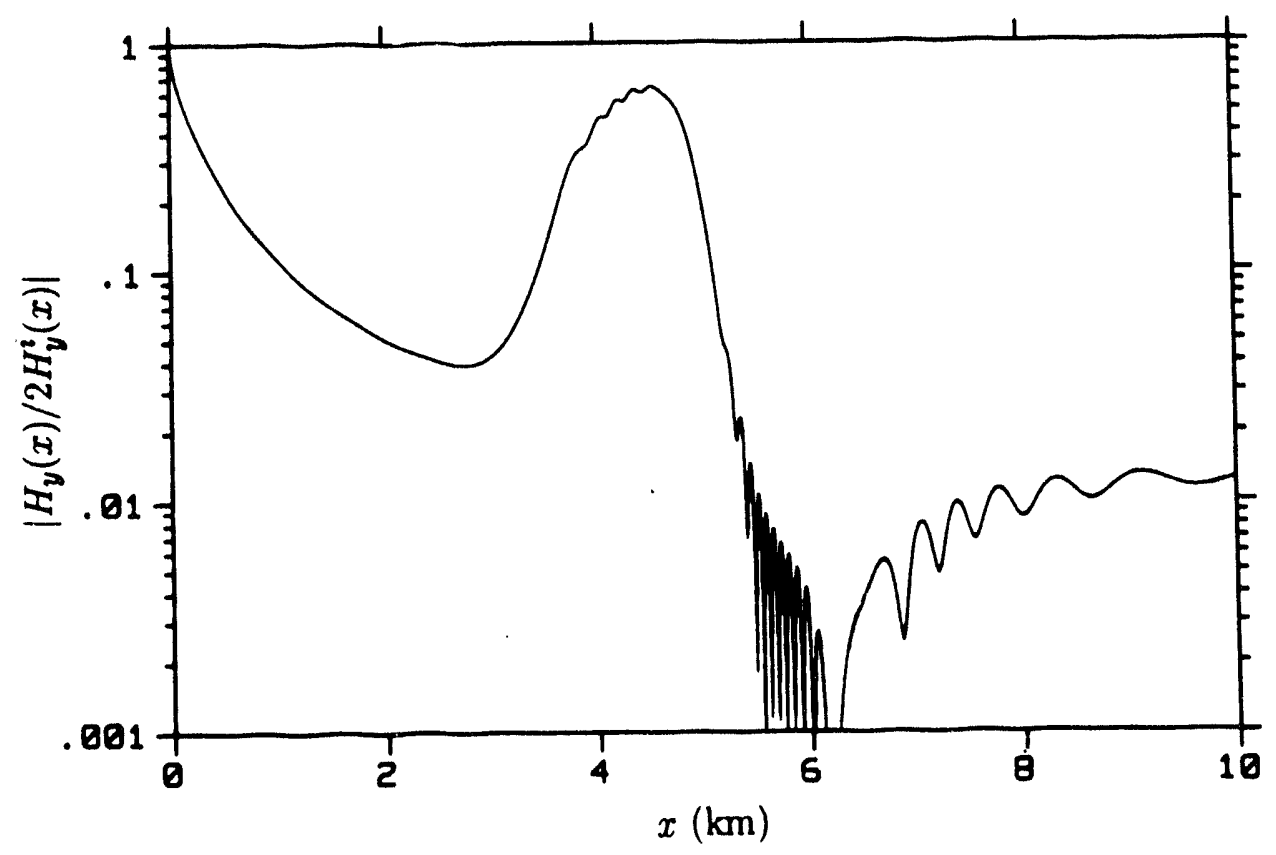

Fig. 9. Integral equation solution for the normalized $H_{y}$ over the Gaussian hill at $10 \mathrm{MHz}$ : a) result with 1400 unknowns; b) result with 3000 unknowns.

An initial set of measurements has been made at Cedar Valley in Utah [22], for which a topographic map is shown in Figure 13. Antennas were located at three sites indicated on the map as West Valley Monopole and Dipole, Hill Top Monopole and Dipole and East Valley Monopole and Dipole; and patterns were measured by a helicopter flying along the indicated route.

The PO and integral equation codes include interface routines to allow arbitrary terrain 


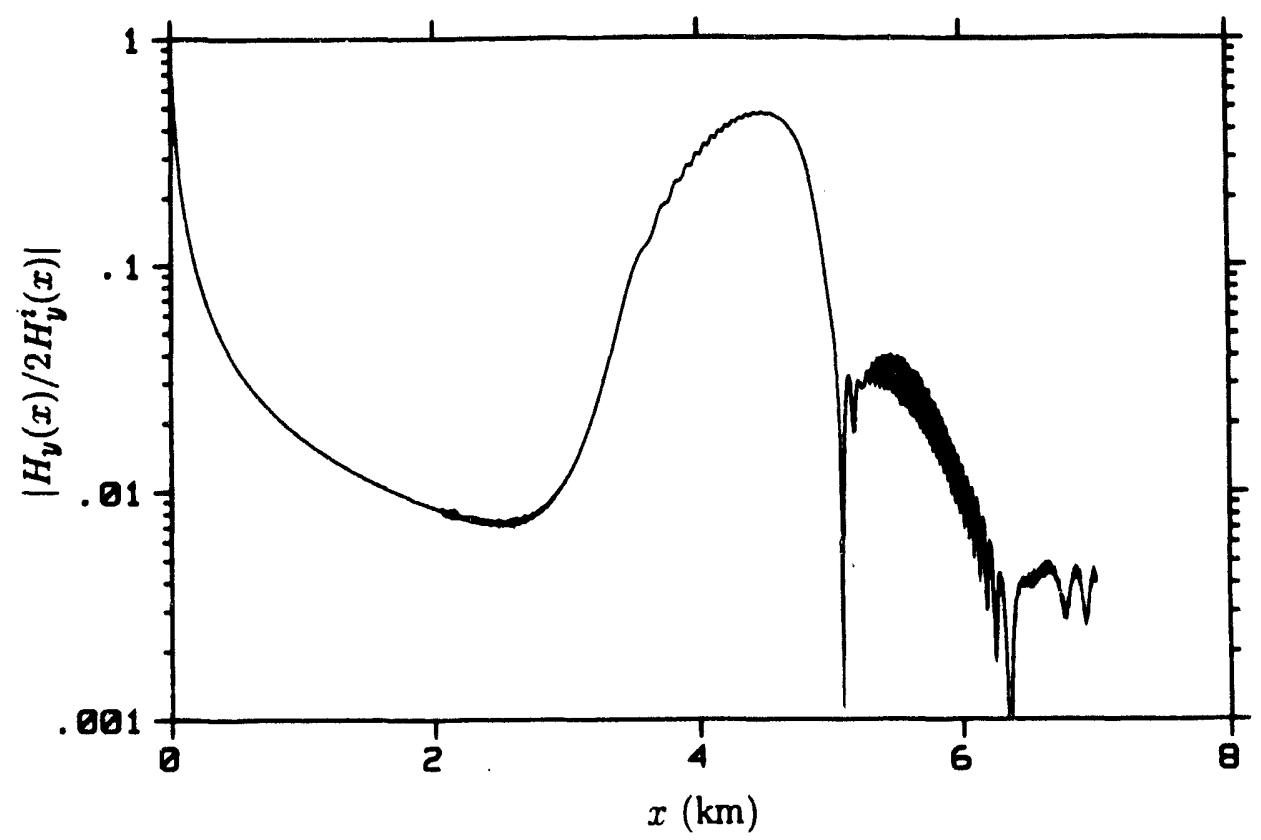

Fig. 10. Integral equation solution for the normalized $H_{y}$ over the Gaussian hill at $30 \mathrm{MHz}$ using 3000 unknowns over the range $-0.5 \mathrm{~km} \leq x \leq 7 \mathrm{~km}$.

profiles to be entered. For the 2-D models (2-D PO, integral equation and WAGNER) the terrain height is entered at points along the 2-D profile, and the code applies cubic-spline interpolation to obtain the heights at points needed in the solution. Spline interpolation was essential for WAGNER, since the solution became unstable with even small discontinuities in the surface derivative. For the Cedar Valley site the 2-D contour was taken along the helicopter flight path passing through Station 6, and $x=0$ was chosen adjacent to the West Valley antenna site. A reference elevation of 4990 feet on the topographic map was chosen as $z=0$. The hills actually continue to a large distance in positive $x$, but the numerical profile was brought to zero height for $x<-100 \mathrm{~m}$ and for $x>770 \mathrm{~m}$. With the terrain starting and ending at $z=0$ the integral equation code using images can be run, rather than truncating the current to infinity, when perfectly conducting ground is modeled. The interpolated surface profile for the Cedar Valley site is plotted in Figure 14a. The format of the terrain data file is described in Appendix B which also contains the data file that produced Figure 14a.

Figure 14b is a Gaussian curve chosen to approximate the height and front surface of the Cedar Valley profile. The Gaussian is defined by

$$
h_{s}(x)=45 \exp \left[-0.000114(x-170)^{2}\right] .
$$

Both the Gaussian and the Cedar Valley profiles have peaks at $x=170 \mathrm{~m}$. In the Cedar Valley profile the height at this peak is $50 \mathrm{~m}$ and the height at $x=0$ is $6 \mathrm{~m}$. For the Gaussian hill the peak at $x=170 \mathrm{~m}$ is $45 \mathrm{~m}$ and the height at $x=0$ is $1.65 \mathrm{~m}$. The Gaussian hill is the same as that used by Janaswamy in [16], and was included here to allow a comparison of the radiation patterns. In addition, the comparison of results from the Gaussian hill and Cedar Valley profiles can demonstrate the importance of small differences in a terrain profile 
a)

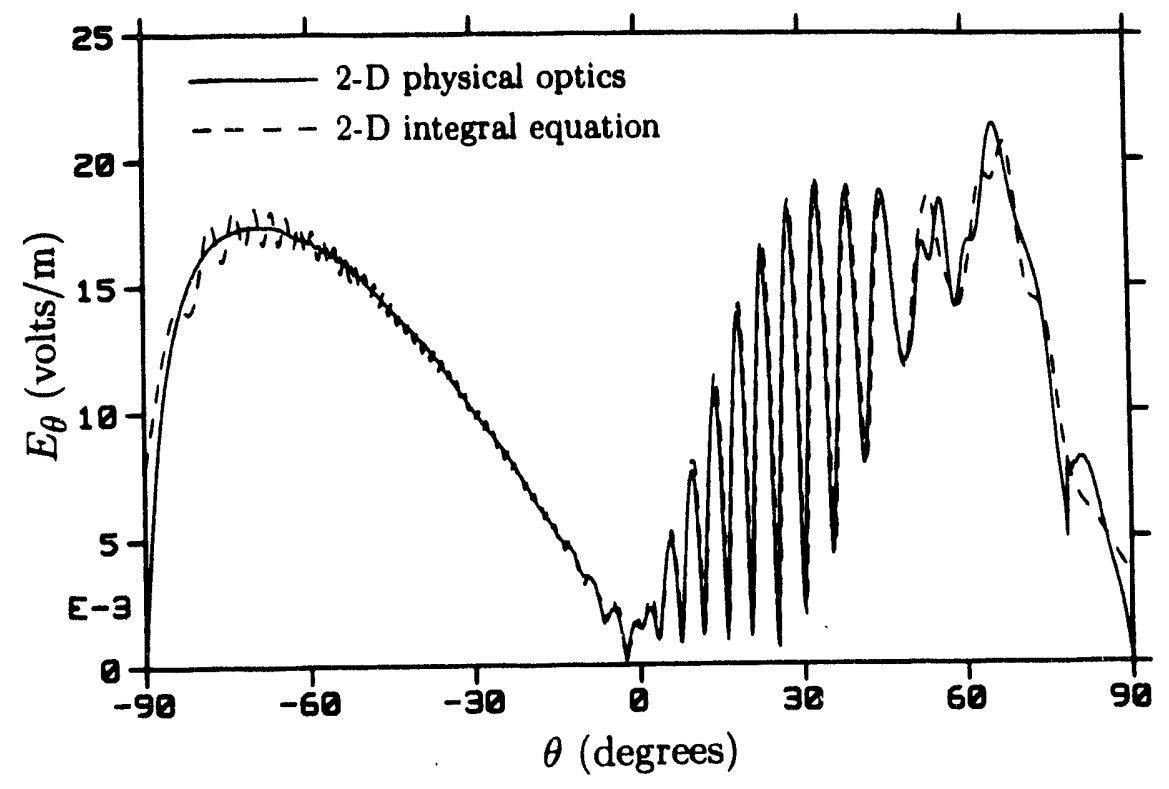

b)

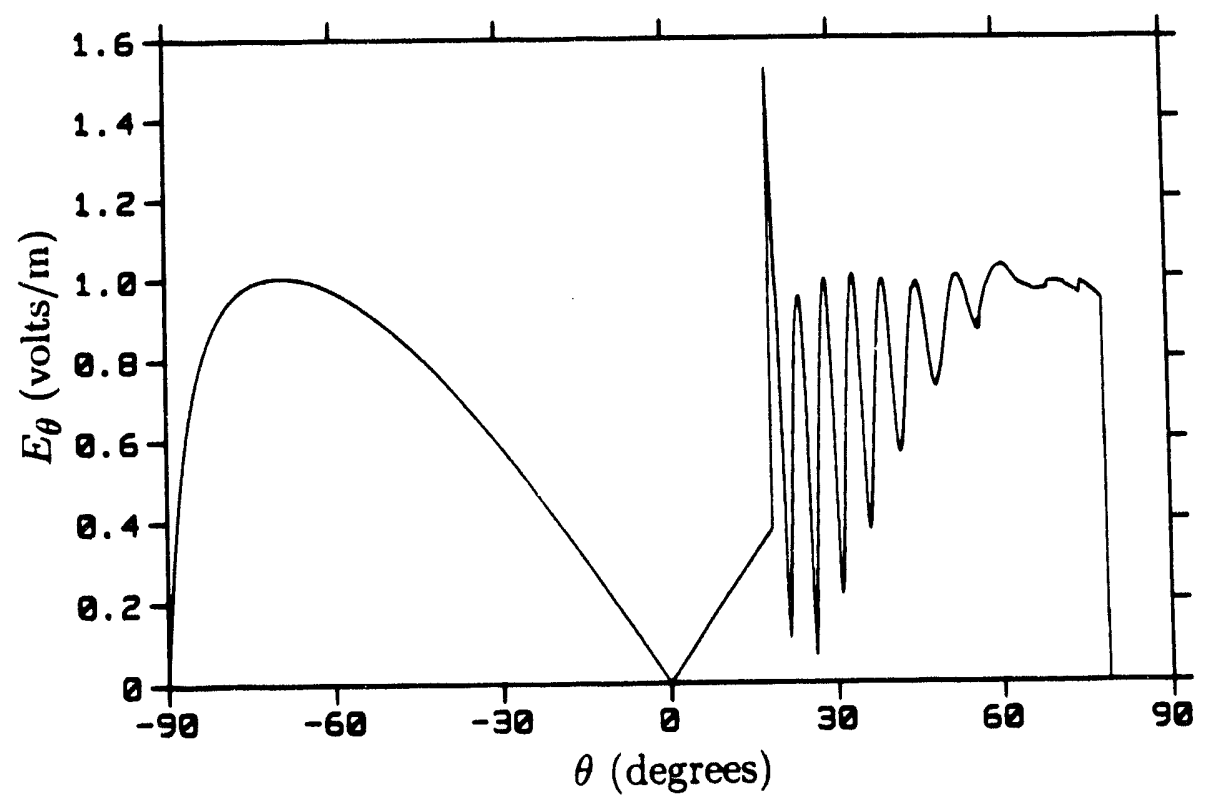

Fig. 11. Radiation patterns of a vertical dipole source $5 \mathrm{~km}$ from a Gaussian hill at $1 \mathrm{MHz}$, with ground parameters $\epsilon_{r}=10$ and $\sigma=0.01 \mathrm{~S} / \mathrm{m}$ : a) 2-D PO and integral equation solutions; b) 3-D geometrical optics result.

that might result from different readings of a map. Both curves probably differ considerably from the actual ground shape at Cedar Valley.

For the 3-D PO model a two dimensional spline interpolation was used to define the terrain surface. To generate the terrain surface description parallel lines are drawn on a topographic map in an arbitrary direction chosen as $x$. The spacing of these lines and of points along each line on the map are chosen to define the shape of important features of the terrain and the elevations at these points are entered into a file. The format of the data file is described in Appendix B. A surface plot obtained from spline interpolation of the points 
a)

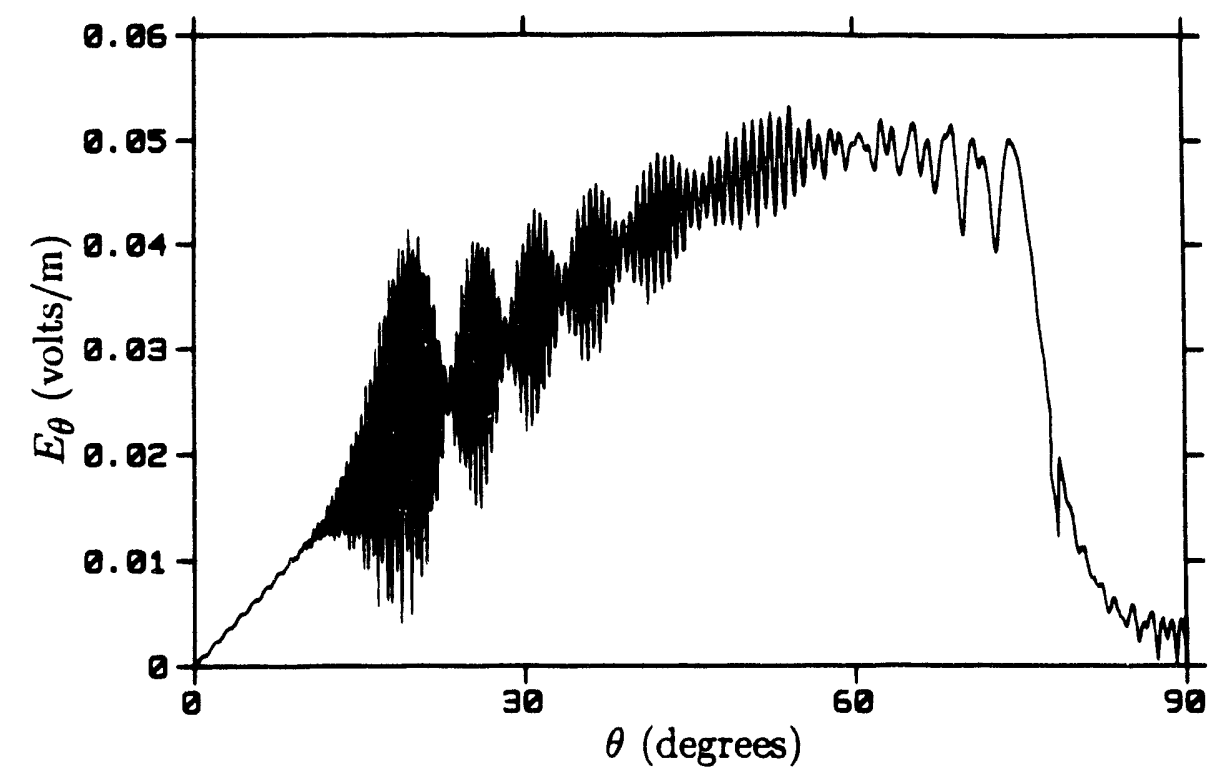

b)

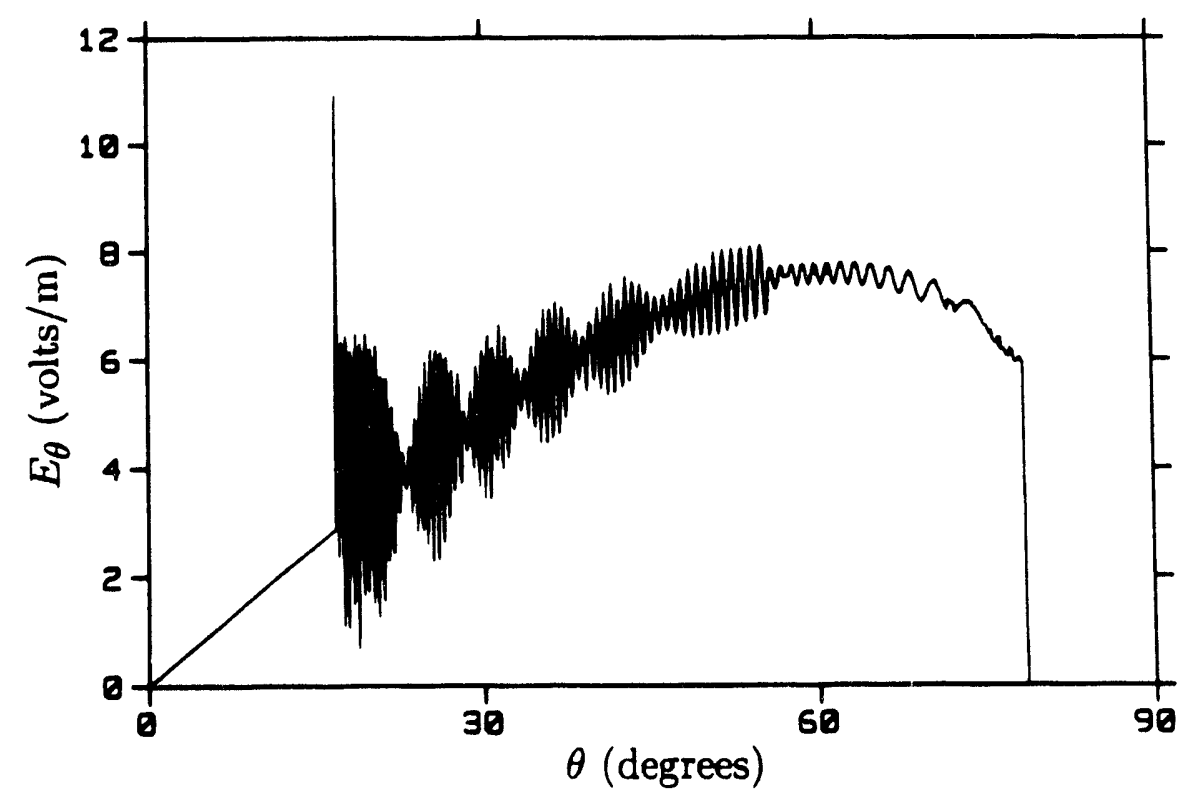

Fig. 12. Radiation patterns of a vertical dipole source $5 \mathrm{~km}$ from a Gaussian hill at $10 \mathrm{MHz}$ : a) 2-D PO result, b) 3-D geometrical optics solution.

read from the Cedar Valley map is shown in Figure 15. For the plot the vertical scale was multiplied by a factor of three to make the shape more visible.

Results from the integral equation solution and WAGNER for the normalized surface field $H_{y}$ over the 2-D Cedar Valley profile are shown in Figure 16 for a frequency of 8.015 $\mathrm{MHz}$ and in Figure 17 for $27.7415 \mathrm{MHz}$. The source was a strip of vertically directed electric current at a height of $1 \mathrm{~m}$ above the surface at $x=0$. These results are in reasonable agreement, and show the typical increase in strength on the near sides of peaks and decrease in the shadow regions. When PO is applied using the antenna at $x=0$ as the source, the PO surface current is zero beyond about $x=110 \mathrm{~m}$ due to blockage by the shoulder in the profile. 


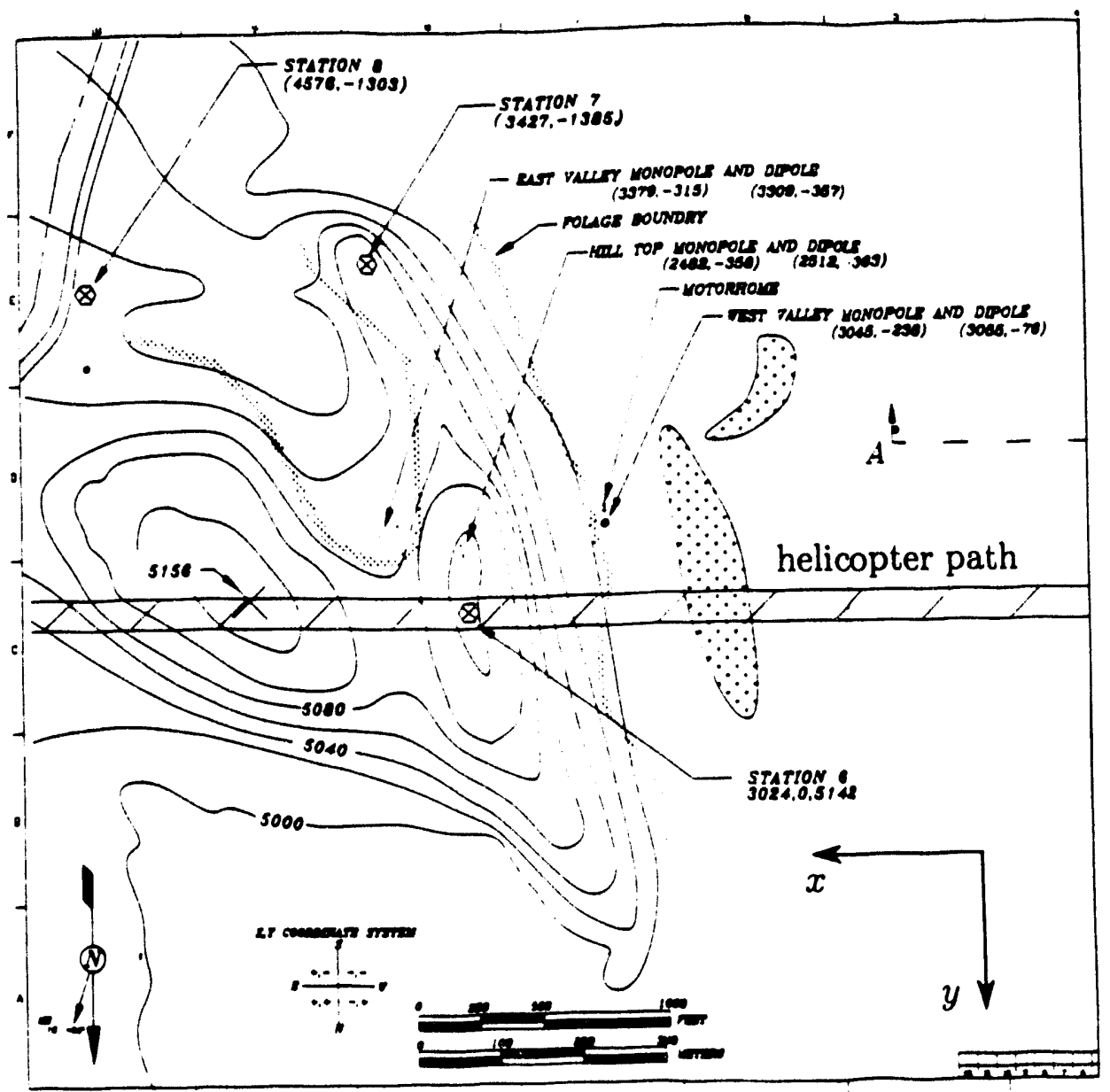

Fig. 13. Topographic map of the Cedar Valley Utah site.

a)

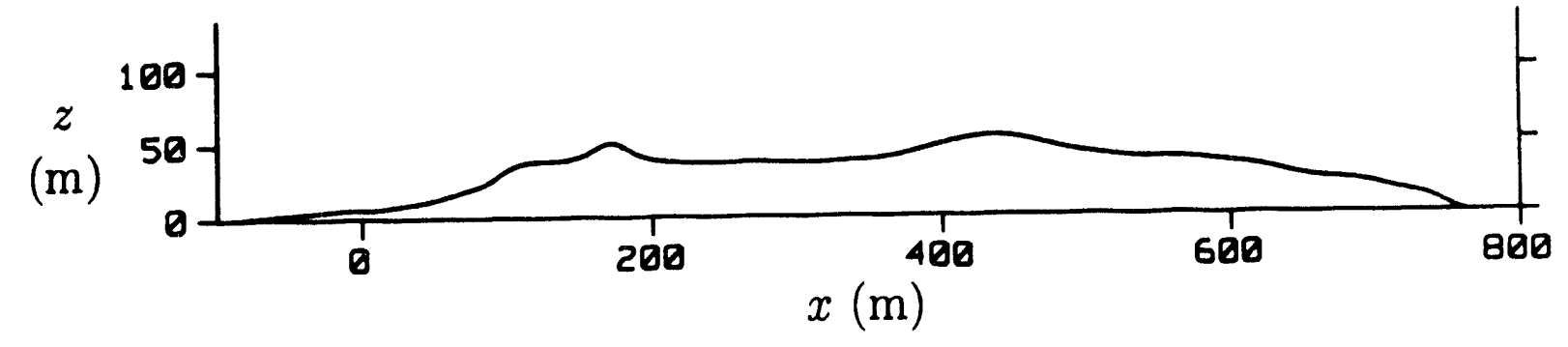

b)

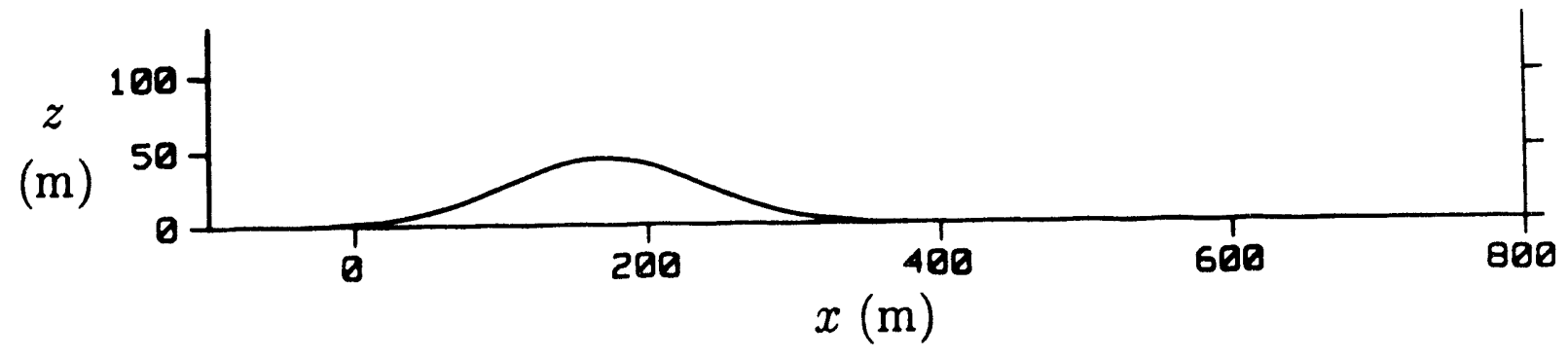

Fig. 14. Two-dimensional terrain profiles: a) Cedar Valley site along the helicopter flight path, b) Gaussian curve approximating the front side of the hill at Cedar Valley. 


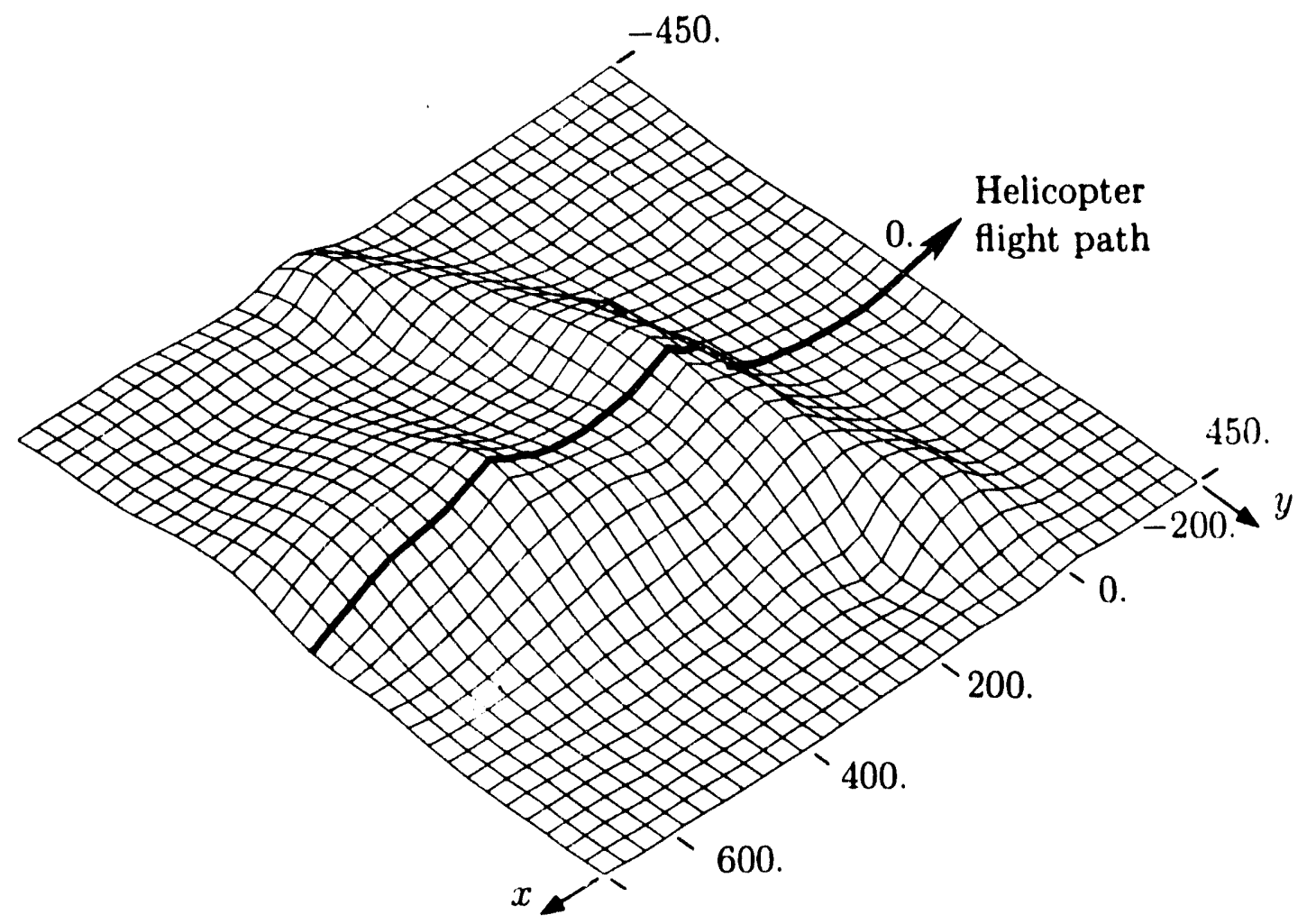

Fig. 15. Surface plot from cubic-spline interpolation of elevation points read from the Cedar Valley topographic map. Coordinates are in meters and the vertical scale has been multiplied by a factor of 3 .

of Figure 14a. It can be seen from Figures 16 and 17 that this is not a good approximation to the current, although it is not as bad as might appear since the unnormalized surface field decreases faster than the results shown in the figures. A few comparisons of PO and integral equation currents with reciprocity invoked to put the source at the distant receiver location, as in the PO codes developed here, have shown good agreement. Over illuminated regions the currents almost coincide, while at shadow boundaries the discontinuities in the PO current are replaced by continuous but rapid changes in the integral equation currents.

Radiation pattern measurements were made at Cedar Valley for antennas located at the West Valley, Hill Top and East Valley sites [22]. Two antennas were measured at each site: a vertical monopole with height of $16 \mathrm{ft}$ and a horizontal $\lambda / 2$ dipole (adjusted for each frequency) at a height of $15 \mathrm{ft}$ and oriented normal to the helicopter flight path. Measurements were made at frequencies of 8.015 and $27.7415 \mathrm{MHz}$. Also, $15.3415 \mathrm{MHz}$ and a frequency around $50 \mathrm{MHz}$ were used, but not all of this data is presently available. The ground parameters were measured as $\epsilon_{r}=14.9$ and $\sigma=0.0065 \mathrm{~S} / \mathrm{m}$ at $8.015 \mathrm{MHz}$, and $\epsilon_{r}=9.5$ and $\sigma=0.0161 \mathrm{~S} / \mathrm{m}$ at $27.7415 \mathrm{MHz}$.

For validating the $\mathrm{PO}$ model, the monopole and horizontal dipole antennas located at the West Valley and Hill Top sites were modeled at 8.015 and $27.7415 \mathrm{MHz}$. Radiation patterns from the PO code were first compared with computed patterns from the integral equation code to postpone dealing with the real-world uncertainties of the measurements. 
a)

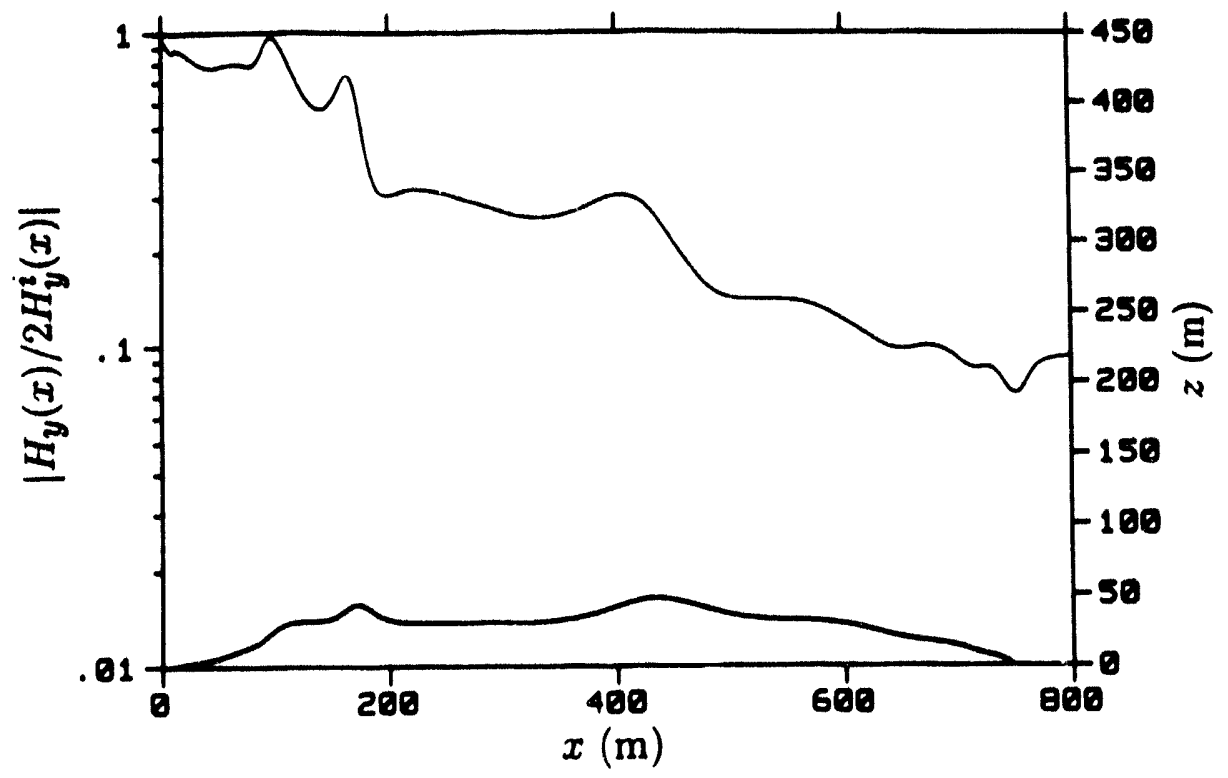

b)

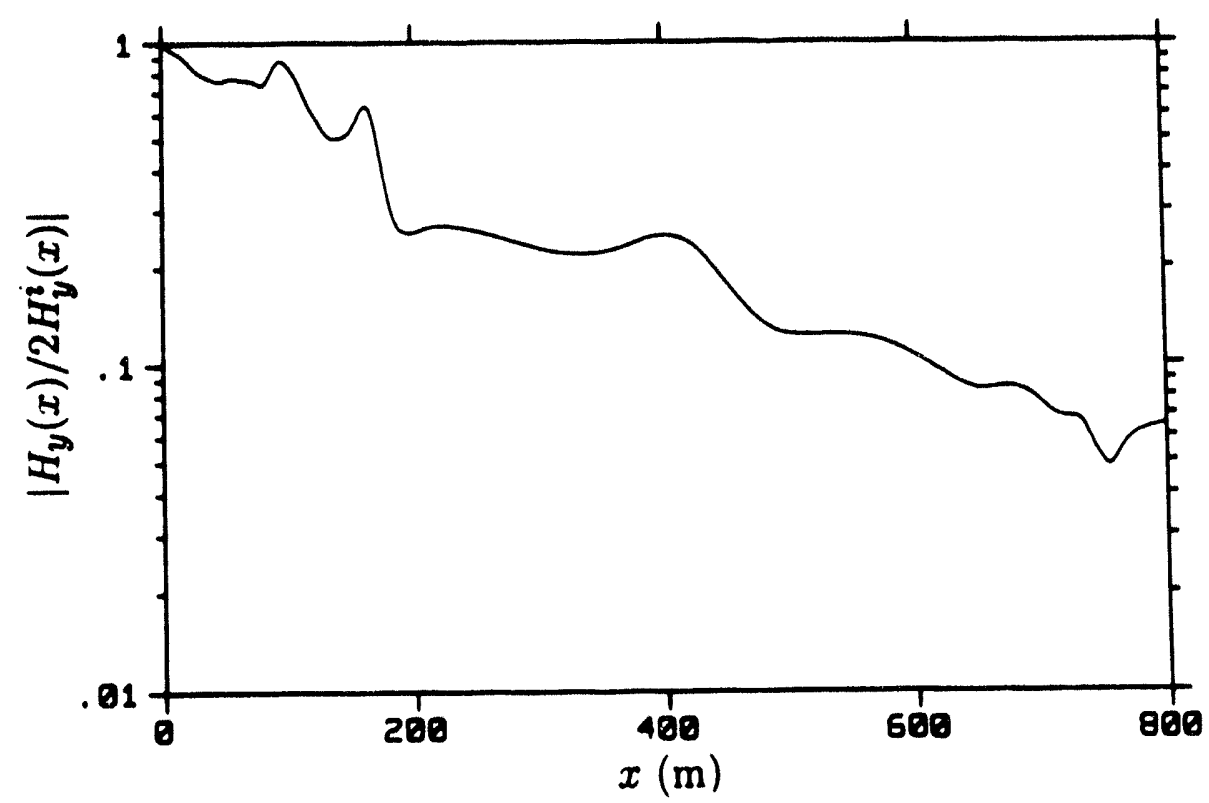

Fig. 16. Normalized field $H_{y}$ over the 2-D Cedar Valley profile at $8.015 \mathrm{MHz}$ : a) result from program WAGNER, b) integral equation solution.

Since the integral equation codes will only treat a single line source with vertical or horizontal polarization, the vertical monopole was represented as a vertical strip of electric current at a height of $2 \mathrm{~m}$, infinite in the $y$ direction and with $I_{z} d z=1$ Ampere-meter, while the horizontal dipole was represented by a horizontal electric line source with a current $I_{y} d z=1$ Ampere-meter at a height of $4.57 \mathrm{~m}$. When the PO code was run using a NEC solution for the actual current on a $16 \mathrm{ft}$ monopole and a $\lambda / 2$ dipole the patterns were very close to those obtained with point sources, so the point-source results will also be compared with the measurements. 
a)

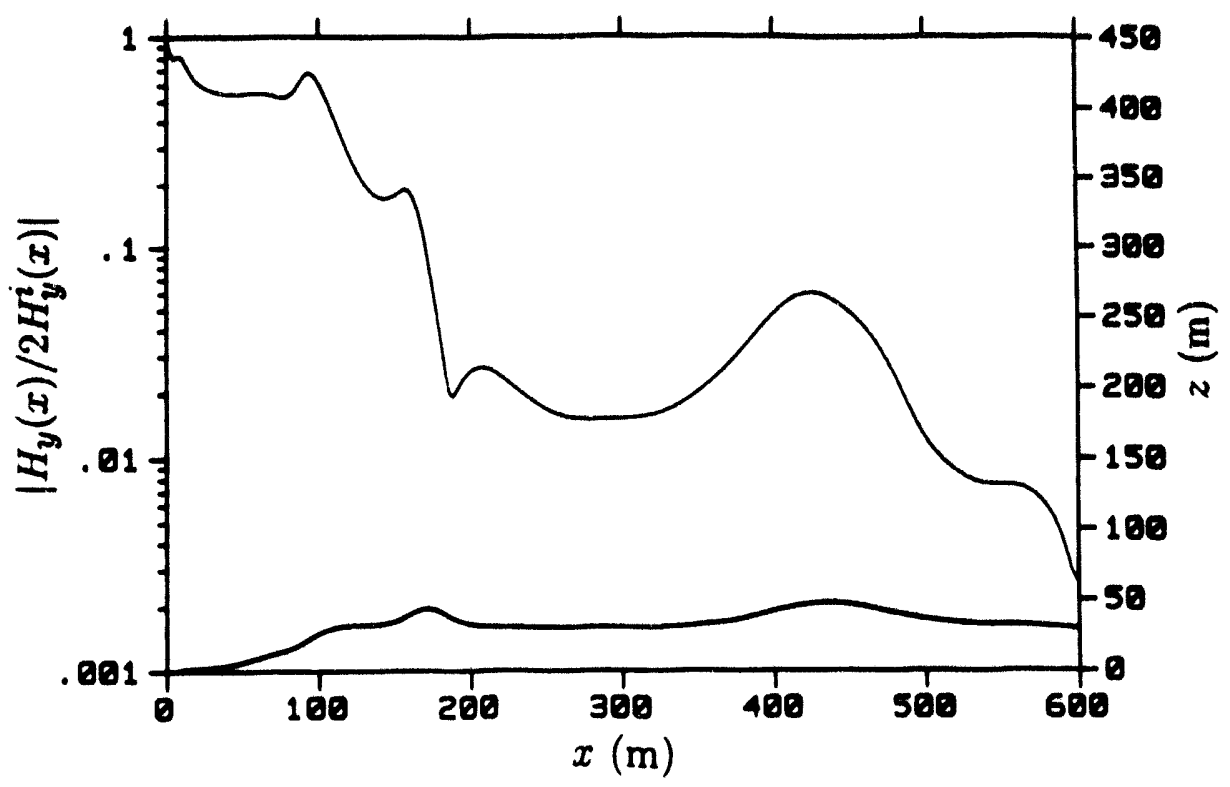

b)

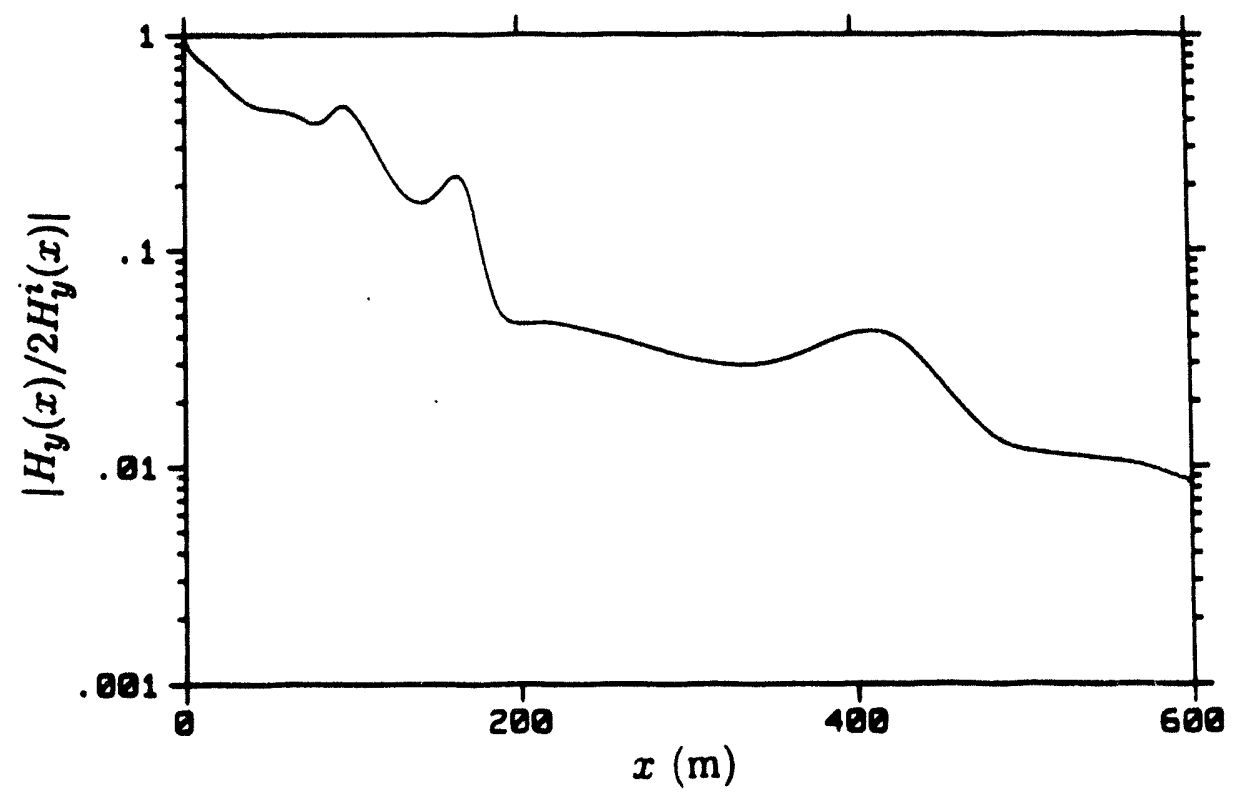

Fig. 17. Normalized field $H_{y}$ over the 2-D Cedar Valley profile at $27.7415 \mathrm{MHz}$ : a) result from program WAGNER, b) integral equation solution.

The comparisons of the $\mathrm{PO}$ and integral equation solutions are shown in Figures 18 through 25. Each figure includes patterns for the antenna at corresponding locations on the Cedar Valley profile of Figure 14a and on the simple Gaussian hill of Figure 14b. In addition, patterns are shown for the actual ground parameters and perfectly conducting ground. Perfectly conducting ground is of interest for two reasons. The integral equation code using images was used to model perfectly conducting ground, so there will be no distortions of the pattern due to truncating the current on the surface to infinity. Also, one of the questions about the PO model is the accuracy of using a reflection coefficient for an average angle of incidence in determining the second-order reflected field at $\left(x_{a}^{\prime}, z_{a}^{\prime}\right)$. For 

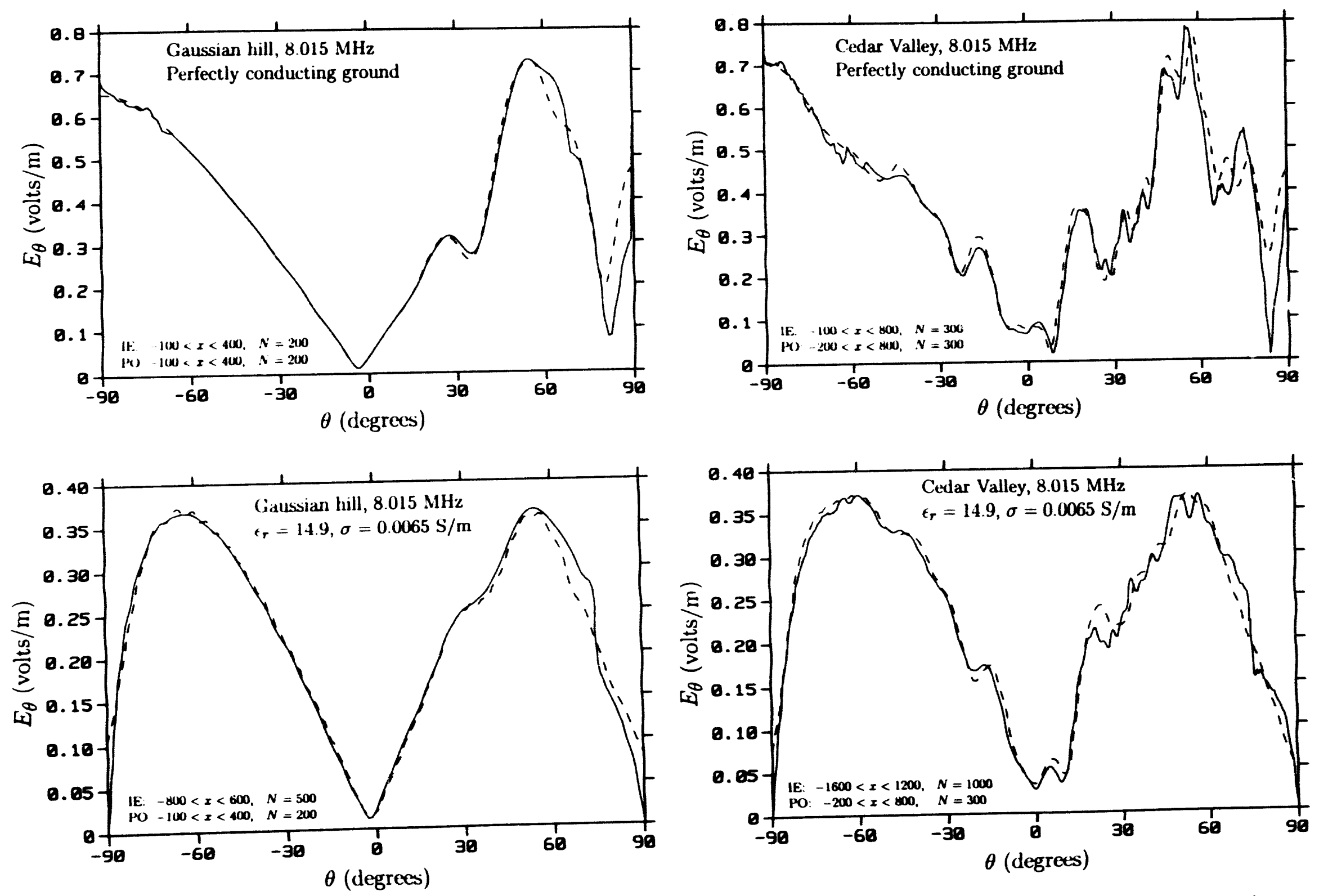

作 Fig. 18. Radiation patterns of a vertical dipole in front of the hill $\left(x_{a}=0, z_{a}=\right.$ surface +2 and integral equation solution $(--+-)$ are compared. 

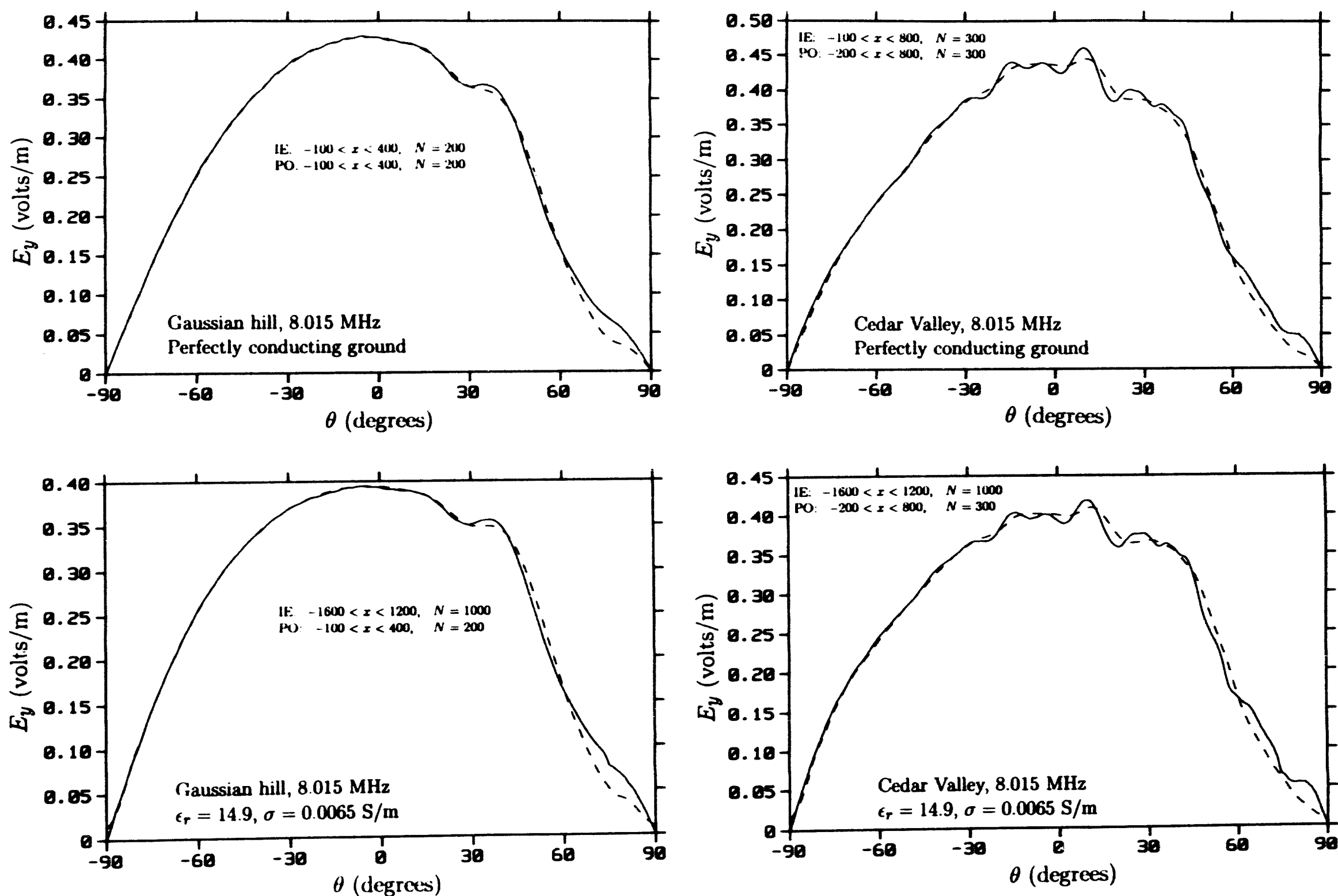

19. Radiation patterns of a horizontal dipole in front of the hill $\left(x_{a}=0, z_{a}=\right.$ surface $\left.+4.57 \mathrm{~m}\right)$ for the Gaussain hill and Cedar Valley terrain at $8.015 \mathrm{MIlz}$. Results from the $\mathrm{PO}$ ) model (___ ) and integral equation solution (_- - $)$ are compared. 


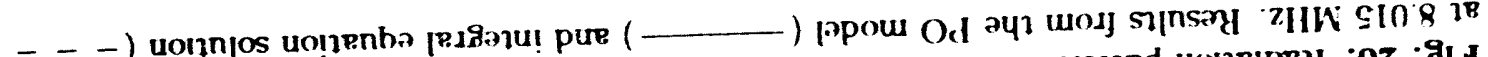

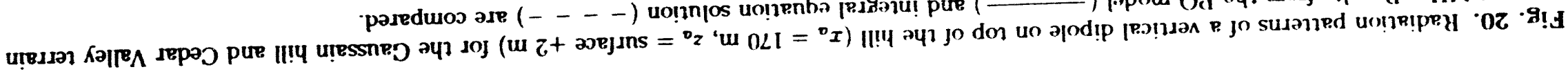
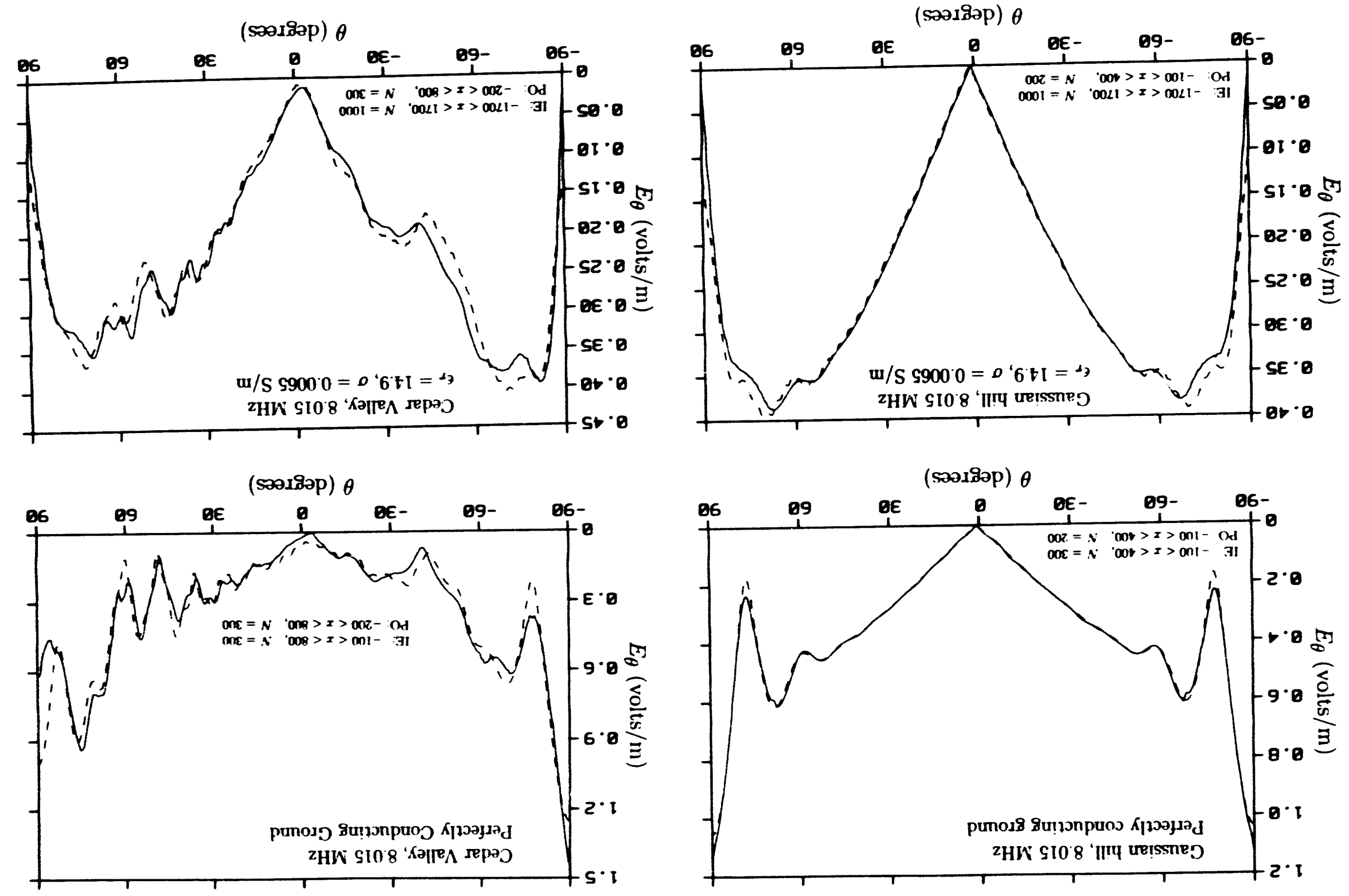

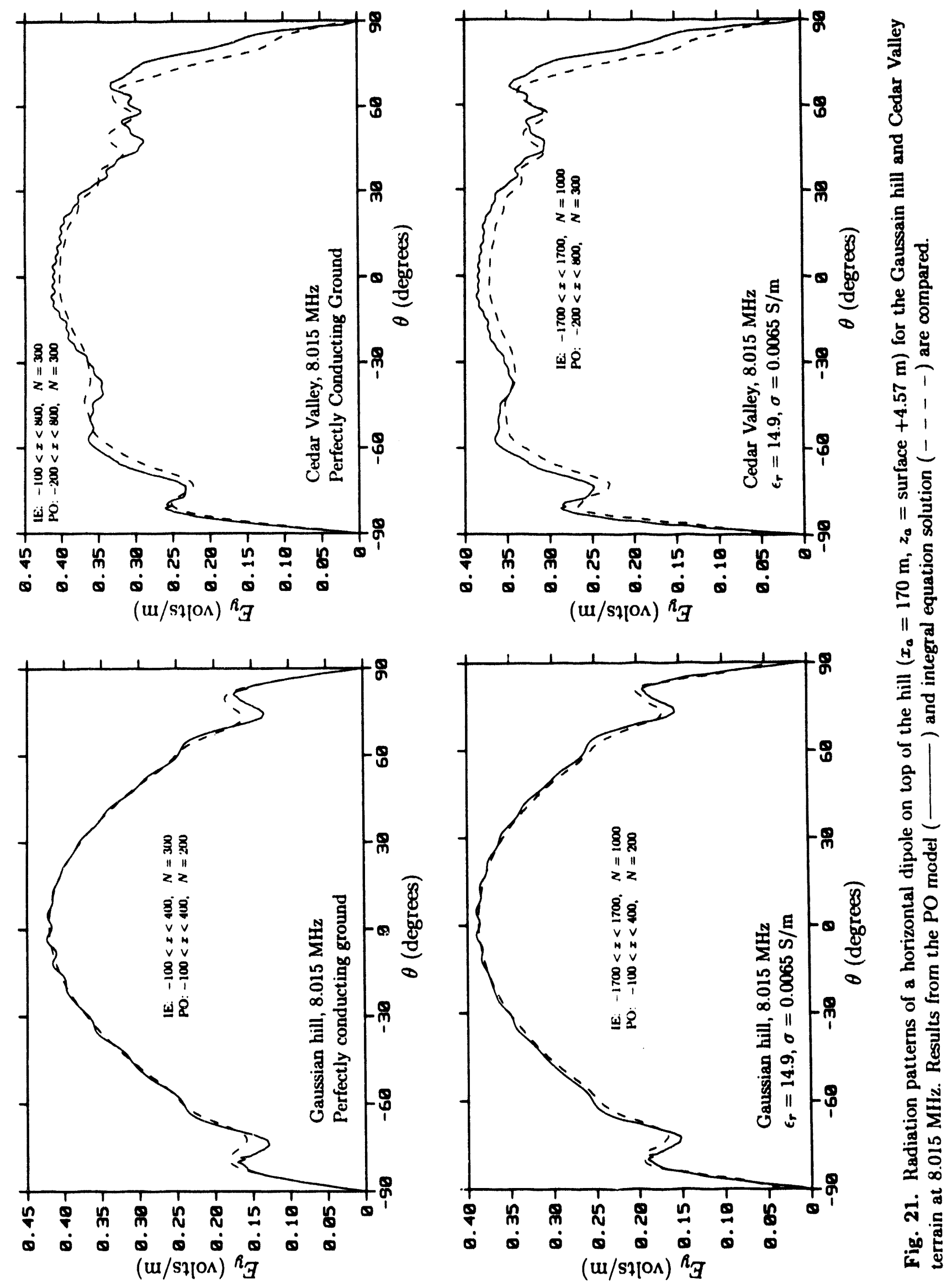

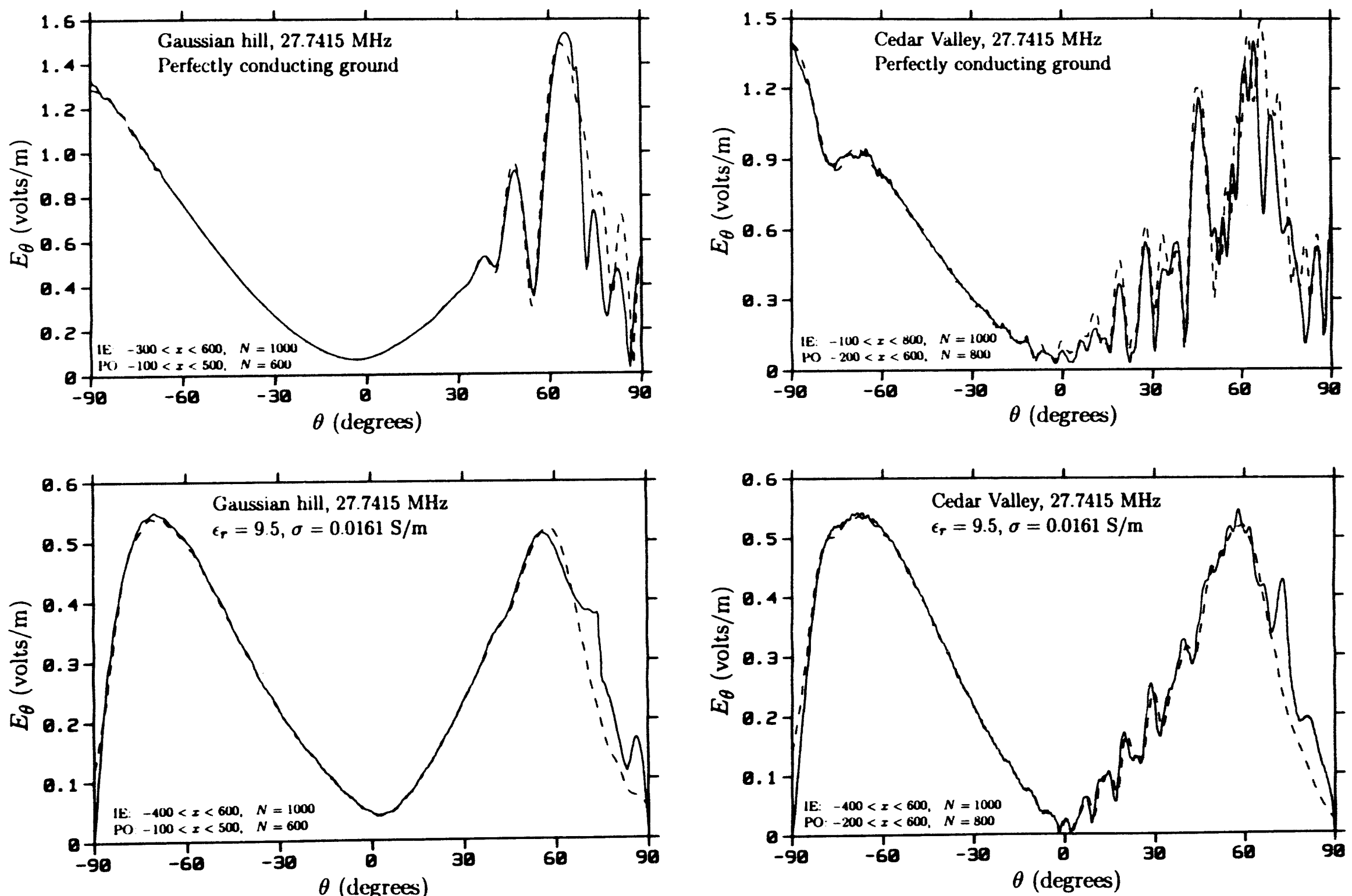

Fig. 22. Radiation patterns of a vertical dipole in front of the hill $\left(x_{a}=0, z_{a}=\right.$ surface $\left.+2 \mathrm{~m}\right)$ for the Gaussain hill and Cedar Valley terrain at 27.7415 MIlz. Results frorn the PO model ( ) and integral equation solution (- - - ) are compared. 

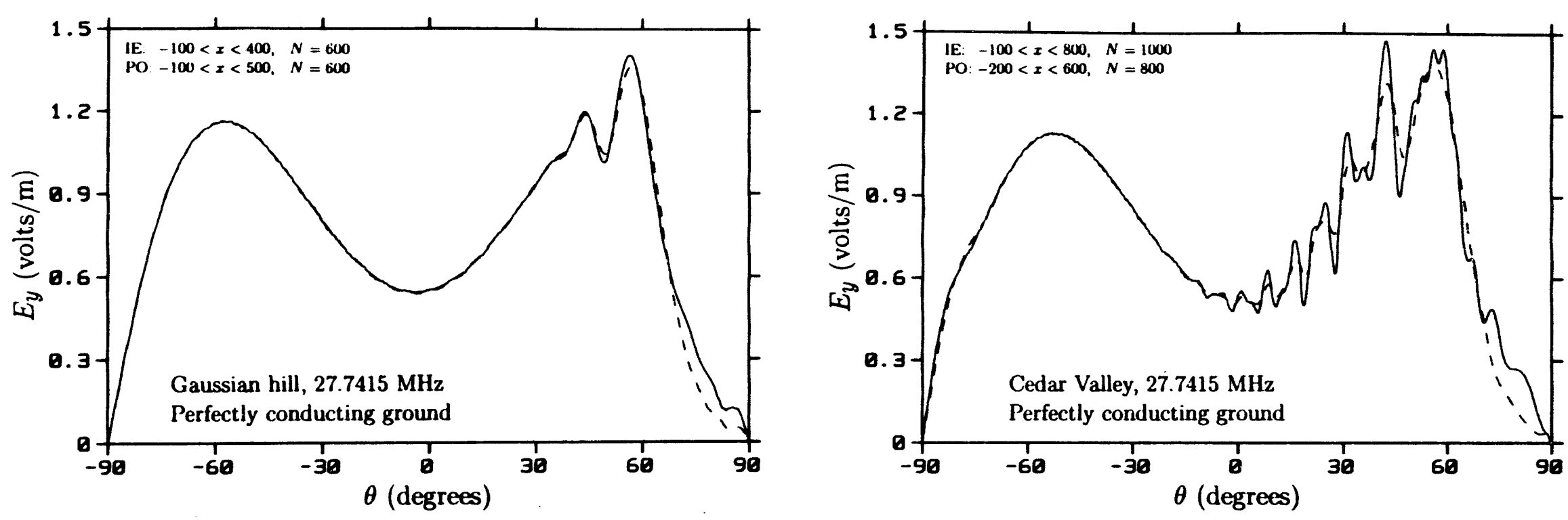

$\mathscr{L}$
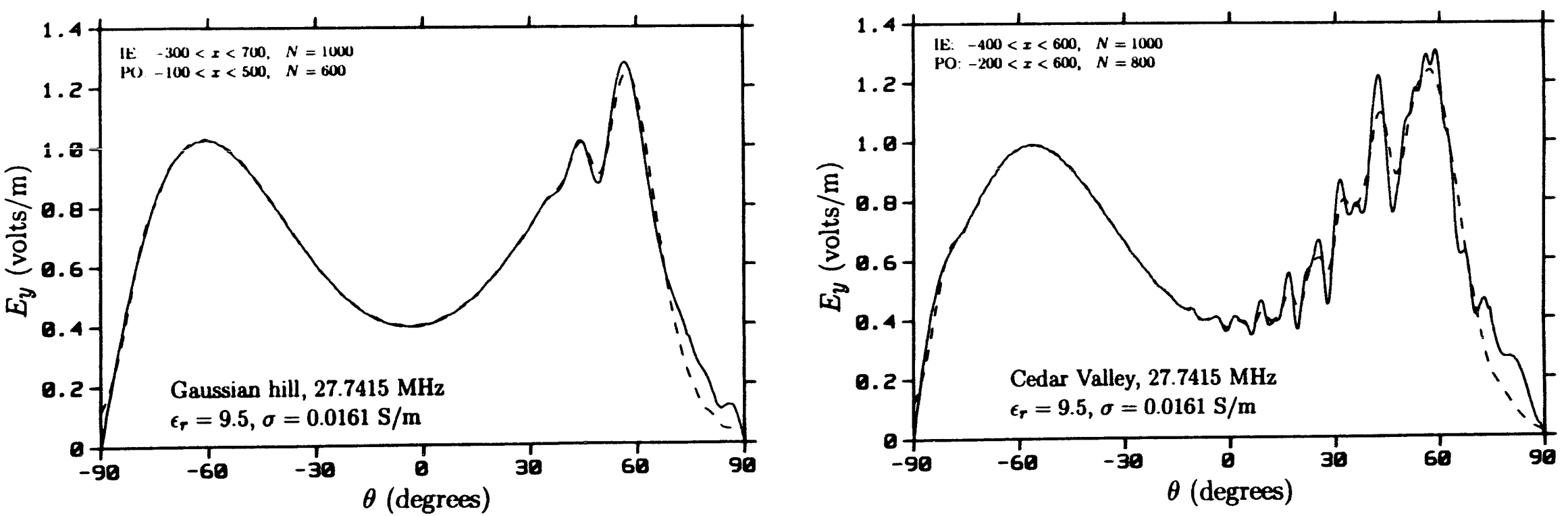

Fig. 23. Radiation patterns of a horizontal dipole in front of the hill $\left(x_{a}=0, z_{a}=\right.$ surface $\left.+4.57 \mathrm{~m}\right)$ for the Gaussain hill and Cedar Valley terrain at $27.7415 \mathrm{MHz}$. Results from the PO model ( 

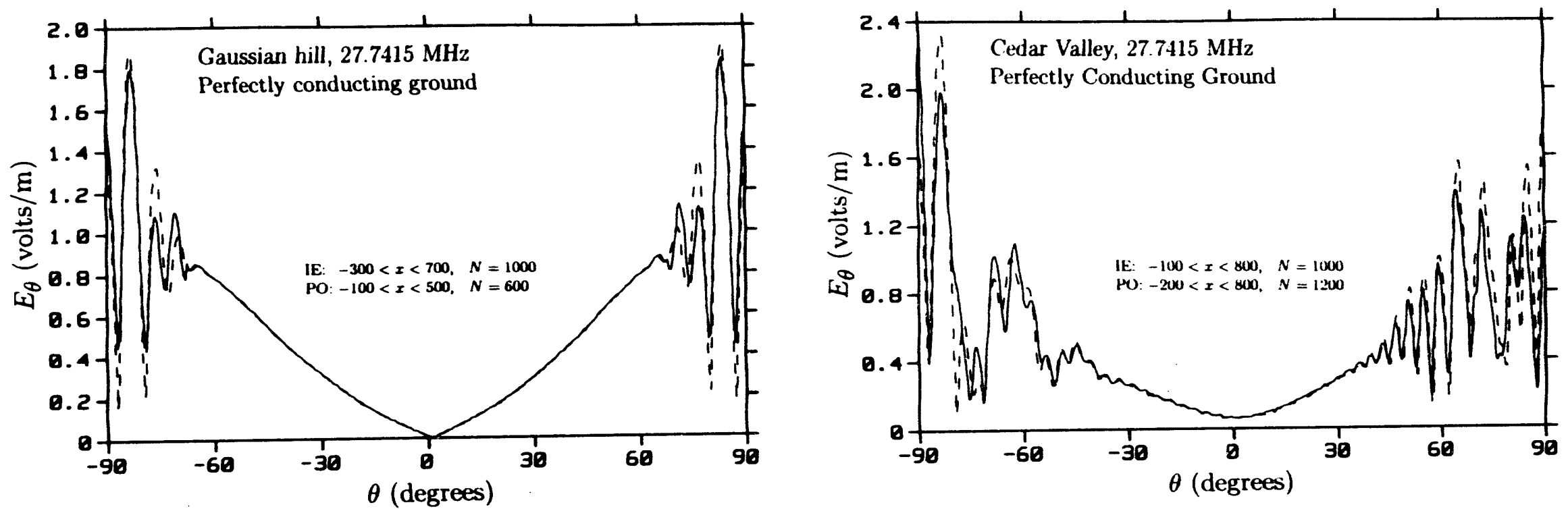

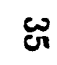
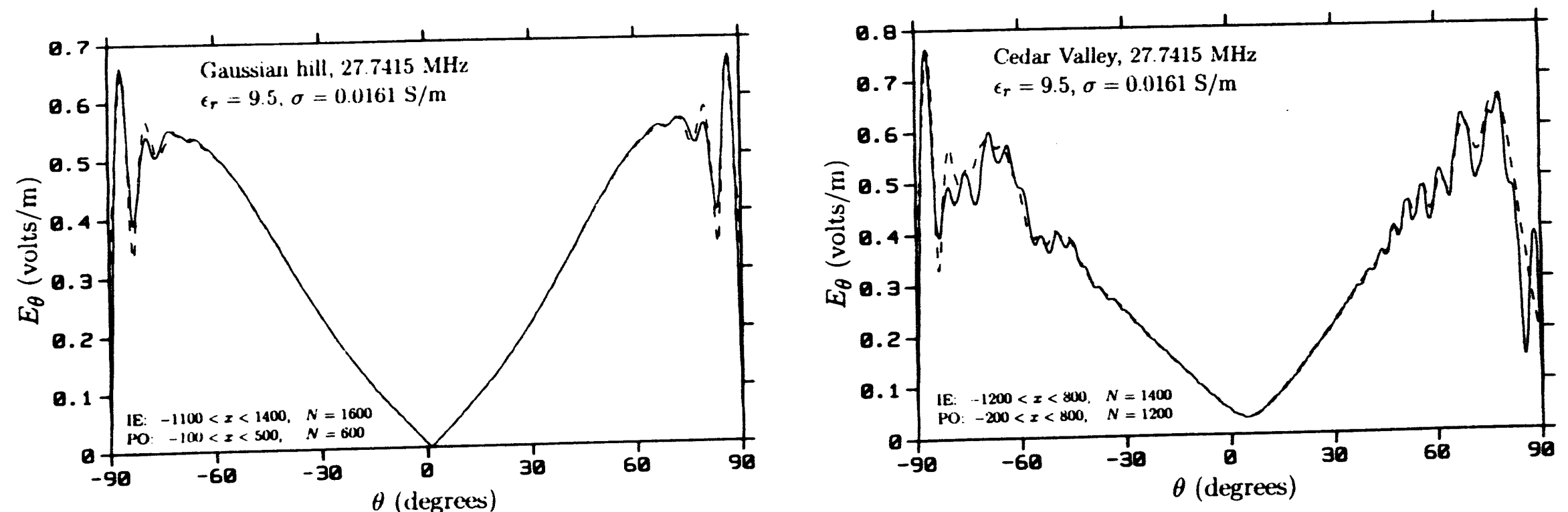

Fig Radiation patterns of a vertical dipole on top of the hill $\left(x_{a}=170 \mathrm{~m}, z_{a}=\right.$ surface $\left.+2 \mathrm{~m}\right)$ for the Gaussain hill and Cedar Valley terrain Fig. 24. Radiation patterns of a vertical dipole on top of the hill $\left(x_{a}=170 \mathrm{~m}, z_{a}=\right.$ surface $\left.+2 \mathrm{~m}\right)$ and integral equation solution (- - - are compared. 

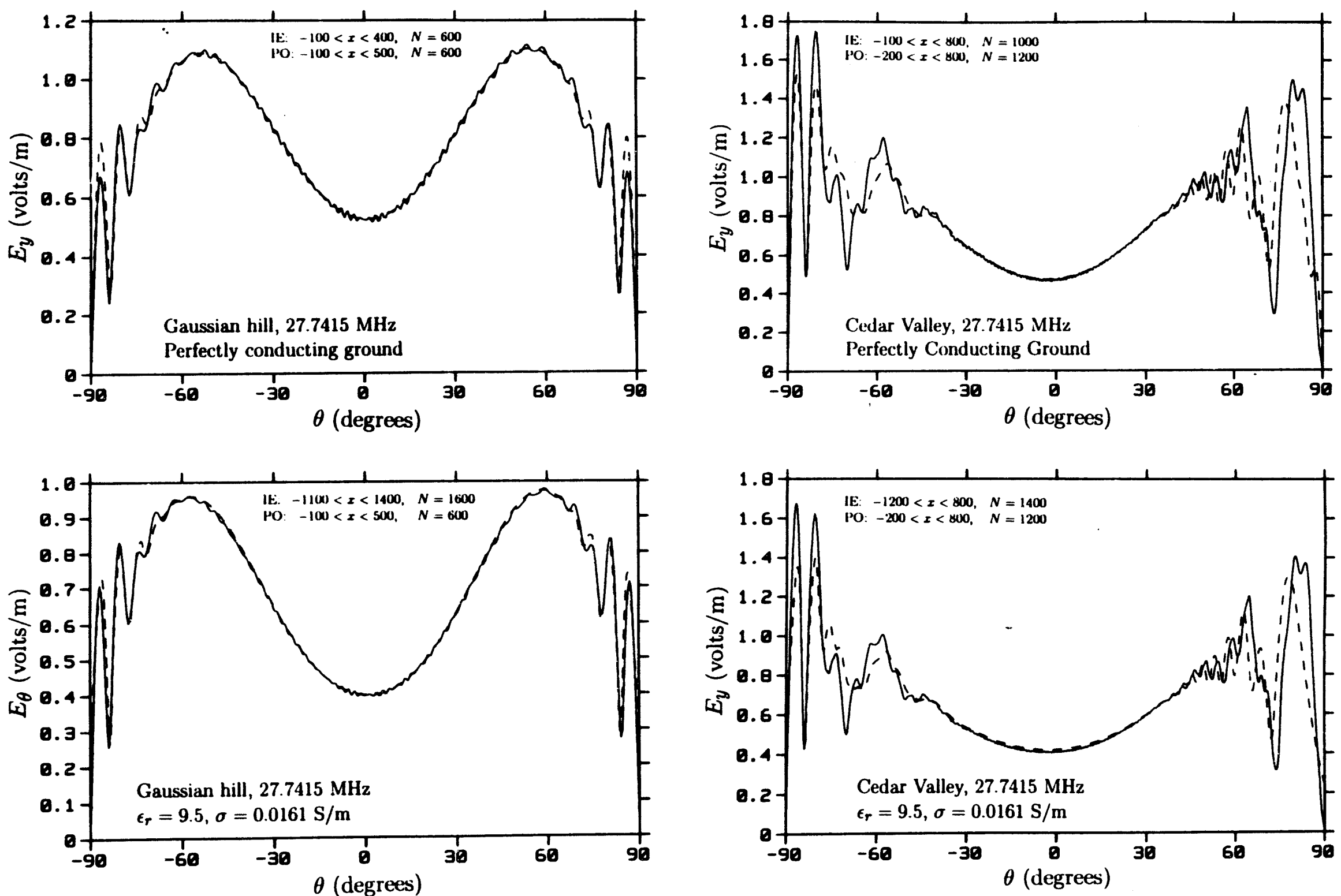

Fig. 25. Radiation patterns of a horizontal dipole on top of the hill $\left(x_{a}=170 \mathrm{~m}, z_{a}=\right.$ surface $\left.+4.57 \mathrm{~m}\right)$ for the Gaussain hill and Cedar Valley terrain at $27.7415 \mathrm{MHz}$. Results from the PO model ( ) and integral equation solution (- - - ) are compared. 
perfectly conducting ground the reflection coefficient is independent of incidence angle, so this source of error is eliminated. However, the approximation that the field reflects in a plane tangent to the ground under the antenna remains in question.

The patterns were computed with the receiving antenna (source in PO) sweeping an arc with radius $10^{4} \mathrm{~m}$ and positive $\theta$ is toward the hills, in the direction of positive $x$ in Figure 14. All of the results show generally good agreement between the PO and integral equation solutions. The most noticeable discrepancy occurs in the results for a vertical source in front of the hill for $\theta$ around 75 degrees. The rapid drop in the PO result in this case seems to be associated with the shadow boundary passing under the antenna on the ground. It might be possible to reduce this error by applying a smoothing window to the current at the shadow boundary.

The 2D PO results are compared with the measured patterns from [22] in Figures 26 through 29. It should be noted that these patterns have been reversed from left to right as compared to those in Figures 18 through 25 so that the patterns can be compared directly with those in [7]. As expected, the agreement between PO and measurements is not as good as that between $\mathrm{PO}$ and integral equation solutions. Most of these differences are probably due to factors in the measurement situation that have not been taken into account in the model. For example, the measured pattern for the monopole in front of the hill at 27.7415 $\mathrm{MHz}$, in Figure 26, shows a distinct flattening of the monopole pattern in the direction away from the hill for elevation angles from 10 to 60 degrees. Two separate measurements, with the helicopter carrying a vertical dipole and a horizontal dipole in the $x$ direction, show this effect. However, the PO and integral equation solutions and the UTD results from [7] all show almost no distortion of the monopole pattern in this region. Given the uncertainties in the measurement situation and terrain shape, the agreement between PO and measurements seems reasonably good.

\section{CONCLUSION}

The method of physical optics (PO) was used to develop numerical models for the scattering of antenna radiation by irregular terrain. Both 2D and 3D models were developed, since the 2D model offers a faster solution and simpler definition of the terrain shape, while the 3D model is needed when scattering occurs from obstacles out of the vertical plane from transmitter to receiver. The codes were designed to compute the skywave radiation patterns of antennas located on the ground. However, to get an accurate solution through PO it was necessary to invoke reciprocity to put the source at the distant receiver location and evaluate the field at the antenna location on the ground. The first-order PO solution was combined with image evaluations at the source and evaluation points to account for secondorder reflections. Also, a UTD solution for a half-plane with impedance boundary condition was included to extend the ground plane from the edges of the PO integration to infinity.

A 2D integral equation code was developed to validate the PO results. The EFIE was solved for horizontal polarization and the MFIE for vertical polarization with an impedance boundary condition on the surface. The equations were solved by the method of moments 

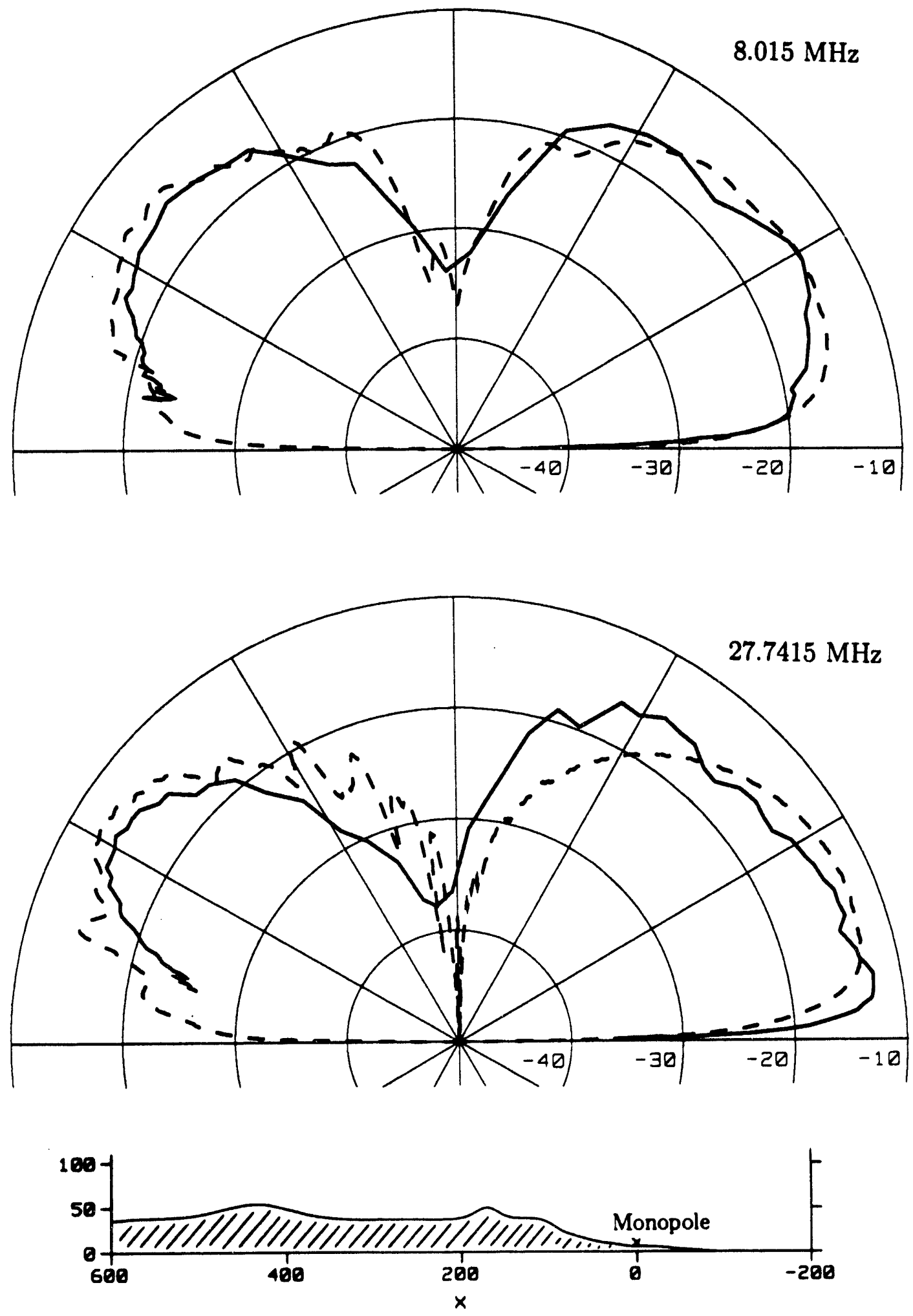

Fig. 26. Radiation pattern of a vertical monopole at the West Valley site at Cedar Valley, comparing the measured pattern (solid) with the 2D PO result (dashed). The patterns are in $\mathrm{dB}$ and were normalized to match their maximum values. 

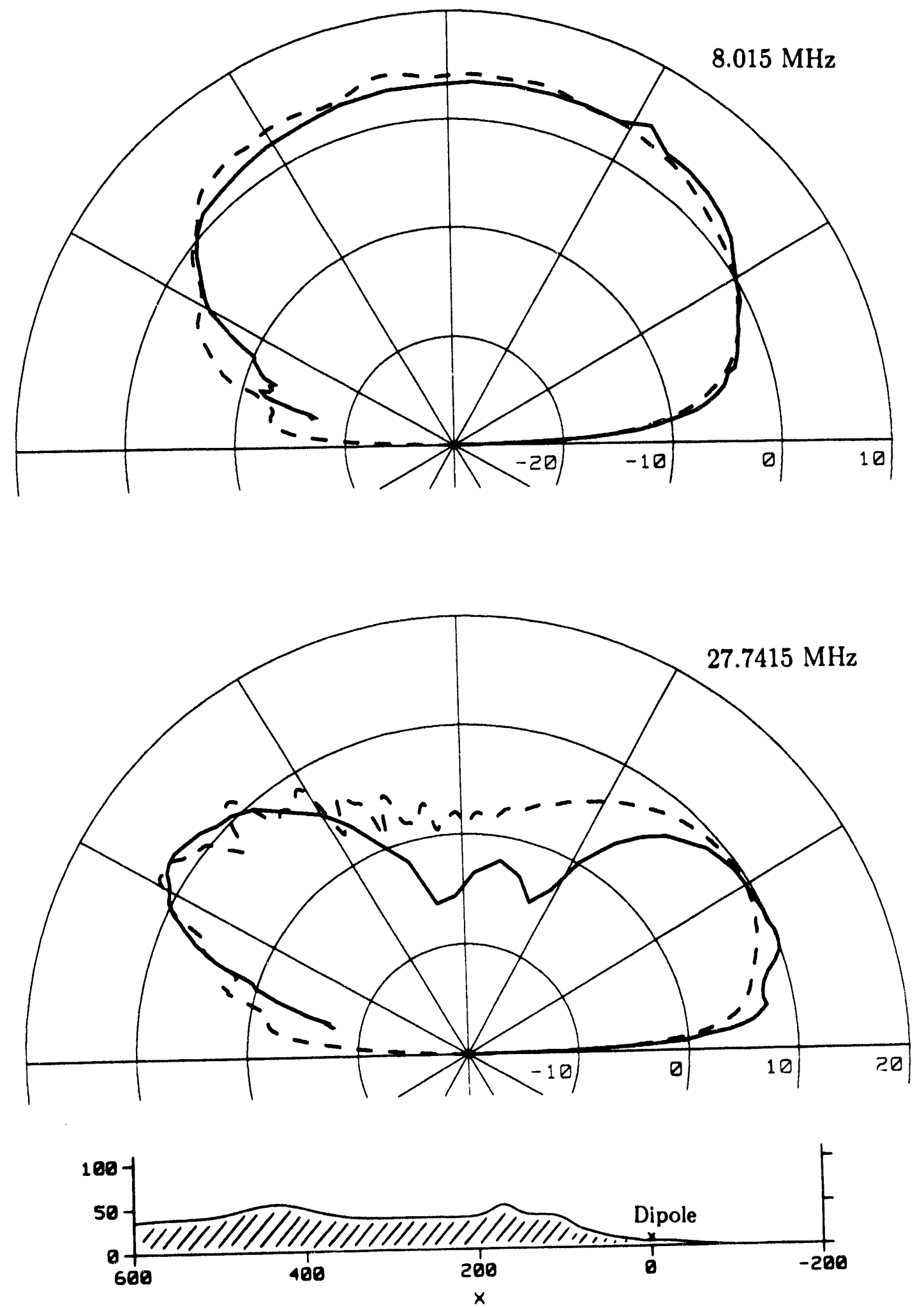

Fig. 27. Radiation pattern of a horizontal dipole at the West Valley site at Cedar Valley, comparing the measured pattern (solid) with the 2D PO result (dashed). The patterns are in $\mathrm{dB}$ and were normalized to match their maximum values. 

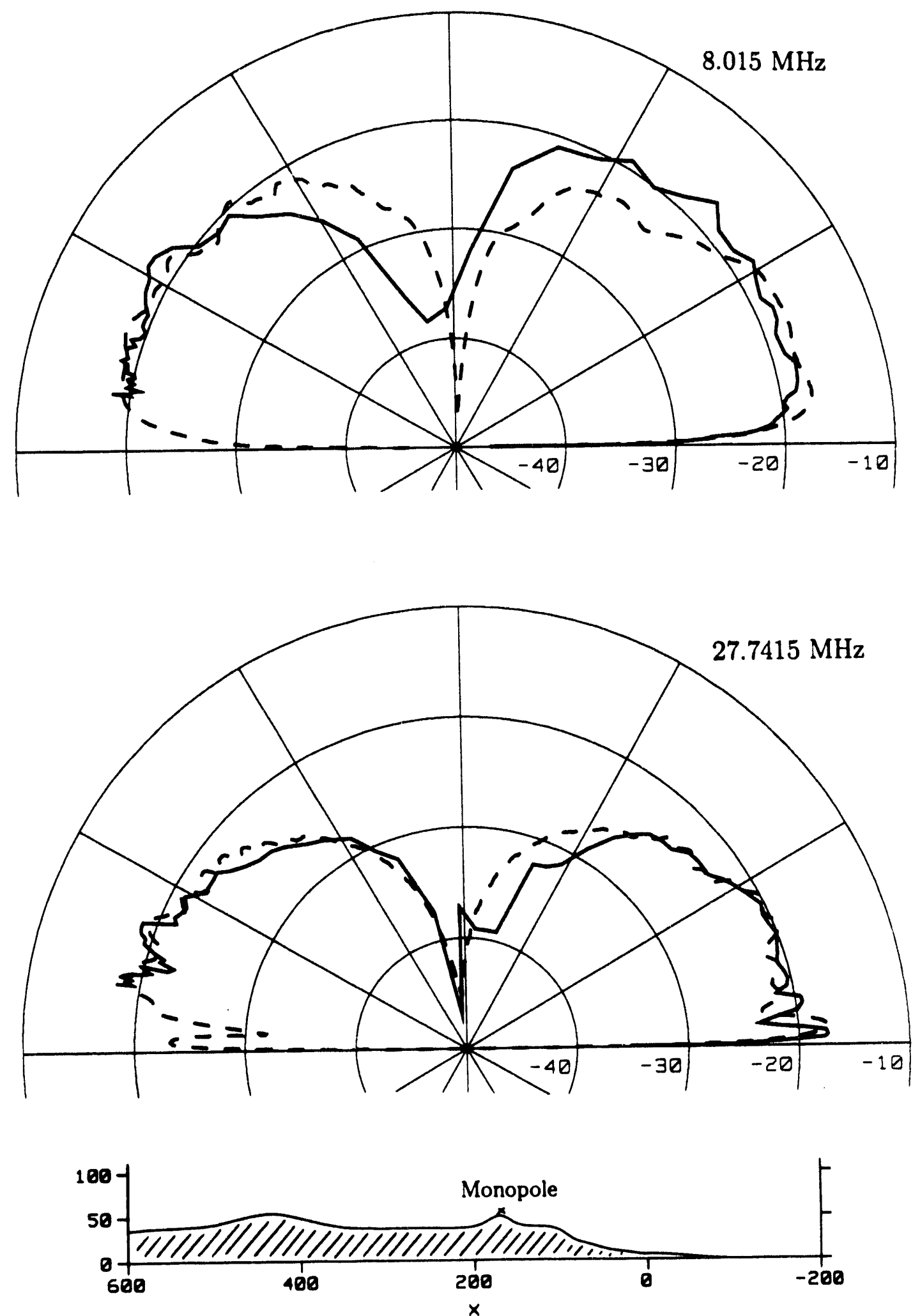

Fig. 28. Radiation pattern of a vertical monopole at the Hill Top site at Cedar Valley, comparing the measured pattern (solid) with the 2D PO result (dashed). The patterns are in $\mathrm{dB}$ and were normalized to match their maximum values. 

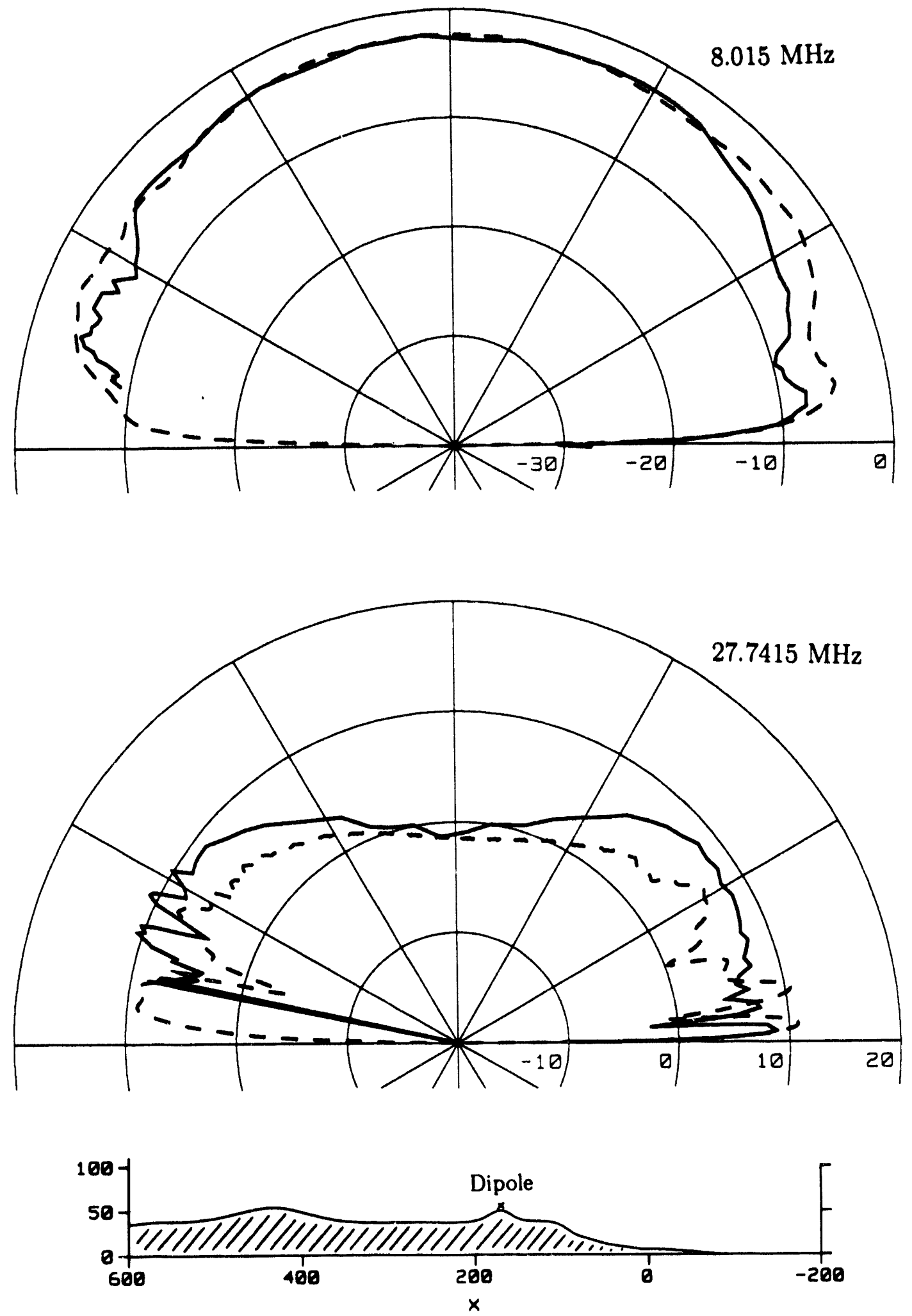

Fig. 29. Radiation pattern of a horizontal dipole at the Hill Top site at Cedar Valley, comparing the measured pattern (solid) with the 2D PO result (dashed). The patterns are in $\mathrm{dB}$ and were normalized to match their maximum values. 
with pulse expansion functions and point matching, and with the first and last pulse basis function extended to minus and plus infinity to reduce edge reflections. The integral equation solutions were found to be in good agreement with the exact result for a fat impedance plane and with results of the code WAGNER for surfaces with hills. The radiation patterns from the integral equation and PO codes were compared for vertical and horizontal antennas at the base of a simple Gaussian hill and also on top of the hill, and at similar positions on an actual terrain profile from the Cedar Valley site. Generally good agreement was found between integral equation and PO solutions in all cases. Comparisons of the PO results with measurements taken at Cedar Valley showed agreement in the major features of the patterns.

The PO codes are set up to model terrain of arbitrary shape and to read antenna geometry and currents from a NEC-MoM solution to represent the source. Alternatively, point or line sources can be used. The terrain shape is described by generating a file containing elevations at a number of points read from a topographic map, and the code applies cubicspline interpolation to the points entered to determine the surface for the PO solution. The formats of the input files and the use of the codes are described in appendices to this report.

\section{Acknowledgment}

The work reported here and the preparation of this report were funded by the Naval Security Group Command through CDR Gus K. Lott.

\section{References}

[1] G. J. Burke and A. J. Poggio, "Numerical Electromagnetics Code (NEC) - Method of Moments," Tech. Rept. UCID-18834, Lawrence Livermore Nat. Lab., Jan. 1981.

[2] G. J. Burke, "Numerical Modeling of HF Skywave Radiation from Antennas in Irregular Terrain," Tech. Rept. UCRL-ID-105656, Lawrence Livermore Nat. Lab., Nov. 1990.

[3] S. Marcus, "A finite difference method for propagation predictions over irregular terrain," Joint IEEE AP-S International Symposium and URSI Meeting, London, Ontario, Canada, July 1991.

[4] R. Janaswamy, "Application of the measured Equation of Invariance to Wave Propagation over Irregular, Inhomogeneous Terrain," Naval Postgraduate School Report NPSEC-93-018, October 1993.

[5] R. Mittra and A. Rushdi, "An Efficient Approach for Computing the Geometrical Optics Field Reflected from a Numerically Specified Surface," IEEE Trans. Antennas and Propagation, Vol. AP-27, No. 6, pp. 871-877, Nov. 1979.

[6] R. J. Marhefka and W. D. Burnside, "Numerical Electromagnetics Code - Basic Scattering Code (Version 2), Part I: User's Manual," The Ohio State University, ElectroScience 
Lab. Tech. Report 712242-14, Dec. 1982.

(7) J. K. Breakall, R. J. Lunnen, D. H. Werner, J. S. Young and T. A. Erdley, "Interim Report, High Frequency (HF) Antenna Siting Effects in Irregular Terrain," Penn State University Applied Research Laboratory, 15 Dec. 1991.

[8] G. A. Hufford, "An Integral Equation Approach to the Problem of Wave Propagation over an Irregular Terrain," Quart. Appl. Math., Vol. 9, No. 4, pp. 391-404, 1952.

[9] R. H. Ott and L. A. Berry, "An Alternative Integral Equation for Propagation over Irregular Terrain," Radio Science, Vol. 5, No. 5, pp. 767-771, May 1970.

[10] R. H. Ott, "An Alternative Integral Equation for Propagation over Irregular Terrain, 2," Radio Science, Vol. 6, No. 4, pp. 429-435, April 1971.

(11] R. H. Ott and G. J. Burke, "Comparison of Numerical Integration of RING Surface Fields and 1st Order Physical Optics for the Skywave Field of HF Antennas in the Vicinity of the Utah Test Site and an Electrically Large Gaussian Ridge," Conference Proceedings, 8th Annual Review of Progress in Applied Computational Electromagnetics, Monterey, CA, March 16-20, 1992.

[12] J. A. Stratton, Electromagnetic Theory, New York: McGraw-Hill, 1941.

[13] J. L. Volakis, "A Uniform Geometrical Theory of Diffraction for an Imperfectly Conducting Half-Plane," IEEE Trans. Antennas and Propagation, Vol. AP-34, pp. 172-180, 1986.

[14] A. J. Poggio and E. K. Miller, "Integral Equation Solutions of Three-Dimensional Scattering Problems," Chapt. IV in Computer Techniques for Electromagnetics, edited by R. Mittra, Pergamon Press, NY, 1973.

[15] E. K. Miller, "A Variable Interval Width Quadrature Technique Based on Romberg's Method," Journal of Comp. Phys., Vol. 5, No. 2, April 1970.

[16] R. Janaswamy, "A Fredholm Integral Equation Method for Propagation Predictions Over Small Terrain Irregularities," IEEE Trans. Antennas and Propagation, Vol. AP. 40, pp. 1416-1422, Nov. 1992.

[17] R. H. Ott, "RING: An integral equation algorithm for HF-VHF radio wave propagation over irregular, inhomogeneous terrain," Radio Science, Vol. 27, No. 6, pp. 867-882, Nov.Dec. 1992.

[18] J. R. Wait, Electromagnetic Waves in Stratified Media, Oxford: Pergamon, 1962.

[19] L. A. Berry, "Radio Propagation over a Gaussian-Shaped Ridge," IEEE Trans. Antennas and Propagation, Vol. AP-15, pp. 701-703, Sept. 1967. 
[20] R. H. Ott, "A New Method for Predicting HF Ground Wave Attenuation Over Inhomogeneous, Irregular Terrain," U. S. Dept. of Commerce, Office of Telecommunications, Report OT/ITSRR 7, January 1971.

[21] R. M. Bevensee, "Compensation-Theorem Analyses of Ground Wave Propagation over Irregular, Inhomogeneous Terrains," Journal of Atmospheric and Terrestrial Physics, Vol. 51, No. 9/10, pp. 797-810, 1989.

[22] G. H. Hagn, D. L. Faust, "Cedar Valley Antenna Tests, Covering Period 10-24 May 1990," SRI International, Arlington VA, 28 May 1990. 


\section{APPENDIX A}

\section{Diffraction Coefficients for a Lossy Half-Plane}

The UTD diffraction coefficients developed by Volakis [A $\mid$ ) for a finitely conducting half-plane with impedance boundary condition are summarized in this Appendix. The formulas are considerably more complicated than for a perfectly conducting half-plane, but remain correct in the limit of perfect conductivity. Volakis developed the UTD solution for incidence at an arbitrary angle to the edge and also gave a simplified form for normal incidence. The normal incidence form which was used in the 2D PO code will be given first.

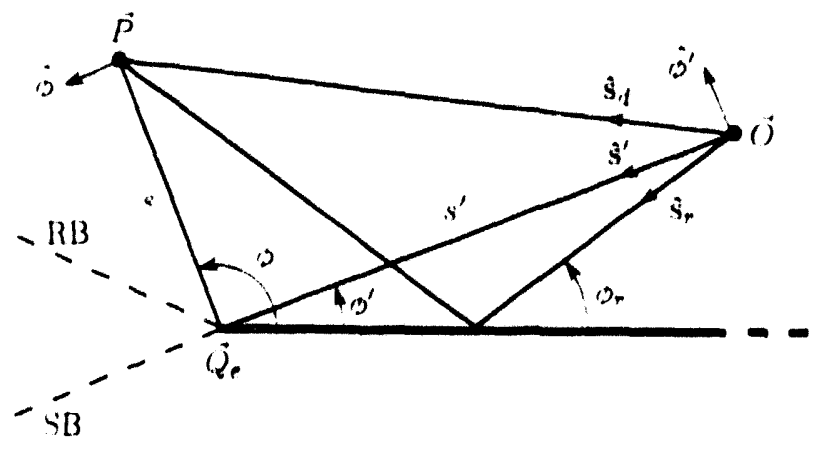

Fig. A1 Geometry for diffraction by a half-plane with incidence normal to the edge.

\section{Incidence normal to the edge.}

The geometry for the ray incident normal to the edge is shown in Figure A1. The free space wavenumber is $k=\omega / c$ and $\eta=Z_{s} / Z_{0}$ is the normalized surface impedance of the half-plane. For the terrain model $\eta=\sqrt{1 / \tilde{\epsilon}_{g}}$ where $\tilde{\epsilon}_{g}=\epsilon_{r}-j \sigma / \omega \epsilon_{0}$ with $\epsilon_{r}$ and $\sigma$ the relative permittivity and conductivity of the ground. If the incident field at the edge is $\mathbf{E}^{i}\left(\mathbf{Q}_{e}\right)$, the diffracted field at $\mathbf{P}$ is

$$
\begin{aligned}
\mathbf{E}^{d}(\mathbf{P}) \sim-\mathbf{E}^{i}\left(\mathbf{Q}_{e}\right) \cdot & {\left[\hat{\beta}_{0}^{\prime} \hat{\beta}_{0} D\left(\phi, \phi^{\prime} ; \pi / 2, \eta\right)+\hat{\phi}^{\prime} \hat{\phi} D\left(\phi, \phi^{\prime} ; \pi / 2,1 / \eta\right)\right] A_{1}(s) } \\
D\left(\phi, \phi^{\prime} ; \pi / 2, \eta\right)= & \frac{e^{-j \pi / 4}}{2 \sqrt{2 \pi k}}\left[C_{1}\left(\phi, \phi^{\prime} ; \eta\right) \sec \left(\beta^{-} / 2\right) F\left(2 k L \cos ^{2} \beta^{-} / 2\right)\right. \\
& \left.+C_{2}\left(\phi, \phi^{\prime} ; \eta\right) \sec \left(\beta^{+} / 2\right) F\left(2 k L \cos ^{2} \beta^{+} / 2\right)\right]
\end{aligned}
$$

where $\beta^{ \pm}=\phi \pm \phi^{\prime}$ and

$$
\begin{aligned}
& C_{1}\left(\phi, \phi^{\prime} ; \eta\right)=\frac{2 \eta \sin \phi / 2 \sin \phi^{\prime} / 2+1}{2 \sin \phi / 2 \sin \phi^{\prime} / 2} K_{+}(\phi ; \pi / 2, \eta) K_{+}\left(\phi^{\prime} ; \pi / 2, \eta\right) \\
& C_{2}\left(\phi, \phi^{\prime} ; \eta\right)=\frac{2 \eta \sin \phi / 2 \sin \phi^{\prime} / 2-1}{2 \sin \phi / 2 \sin \phi^{\prime} / 2} K_{+}(\phi ; \pi / 2, \eta) K_{+}\left(\phi^{\prime} ; \pi / 2, \eta\right) .
\end{aligned}
$$

$F$ is the Fresnel integral

$$
F(x)=2 j|\sqrt{x}| e^{j x} \int_{|\sqrt{x}|}^{\infty} e^{-j \tau^{2}} d \tau
$$

and $L$ is the distance parameter

$$
L= \begin{cases}s \sin ^{2} \beta_{0} & \text { for a plane wave incident } \\ \frac{s^{\prime} s}{s+s} & \text { for cylindrical waves } \\ \frac{s^{\prime} s \sin ^{2} \beta_{0}}{s+s} & \text { for conical or spherical waves }\end{cases}
$$


while $A_{1}$ contains the spreading and phase factors

$$
A_{1}(s)= \begin{cases}e^{-j k s} / \sqrt{s} & \text { for plane, cylindrical or conical waves incident } \\ e^{-j k s}\left|\frac{s^{\prime}}{s\left(s^{\prime}+s\right)}\right|^{1 / 2} & \text { for a spherical wave incident. }\end{cases}
$$

$K_{+}$is the "split function" for the Wiener-Hopf solution and is given by

$$
K_{+}\left(\phi ; \beta_{0}, \eta\right)=\frac{2[2 \cos \chi(1-\cos \phi)]^{1 / 2}}{\left(\sqrt{2} \sin \frac{\phi-\chi}{2}+1\right)\left(\sqrt{2} \sin \frac{\phi+\chi}{2}+1\right)}\left[\frac{\psi_{\pi}(\pi-\phi+\chi) \psi_{\pi}(\pi-\phi-\chi)}{\psi_{\pi}(\pi / 2) \psi_{\pi}(\pi / 2)}\right]^{2}
$$

where

$$
\cos \chi=1 /\left(\eta \sin \beta_{0}\right)
$$

and

$$
\begin{aligned}
\psi_{\pi}(\alpha) & =\exp \left[\frac{-1}{8 \pi} \int_{0}^{\alpha} \frac{\pi \sin u-2 \sqrt{2} \pi \sin u / 2+2 u}{\cos u} d u\right] \\
& \approx\left\{\begin{array}{c}
1-0.0139004 \alpha^{2}-1.15772\left(10^{-5}\right) \alpha^{4}, \quad 0 \leq \sigma \leq \pi / 2, \tau \leq 4.6 \\
1.05302\left[\cos \frac{1}{4}\left(\alpha-j \gamma_{0}\right)\right]^{1 / 2} \exp \left(\frac{j \alpha}{2 \pi} e^{j \alpha}\right) \\
0 \leq \sigma \leq \pi / 2, \quad \tau>4.6
\end{array}\right.
\end{aligned}
$$

where $\alpha=\sigma+j \tau$ and $\gamma_{0}=0.69315$. For other arguments the function $\psi_{\pi}$ can be evaluated from (A4) and the identity

$$
\psi_{\pi}(\alpha)=0.93242 \frac{\cos \left(\frac{\alpha}{4}-\frac{\pi}{8}\right)}{\psi_{\pi}(\alpha-\pi)}
$$

with the relations $\psi_{\pi}(\alpha)=\psi_{\pi}(-\alpha)$ and $\psi_{\pi}\left(\alpha^{*}\right)=\psi_{\pi}^{*}(\alpha)$, where $\alpha^{*}$ indicates the conjugate.

Skew incidence

The geometry for diffraction at skew incidence is shown in Figure A2. If the halfplane is perfectly conducting and the incident field at the diffraction point $\left(\beta_{0}^{\prime}=\beta_{0}\right)$ is $\mathbf{E}^{i}\left(\mathbf{Q}_{e}\right)=E_{\beta_{0}^{\prime}}^{i}\left(\mathbf{Q}_{e}\right) \hat{\beta}_{0}^{\prime}+E_{\phi^{\prime}}^{i}\left(\mathbf{Q}_{e}\right) \hat{\phi}_{0}$ the diffracted field at $\mathbf{P}$ is $[\mathrm{A} 2]$

$$
\left[\begin{array}{c}
E_{\beta_{0}}^{d}(\mathbf{P}) \\
E_{\phi}^{d}(\mathbf{P})
\end{array}\right] \sim-A_{1}(s)\left[\begin{array}{cc}
D_{s}\left(\phi, \phi^{\prime} ; \beta_{0}\right) & 0 \\
0 & D_{h}\left(\phi, \phi^{\prime} ; \beta_{0}\right)
\end{array}\right] \cdot\left[\begin{array}{c}
E_{\beta_{\beta^{\prime}}^{\prime}}^{i}\left(\mathbf{Q}_{E}\right) \\
E_{\phi^{i}}^{i}\left(\mathbf{Q}_{E}\right)
\end{array}\right]
$$

$D_{s}$ and $D_{h}$ are the diffraction coefficients for soft and hard boundary conditions

$$
\begin{aligned}
D_{h}\left(\phi, \phi^{\prime} ; \beta_{0}\right)=\frac{-e^{-j \pi / 4}}{2 \sqrt{2 \pi k} \sin \beta_{0}} & {\left[\sec \left(\frac{\phi-\phi^{\prime}}{2}\right) F\left[k L a\left(\phi-\phi^{\prime}\right)\right]\right.} \\
& \left.\mp \sec \left(\frac{\phi+\phi^{\prime}}{2}\right) F\left[k L a\left(\phi+\phi^{\prime}\right)\right]\right]
\end{aligned}
$$




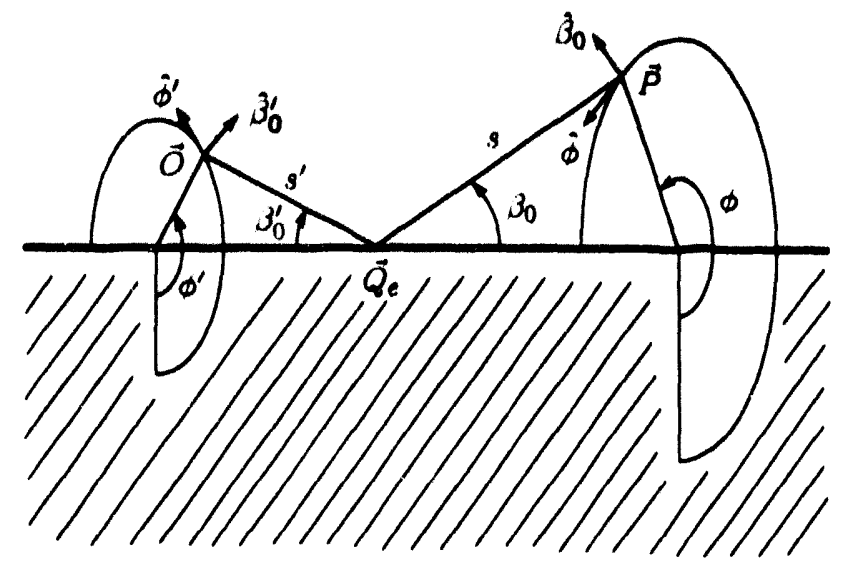

Fig. 1. Geometry for diffraction by a half-plane with skew incidence.

where $a\left(\phi \pm \phi^{\prime}\right)=2 \cos ^{2}\left[\left(\phi \pm \phi^{\prime}\right) / 2\right]$. When the half-plane has finite conductivity the $\hat{\phi}$ and $\hat{\beta}_{0}$ components of field are coupled so that the diffracted field is

$$
\left[\begin{array}{c}
E_{\beta_{0}}^{\alpha}(\mathbf{P}) \\
E_{\phi}^{d}(\mathbf{P})
\end{array}\right] \sim-A_{1}(s)\left[\begin{array}{cc}
D\left(\phi, \phi^{\prime} ; \beta_{0}, \eta\right) & D_{c}\left(\phi, \phi^{\prime} ; \beta_{0}, 1 / \eta\right) \\
-D_{c}\left(\phi, \phi^{\prime} ; \beta_{0}, \eta\right) & D\left(\phi, \phi^{\prime} ; \beta_{0}, 1 / \eta\right)
\end{array}\right] \cdot\left[\begin{array}{c}
E_{\beta_{0}^{\prime}}^{i}\left(\mathbf{Q}_{E}\right) \\
E_{\phi^{i}}^{i}\left(\mathbf{Q}_{E}\right)
\end{array}\right]
$$

The diffraction coefficients from [A1] are

$$
\begin{aligned}
& D\left(\phi, \phi^{\prime} ; \beta_{0}, \eta\right)=A_{2}\left(\phi, \phi^{\prime} ; \beta_{0}\right)\left[E\left(\phi, \phi^{\prime} ; \beta_{0}, \eta\right)+\cos \beta_{0} F\left(\phi, \phi^{\prime} ; \beta_{0}, 1 / \eta\right)\right] \\
& D_{c}\left(\phi, \phi^{\prime} ; \beta_{0}, \eta\right)=A_{2}\left(\phi, \phi^{\prime} ; \beta_{0}\right)\left[G\left(\phi, \phi^{\prime} ; \beta_{0}, \eta\right)+\cos \beta_{0} H\left(\phi, \phi^{\prime} ; \beta_{0}, 1 / \eta\right)\right]
\end{aligned}
$$

where

$$
A_{2}\left(\phi, \phi^{\prime} ; \beta_{0}\right)=\frac{e^{-j \pi / 4}}{\sqrt{2 \pi k}\left(1-\sin ^{2} \beta_{0} \sin ^{2} \phi^{\prime}\right)\left(1-\sin ^{2} \beta_{0} \sin ^{2} \phi\right)}
$$

and

$$
\begin{gathered}
{\left[\begin{array}{l}
E^{\eta} \\
G^{\eta}
\end{array}\right]=-\left[\begin{array}{cc}
\cos \phi \cos \phi^{\prime} & -\cos \beta_{0} \sin \phi \cos \phi^{\prime} \\
\cos \beta_{0} \sin \phi \cos \phi^{\prime} & \cos \phi \cos \phi^{\prime}
\end{array}\right] \cdot\left[\begin{array}{l}
U^{\eta} \\
V_{\eta}
\end{array}\right]} \\
{\left[\begin{array}{l}
F_{\eta} \\
H_{\eta}
\end{array}\right]=-\left[\begin{array}{cc}
\cos \beta_{0} \sin \phi \sin \phi^{\prime} & \cos \phi \sin \phi^{\prime} \\
-\cos \phi \sin \phi^{\prime} & \cos \beta_{0} \sin \phi \sin \phi^{\prime}
\end{array}\right] \cdot\left[\begin{array}{l}
U_{\eta} \\
V^{\eta}
\end{array}\right]}
\end{gathered}
$$

The notation $E^{\eta}=E\left(\phi, \phi^{\prime} ; \beta_{0}, \eta\right), E_{\eta}=E\left(\phi, \phi^{\prime} ; \beta_{0}, 1 / \eta\right)$, etc. has been used in the above equations.

The UTD forms that Volakis developed for these coefficients follow. $U$ is evaluated as

$$
U^{\eta}=U\left(\phi, \phi^{\prime} ; \beta_{0}, \eta\right)=U_{1}^{\eta}+U_{2}^{\eta}
$$

where

$$
U_{1}^{\eta}=\cos \phi \cos \phi^{\prime} D^{\mathrm{I}}(\eta)+C_{13}\left(\phi, \phi^{\prime} ; \beta_{0}, \eta\right) C_{s}\left(\phi, \phi^{\prime} ; \beta_{0}\right)
$$


with

$$
\begin{aligned}
D^{\mathrm{I}}(\eta)= & 2 C_{11}\left(\phi, \phi^{\prime} ; \beta_{0}, \eta\right) \sec \left(\beta^{-} / 2\right) F\left(2 k L \cos ^{2} \beta^{-} / 2\right) \\
+ & 2 C_{12}\left(\phi, \phi^{\prime} ; \beta_{0}, \eta\right) \sec \left(\beta^{+} / 2\right) F\left(2 k L \cos ^{2} \beta^{+} / 2\right)
\end{aligned}
$$

and

$$
\begin{gathered}
C_{11}\left(\phi, \phi^{\prime} ; \beta_{0}, \eta\right)=\frac{2 \eta \sin \beta_{0} \sin \phi / 2 \sin \phi^{\prime} / 2+1}{8 \sin \beta_{0} \sin \phi / 2 \sin \phi^{\prime} / 2} K_{+}\left(\phi ; \beta_{0}, \eta\right) K_{+}\left(\phi^{\prime} ; \beta_{0}, \eta\right) \\
C_{12}\left(\phi, \phi^{\prime} ; \beta_{0}, \eta\right)=\frac{2 \eta \sin \beta_{0} \sin \phi / 2 \sin \phi^{\prime} / 2-1}{8 \sin \beta_{0} \sin \phi / 2 \sin \phi^{\prime} / 2} K_{+}\left(\phi ; \beta_{0}, \eta\right) K_{+}\left(\phi^{\prime} ; \beta_{0}, \eta\right) \\
C_{13}\left(\phi, \phi^{\prime} ; \beta_{0}, \eta\right)=\frac{\sin \beta_{0} \cos \beta_{0}\left[2 \eta \sin \beta_{0} \cos \phi / 2 \cos \phi^{\prime} / 2\left(\cos \beta_{0}-\sin 2 \gamma^{\eta}\right)\left(\cos \phi+\cos \phi^{\prime}\right)\right.}{2 \sin \phi / 2 \sin \phi^{\prime} / 2\left(\sin \beta_{0}+\cos 2 \gamma^{\eta}\right)} .
\end{gathered}
$$

The function $\gamma^{\eta}=\gamma\left(\beta_{0}, \eta\right)$ is evaluated as

$$
\gamma^{\eta}=-\gamma_{\eta}=-j \log _{e}\left\{K_{+}\left(\theta ; \beta_{0}, 1 / \eta\right) /\left[\sqrt{\eta} K_{+}\left(\theta ; \beta_{0}, \eta\right)\right]\right\}
$$

with

$$
\theta=\pi / 2+j \log _{e}\left(\tan \beta_{0} / 2\right)
$$

$U_{2}$ is evaluated as

$$
U_{2}^{\eta}=\cos ^{2} \beta_{0}\left(1+\cos \phi \cos \phi^{\prime}\right) D^{\mathrm{II}}(\eta)+C_{23}\left(\phi, \phi^{\prime} ; \beta_{0}, \eta\right) C_{h}\left(\phi, \phi^{\prime} ; \beta_{0}\right)
$$

where

$$
\begin{aligned}
D^{\mathrm{II}}(\eta)= & 2 C_{21}\left(\phi, \phi^{\prime} ; \beta_{0}, \eta\right) \sec \left(\beta^{-} / 2\right) F\left(2 k L \cos ^{2} \beta^{-} / 2\right) \\
& +2 C_{22}\left(\phi, \phi^{\prime} ; \beta_{0}, \eta\right) \sec \left(\beta^{+} / 2\right) F\left(2 k L \cos ^{2} \beta^{+} / 2\right)
\end{aligned}
$$

and

$$
\begin{aligned}
C_{21}\left(\phi, \phi^{\prime} ; \beta_{0}, \eta\right)= & \frac{\sin \phi / 2 \sin \phi^{\prime} / 2+2 \eta \sin \beta_{0} \cos ^{2} \phi / 2 \cos ^{2} \phi^{\prime} / 2}{2 \sin \beta_{0} \sin \phi \sin \phi^{\prime}} \\
& \cdot K_{+}\left(\phi ; \beta_{0}, \eta\right) K_{+}\left(\phi^{\prime} ; \beta_{0}, \eta\right) \\
C_{22}\left(\phi, \phi^{\prime} ; \beta_{0}, \eta\right)= & \frac{\sin \phi / 2 \sin \phi^{\prime} / 2-2 \eta \sin \beta_{0} \cos ^{2} \phi / 2 \cos ^{2} \phi^{\prime} / 2}{2 \sin \beta_{0} \sin \phi \sin \phi^{\prime}} \\
& \cdot K_{+}\left(\phi ; \beta_{0}, \eta\right) K_{+}\left(\phi^{\prime} ; \beta_{0}, \eta\right) \\
C_{23}\left(\phi, \phi^{\prime} ; \beta_{0}, \eta\right)= & -\frac{\sin \beta_{0} \cos \beta_{0}\left(\cos \beta_{0}+\sin 2 \gamma^{\eta}\right)\left(\cos \phi+\cos \phi^{\prime}\right)}{2\left(\sin \beta_{0}+\cos 2 \gamma^{\eta}\right) \cos \phi / 2 \cos \phi^{\prime} / 2} \\
& \cdot K_{+}\left(\phi ; \beta_{0}, \eta\right) K_{+}\left(\phi^{\prime} ; \beta_{0}, \eta\right) .
\end{aligned}
$$

The functions $C_{s}$ and $C_{h}$ are related to the soft and hard diffraction coefficients for the perfectly conducting half-plane as

$$
C_{\dot{h}}\left(\phi, \phi^{\prime} ; \beta_{0}\right)=e^{j \pi / 4} \sqrt{2 \pi k} D_{\dot{h}}\left(\phi, \phi^{\prime} ; \beta_{0}\right) .
$$

The UTD form of the $V$ function is

$$
V^{\eta}=V\left(\phi, \phi ; \beta_{0}, \eta\right)=C_{3}\left(\phi, \phi ; \beta_{0}, \eta\right) C_{9}\left(\phi, \phi^{\prime} ; \beta_{0}\right)+C_{4}\left(\phi, \phi ; \beta_{0}, \eta\right) C_{h}\left(\phi, \phi^{\prime} ; \beta_{0}\right)
$$


where

$$
\begin{aligned}
C_{3}\left(\phi, \phi^{\prime} ; \beta_{0}, \eta\right)= & \sin \beta_{0} \cos \beta_{0} \sqrt{2 \sin \beta_{0}} K_{+}\left(\phi ; \beta_{0}, \eta\right) K_{+}\left(\phi^{\prime} ; \beta_{0}, 1 / \eta\right) \\
& {\left[\frac{\sqrt{\eta} \sin \phi \cos \phi^{\prime}}{2 \sin \phi^{\prime} / 2 \cos \left(\frac{\pi}{4}-\frac{\beta_{0}}{2}-\gamma^{\eta}\right)}-\frac{\cos \phi \sin \phi^{\prime}}{2 \sqrt{\eta} \sin \phi / 2 \cos \left(\frac{\pi}{4}-\frac{\beta_{0}}{2}+\gamma^{\eta}\right)}\right] }
\end{aligned}
$$

and

$$
\begin{aligned}
C_{4}\left(\phi, \phi^{\prime} ; \beta_{0}, \eta\right)= & \sin \beta_{0} \cos \beta_{0} \sqrt{2 \sin \beta_{0}} K_{+}\left(\phi ; \beta_{0}, \eta\right) K_{+}\left(\phi^{\prime} ; \beta_{0}, 1 / \eta\right) \\
& {\left[\frac{\cos \phi / 2 \cos \phi^{\prime}}{\sqrt{\eta} \cos \left(\frac{\pi}{4}-\frac{\beta_{0}}{2}+\gamma^{\eta}\right)}-\frac{\sqrt{\eta} \cos \phi \cos \phi^{\prime} / 2}{\cos \left(\frac{\pi}{4}-\frac{\beta_{0}}{2}-\gamma^{\eta}\right)}\right] . }
\end{aligned}
$$

References

[A1] J. L. Volakis, "A Uniform Geometrical Theory of Diffraction for an Imperfectly Conducting Half-Plane," IEEE Trans. Antennas and Propagation, Vol. AP -34, pp. 172-180, 1986.

[A2] J. B. Keller, "Geometrical Theory of Diffraction," J. Opt. Soc. Am., Vol. 52, pp. 116$130,1962$.

49 


\section{APPENDIX B}

\section{Input for the Physical Optics Codes TPO2D and TPO3D}

To compute the radiation pattern of an antenna located in irregular terrain the PO codes need a description of the terrain shape and electrical parameters of the ground, the antenna dimensions and currents and the transmitting frequency. In addition, the limits and number of increments for integration over the terrain surface must be set to meet requirements for solution accuracy. Both the 2D and 3D programs read six lines of input from the terminal, and prompts are displayed for the required parameters. Since the terrain description may involve a large amount of data it is read from a file, and only the file name is entered from the terminal. The formats of the terrain data files for 2D and 3D are described in Section B.3 of this appendix.

A name can also be entered for a file containing the dimensions and currents for an arbitrary antenna. This file is produced by running a modified version of the NEC momentmethod code. Alternatively a vertical or horizontal point source can be specified without the need for a file. When a file of NEC currents is read the antenna can be translated or rotated about the vertical axis to position it in the terrain coordinate system. Normally the antenna should be modeled over ground in NEC, and the height of the NEC origin above the terrain surface in the PO calculation (ZORG on input line 4) should be zero so that the antenna has the same height as when the currents were computed. However, for an antenna such as a half-wave dipole the height can probably be varied over a reasonable range without running NEC to recompute the antenna currents. In locating the antenna, the slope of the ground must be taken into account to ensure that no antenna segments extend below the ground surface. Also, the frequency used in the NEC run should be the same as that specified for the PO calculation, but if the frequencies differ a warning is printed and the PO solution will continue using the frequency on the first input line of the PO code.

The limits specified for the PO integration and the number of integration intervals will depend on the terrain shape and the wavelength at the transmitting frequency. The PO integration should cover all hills and valleys that may produce significant scattering. Beyond these limits the ground is considered flat, and reflections are computed from the UTD half-plane solution. The transmitting antenna can be located over the PO integration region or over the UTD half-planes. It is probably not a good idea to locate it very close to the boundary between the UTD and PO regions. The number of integration intervals must be chosen so that the interval width is sufficiently small relative to wavelength for accurate integration. The interval width in $x$ is (XMIN - XMAX)/(NXPT - 1) with the same relation for $y$ in code TPO3D. The width on the surface will be somewhat larger than this when the slope is non-zero, but it is usually sufficient to consider the width in $x$ for typical terrain slopes. From initial tests with the Cedar Valley Utah terrain at $27.7415 \mathrm{MHz}$, an interval width of $0.1 \lambda$, where $\lambda$ is the wavelength, appears to be conservative. Results obtained with an interval size of $0.2 \lambda$ were nearly indistinguishable from those with the smaller interval. With an interval of $0.4 \lambda$ the general pattern shape was produced, and errors were less that 
one $\mathrm{dB}$. However, since the ripple in the pattern due to scattering was also less than one $\mathrm{dB}$, the errors appeared significant.

The solution time will be approximately proportional to the number of integration intervals, NXPT in code TPO2D and NXPT $*$ NYPT in TPO3D. It is also proportional to the number of evaluations of the radiated field NTH.

\section{B.1. The 2D PO Code - TPO2D}

The code TPO2D reads six lines of input from the computer terminal. Prompts are displayed, and the data is read in free format using the FORTRAN READ $(*, *)$ form. Numbers can be separated by spaces or commas, but an entry must be made for every item even if the number is zero. The second and third input lines ask for the names of auxiliary files containing a description of the terrain profile and the source antenna currents. These files must be prepared in advance following the formats described in Section B.3 for the terrain data and in Section B.4 for the antenna dimensions and currents.

TPO2D writes the computed radiated fields to an output file named TPO2D.DAT. The program TPOPLT can then be run to plot the radiation patterns.

The input for running program TPO2D follows:

Prompt 1: FMHZ, EPSR, SIG >

FMHZ - frequency in MHz.

EPSR - relative permittivity of the earth $\epsilon_{r}$.

SIG - conductivity of the earth $\sigma$ in $\mathrm{S} / \mathrm{m}$. If a negative value is entered for SIG the complex permittivity of the earth is set to $\tilde{\epsilon}_{g}=\epsilon_{r}-j \sigma / \omega \epsilon_{o}=$ EPSR $-j|\mathrm{SIG}|$.

\section{Prompt 2: Enter name of terrain data file >}

Enter the name of the file containing the 2D terrain profile. The format of the file is described in Section B.3.

\section{Prompt 3: Enter name of NEC current file >}

To compute the fields due to an actual antenna modeled with the NEC MM code the name of the file containing the NEC current data should be entered. The format of the file is described in Section B.4. Alternatively, a simple point source can be used as the antenna. For a point source the following symbols can be entered in upper or lower case:

$\mathrm{X} \quad-$ for a horizontal point source in the $x$ direction

$\mathbf{Y}$ or $\mathbf{H}$ - for a horizontal point source in the $y$ direction

$\mathbf{Z}$ or $\mathbf{V}$ - for a vertical point source.

No file is read with the point-source options. The point sources, as well as a NEC antenna model, can be translated and rotated relative to the terrain coordinate system by the parameters on the next input line. 


\section{Prompt 4: XORG, ZORG, XANG >}

XORG - X position of the origin of the NEC coordinate system (or the point source) in the terrain coordinate system (meters).

ZORG - height of the origin of the NEC coordinate system (or the point source) above the ground surface at position XORG on the terrain (meters).

XANG - The $x$ axis of the antenna coordinate system from NEC is rotated by XANG degrees from the $x$ axis of the terrain. If a horizontal point source is used it will also be rotated by XANG degrees.

\section{Prompt 5: RAD, TH1, TH2, NTH, ISORC >}

RAD - distance in meters of the receiving antenna from the origin of the terrain coordinate system. The antenna sweeps an arc of radius RAD with angles defined by $\theta$.

TH1 - initial angle $\theta$ of the receiver in degrees. The angle $\theta=0^{\circ}$ is straight up, and $\theta=90^{\circ}$ is on the positive $x$ axis.

TH2 - final value of the angle $\theta$.

NTH - number of values of $\theta$ ranging from TH1 to TH2.

ISORC - flag selecting the orientation of the receiving antenna

1 for $\hat{\theta}$ polarization (in the vertical plane)

2 for horizontal cross-axis polarization (y directed)

3 for horizontal on-axis polarization ( $x$ directed)

4 for vertical polarization ( $\mathrm{z}$ directed)

\section{Prompt 6: XMIN, XMAX, NXPT >}

XMIN - minimum value of $x$ in meters for integration of the physical optics currents. A UTD half plane will be added from XMIN to $-\infty$.

XMAX - maximum value of $x$ in meters for integration of the physical optics currents. A UTD half plane will be added from XMAX to $+\infty$.

NXPT - number of intervals in the PO integration from XMIN to XMAX

\section{Array Dimension Limitations for code TPO2D}

The following array dimensions are set as PARAMETERS in the code TPO2D. If any are changed care should be taken to change all occurrences of the parameter to the same value throughout the code.

MAXANG - maximum number of $\theta$ values in the radiation pattern (NTH).

MAXSEG - maximum number of segments from a NEC data file.

MAXTR - maximum number of points that can be entered in the terrain description file.

NSPMAX - maximum number of increments in the PO integration (NXPT). 


\section{B.2. The 3D PO Code - TPO3D}

The input for TPO3D is very similar to that for the 2D code TPO2D. The radiated fields are written to an output file named TPO3D.DAT. The program TPOPLT can then be run to plot the radiation patterns by following the instructions in Section B.5.

The input for running program TPO3D follows:

\section{Prompt 1: FMHZ, EPSR, SIG >}

FMHZ - frequency in MHz.

EPSR - relative permittivity of the earth $\epsilon_{r}$.

SIG - conductivity of the earth $\sigma$ in $\mathrm{S} / \mathrm{m}$. If a negative value is entered for SIG the complex permittivity of the earth is set to $\tilde{\epsilon}_{g}=\epsilon_{r}-j \sigma / \omega \epsilon_{o}=$ EPSR $-j|\mathrm{SIG}|$.

\section{Prompt 2: Enter name of terrain data file >}

Enter the name of the file containing the 3D terrain profile. The format of the file is described in Section B.3.

\section{Prompt 3: Enter name of NEC current file >}

To compute the fields due to an actual antenna modeled with the NEC MM code the name of the file containing the NEC current data should be entered. The format of the file is described in Section B.4 and is the same as for the 2D code. Alternatively, a simple point source can be used as the antenna. For a point source the following symbols can be entered in upper or lower case:

$\mathbf{X} \quad-$ for a horizontal point source in the $x$ direction

$\mathbf{Y}$ or $\mathbf{H ~ - ~ f o r ~ a ~ h o r i z o n t a l ~ p o i n t ~ s o u r c e ~ i n ~ t h e ~} y$ direction

$\mathbf{Z}$ or $\mathbf{V}$ - for a vertical point source.

No file is read with the point-source options. The point sources, as well as a NEC antenna model, can be translated and rotated relative to the terrain coordinate system by the parameters on the next input line.

\section{Prompt 4: XORG, YORG, ZORG, XANG >}

XORG - X position of the origin of the NEC coordinate systcm (or the point source) in the terrain coordinate system (meters).

YORG - Y position of the origin of the NEC coordinate system (or the point source) in the terrain coordinate system (meters).

ZORG - height of the origin of the NEC coordinate system (or the point source) above the ground surface at position (XORG, YORG) on the terrain (meters).

XANG - The $x$ axis of the antenna coordinate system from NEC is rotated by XANG degrees from the $x$ axis of the terrain. If a horizontal point source is used it will also be rotated by XA.VG degrees. 
Prompt 5: RAD, TH1, TH2, NTH, PHI, ETA >

RAD - distance in meters of the receiving antenna from the origin of the terrain coordinate system. The antenna sweeps an arc of radius RAD defined by the spherical coordinate angles $\theta$ and $\phi$.

TH1 - initial angle $\theta$ of the receiver in degrees. The angle $\theta=0^{\circ}$ is straight up, and $\theta=90^{\circ}$ is on the horizon with the azimuth angle $\phi$.

TH2 - final value of the angle $\theta$.

NTH - number of values of $\theta$ ranging from TH1 to TH2.

PHI - azimuth angle $\phi$ in degrees measured from the $x$ axis.

ETA - polarization angle of the receiving dipole. For $\eta=0^{\circ}$ the dipole is in the $\hat{\theta}$ direction, and a positive ETA rotates the polarization in the right-hand direction in the transverse plane of the outward propagating wave.

Prompt 6: XMIN, XMAX, NXPT, YMIN, YMAX, NYPT, IPLOT >

XMIN - minimum value of $x$ in meters for integration of the physical optics currents. A UTD half plane will be added from $x=$ XMIN to $-\infty$ at $z=0$.

XMAX - maximum value of $x$ in meters for integration of the physical optics currents. A UTD half plane will be added from $x=\mathrm{XMAX}$ to $+\infty$ at $z=0$.

NXPT - number of intervals in the PO integration from XMIN to XMAX

YMIN - minimum value of $y$ in meters for integration of the physical optics currents. A UTD half plane will be added from $y=$ YMIN to $-\infty$ at $z=0$.

YMAX - maximum value of $y$ in meters for integration of the physical optics currents. A UTD half plane will be added from $y=$ YMAX to $+\infty$ at $z=0$.

NYPT - number of intervals in the PO integration from YMIN to YMAX

IPLOT - Flag to request a screen plot of the terrain surface: $=1$ to plot the surface, $=0$ otherwise. IPLOT must be zero if the program is run in batch mode.

\section{Array Dimension Limitations for code TPO3D}

The following array dimensions are set as PARAMETERS in the code TPO3D. If any are changed care should be taken to change all occurrences of the parameter to the same value throughout the code.

MAXANG - maximum number of $\theta$ values in the radiation pattern (NTH).

MAXGX - maximum number of increments in $x$ for the PO integration (.XXP).

MAXGY - maximum number of increments in $y$ for the PO integration (NYPT).

MAXSEG - maximum number of segments from a NEC data file.

NXMAX - maximum number of points that can be entered in any terrain profile parallel to the $x$ axis in the terrain description file (see Section B.3).

NYMAX - maximum number of profiles parallel to the $x$ axis that can be entered into the terrain description file.

\section{B.3. The Terrain Description Files}

To compute the scattering by irregular terrain the PO codes need a description of the 
terrain elevation as a function of position over the range of the $\mathrm{PO}$ integration. The electrical parameters of the ground could also be functions of position, but in the present codes they are restricted to single values. The codes obtain the elevation data from an auxiliary file containing elevations at selected points read from a map. Cubic-spline interpolation is applied to this data to obtain elevations at equally spaced points needed for the PO integration.

A topographic map was used to obtain the terrain data for the examples in this report, although more automated methods could be developed. For the 2D code TPO2D a line representing the $x$ axis is drawn on the map through the point under the transmitting antenna and in the direction of propagation. For the Cedar Valley site the line coincided with the helicopter flight path. A point on this line is chosen as $x=0$. This reference point may be chosen under the transmitting antenna but can be anywhere on the line. Points are then chosen along the line to define the important features of the terrain relief. These points will generally be on contour lines, peak elevations or other points where the elevation can be estimated. The positions of these points are then measured from the reference point $x=0$, and the $(x, z)$ pairs $(z=$ elevation) are entered into the file. The file format for $N$ points is illustrated below:

$$
\begin{array}{cc}
N & \\
X_{1} & Z_{1} \\
X_{2} & Z_{2} \\
\vdots & \vdots \\
X_{N} & Z_{N}
\end{array}
$$

The values are read using the FORTRAN $\operatorname{READ}(*, *)$ form. The $x$ and $z$ values must be in units of meters in code TPO2D. The values read from the topographic map for Cedar valley

\begin{tabular}{|c|c|c|}
\hline $\begin{array}{l}36, \\
-500 . .0 ., \\
-300 . .0 ., \\
-100 . .0 ., \\
-50 . .3 . \\
-10 . .6 ., \\
-5.66 ., \\
0.6 .6 \\
5.6 ., \\
20 . .8 . \\
47 . .12 ., \\
72 . .19 . . \\
88 ., 25 .,\end{array}$ & $\begin{array}{l}97 . .31 ., \\
112 . .37 ., \\
155 . .43 ., \\
170 . .50 . \\
188 . .43 . \\
220 . .37 . \\
245 . .37 . \\
295 . .37 . \\
320 . .37 . \\
378 . .43 . \\
433 . .54 . . \\
496 . .43 . .\end{array}$ & $\begin{array}{l}586 . .37 . . \\
627 . .31 . . \\
658 . .25 . . \\
700 . .20 . . \\
720 . .15 . . \\
740 . .10 . . \\
750 . .5 . . \\
760 . .1 . . \\
770 . .0 . . \\
780 . .0 . . \\
790.0 . . \\
1000 . .0 . .\end{array}$ \\
\hline
\end{tabular}
are shown in Table B.1.

Table B.1 Elevation values used for 2D calculations for the Cedar Valley site.

To develop a 3D terrain description for the code TPO3D a line is first drawn on the: map as the $x$ axis and a point on this line is chosen as the origin. Other lines are then drawn parallel to the $x$ axis at arbitrary intervals in $y$. Points are then chosen along the lines $t()$ define the important features of the terrain relief, and the $x$ and $y$ coordinates of each point and its elevation are entered into the terrain description file. When the file is read the $x$ 
and $y$ coordinates will be multiplied by the scale factor $X_{\text {scale }}$ and the elevations will be multiplied by $Z_{\text {scale. }}$. The coordinates may be entered in any units, but after scaling they must be in units of meters. Also, a reference value $Z_{0}$, in input units, will be subtracted from all elevations so that an input elevation of $Z_{0}$ will become 0 meters in the code. When the UTD frame, made up of four half-planes, is added from the limits of the PO integration to infinity the half-planes all have an elevation of zero. Hence to avoid a discontinuity in the surface the terrain described in the input file should have an elevation of $Z_{0}$ at the lower and upper limits of the PO integration in $x$ and $y$. If the defined surface does not reach the reference elevation at the integration limits the UTD frame will still provide a reflection from the $z=0$ plane, but the edges will introduce some extraneous ripples into the pattern.

The format of the input file for the 3D terrain model is illustrated bellow:

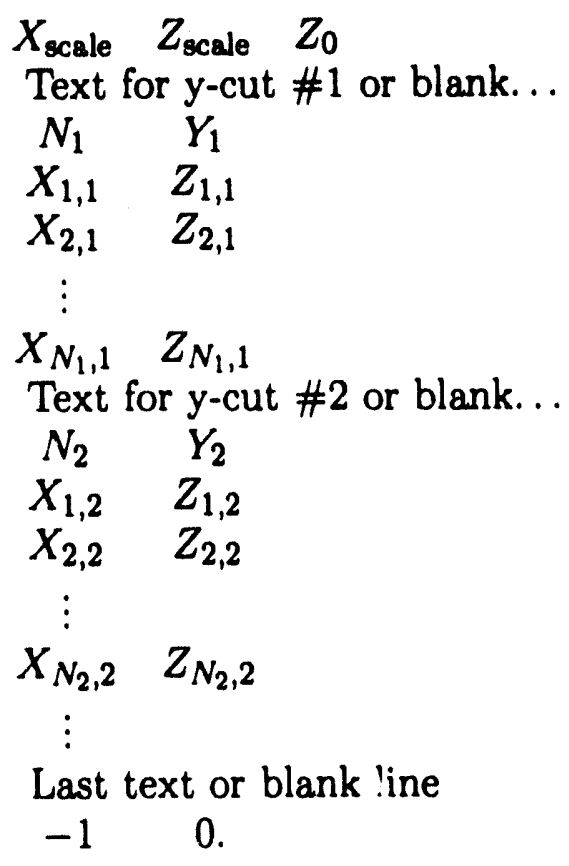

The first line contains the scale factors $X_{\text {scale }}$ for horizontal dimensions and $Z_{\text {scale }}$ for vertical dimensions, and the reference elevation $Z_{0}$. The remainder of the file contains tables of $(x, z)$ values along lines parallel to the $x$ axis. Each table begins with a line that can contain text describing the position of the cut. The next line, for table number $i$, contains the number of $(x, z)$ pairs, $N_{i}$ and the value of $y$ for the cut $Y_{i}$. Then $N_{i}$ lines with $x$ and $z$ values follow. After the last complete $y$-cut table, the file reading will terminate when a end-of-file is read of when a negative value for $N_{i}$ is read. The table read from the topographic map of Cedar Valley, and used to produce Figure 15, is show here as Table B.2.

\section{B.4. The Antenna Current File}

The antenna current file contains the description of the wire segments and segment currents making up a radiating antenna. Normally this file will be produced by running a version of the NEC antenna modeling code that has been modified to write the file. The subroutine GEOOTF is called after the call to DATAGN to write out the segment geometry 
Table B.2 Terrain data file for the 3D model of the Cedar Valley site.

\begin{tabular}{|c|c|c|c|c|}
\hline 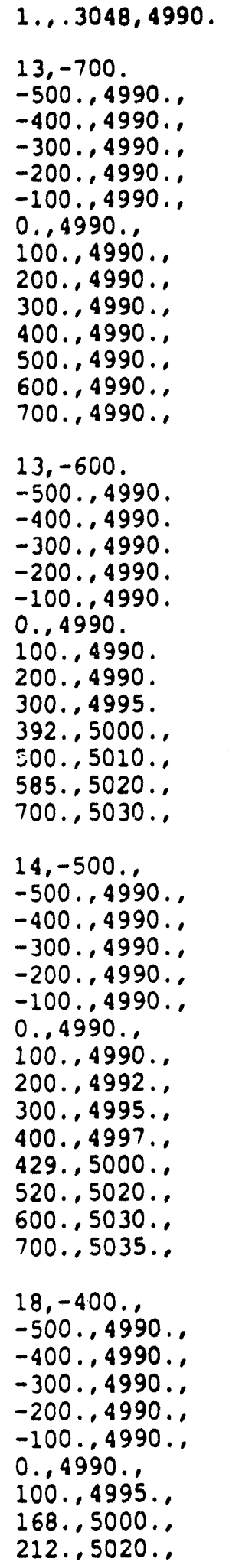 & 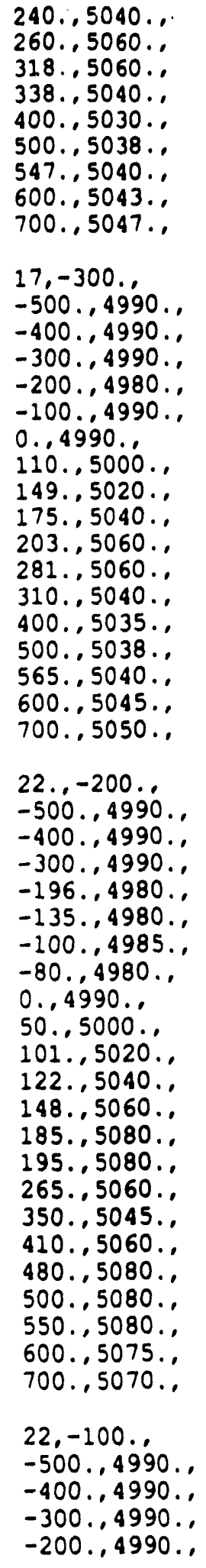 & 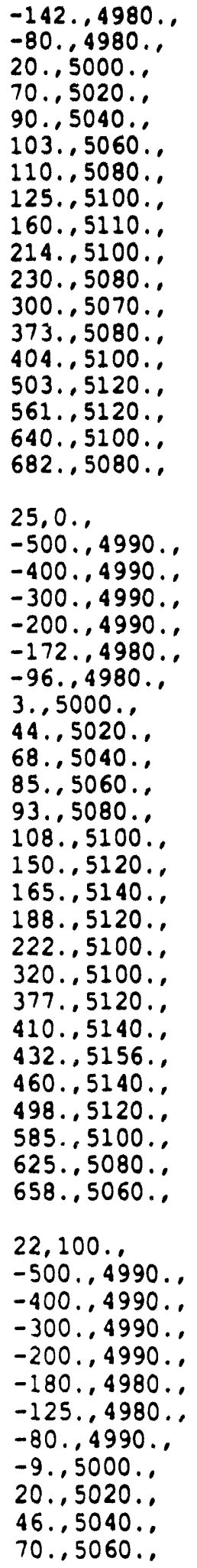 & 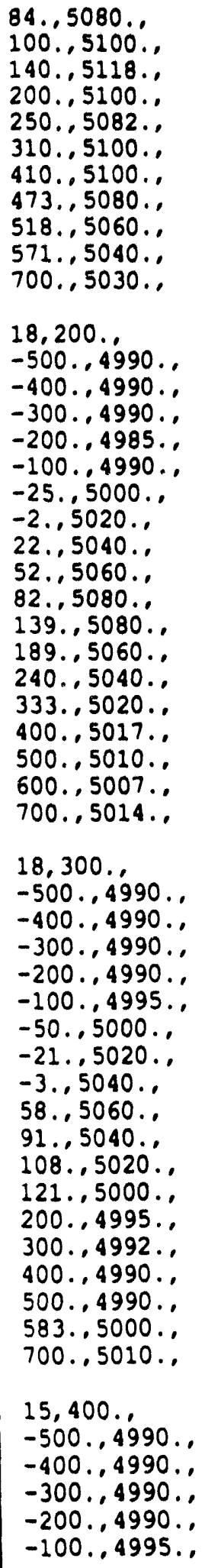 & 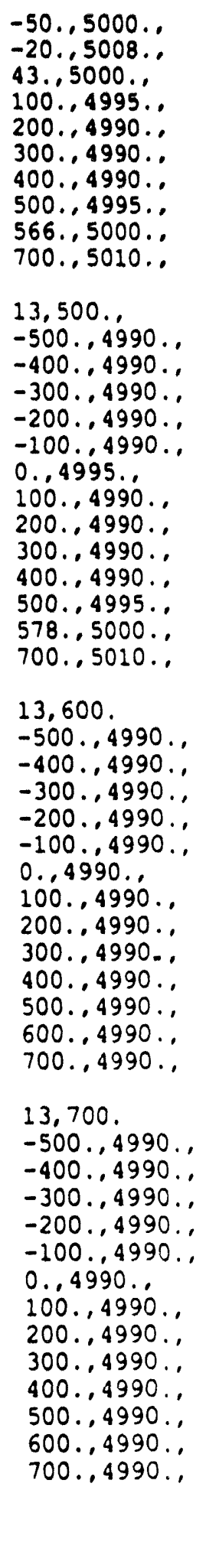 \\
\hline
\end{tabular}


data, and CUROTF is called after the call to NETWK to write the electrical parameters and currents. An example of the file for a monopole antenna with five segments follows:

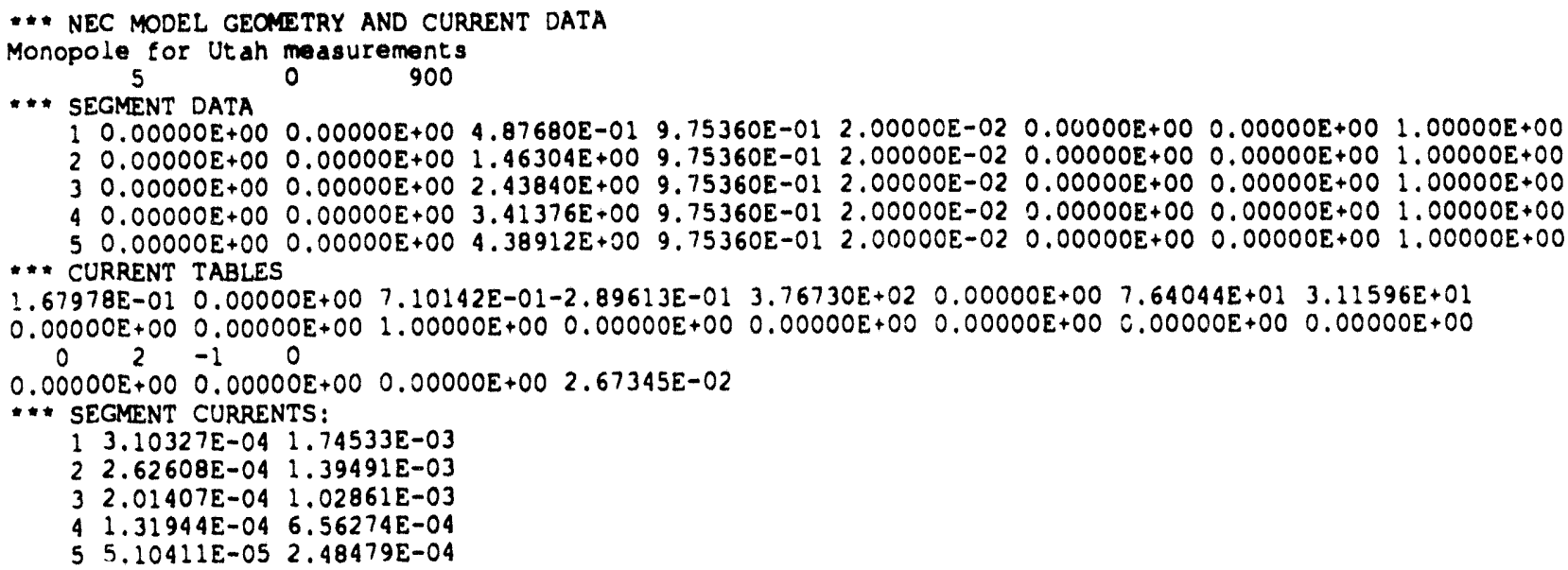

The lines starting with $* * *$ in this file are keys that the program reading the file must find. The second line is the title line from the NEC input file. The third line contains the number of segments, number of patches and the dimension of the data arrays (LD) when NEC was run. The lines following "*** SEGMENT DATA" are the segment number, $x, y$ and $z$ of the segment center, the segment length and radius, and the direction cosines of the segment.

Following "*** CURRENT TABLES" the parameters from the NEC common block /GND/ are printed in the following form:

$\begin{array}{llllll}\text { XKU } & \text { XKL } & \text { ETAU } & \text { ETAL } & & \\ \text { ZRAT2 } & \text { FRATI } & \text { CL } & \text { CH } & \text { SCRWL } & \text { SCRWR } \\ \text { NRADL } & \text { KSYMP } & \text { IFAR } & \text { IPERF } & & \\ \text { T1 } & \text { T2 } & \text { GSCAL } & & & \end{array}$

The first six of these parameters and $\mathrm{T} 1$ are complex numbers. Finally, following "*** SEGMENT CURRENTS" segment numbers and real and imaginary parts of the segment current are printed. If the model includes patches additional data not shown in the example will be printed. However, the PO codes do not at present use patch data. 

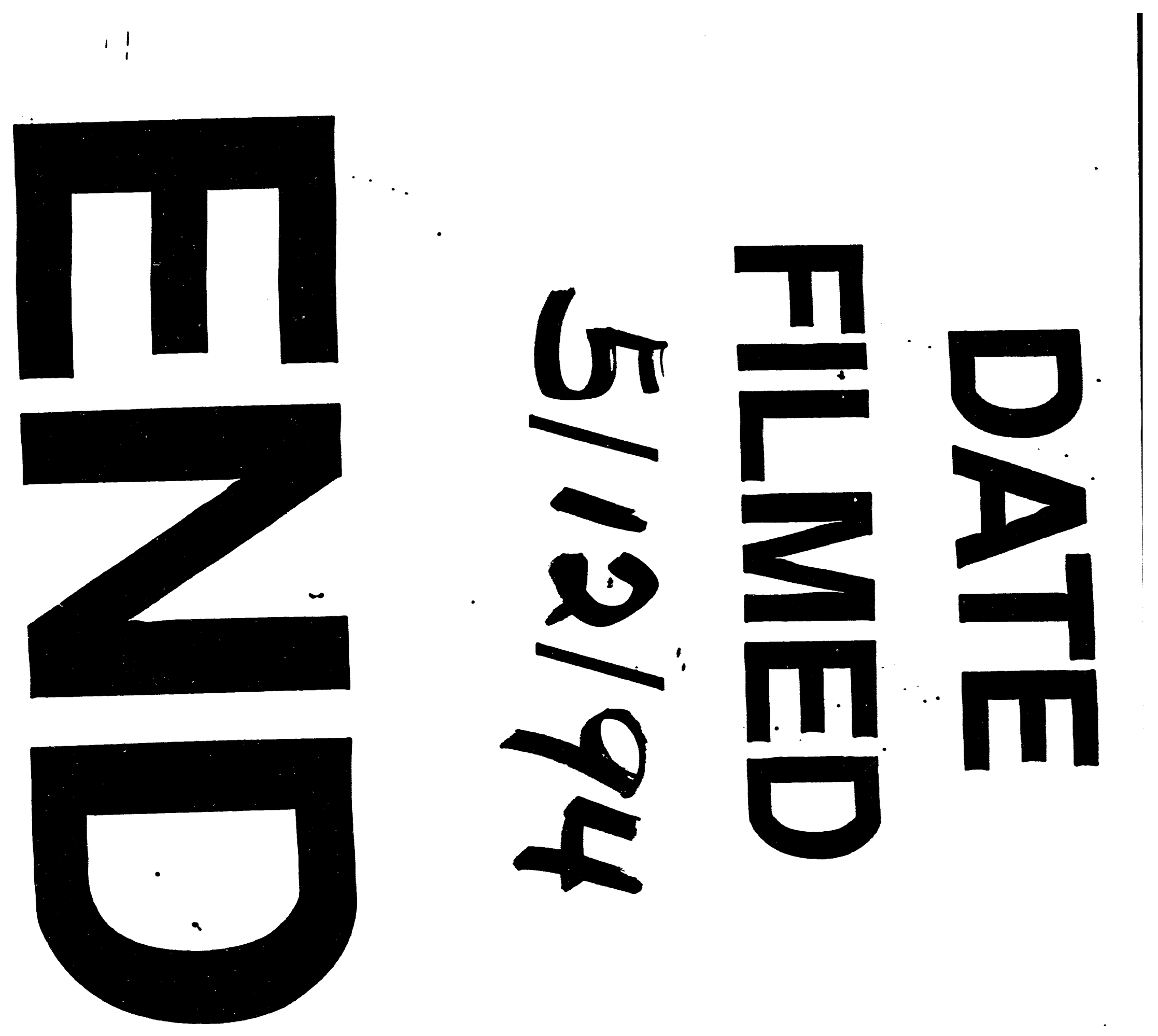
$$
-c_{-1}-1
$$

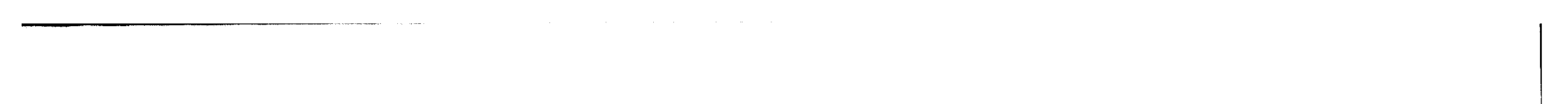

